\title{
A Metallaphotoredox Method for the Expansion of Benzyl SAR on Electron Deficient Amines
}

\author{
Meghan D. Shea, Umar Faruk Mansoor and Brett A. Hopkins
}

Discovery Chemistry, Merck Research Laboratories, 33 Avenue Louis Pasteur, Boston, MA 02115, United States

\section{Supporting Information}

Experimental procedures and characterization data for new compounds.

\section{Table of Contents}

General Considerations

Reaction Setup (General procedure A and B)

Synthesis of substrates

Flow Setup

Optimization attempts of $2 \mathrm{k}$

Other aryl halide SAR

General: As stated in general procedure A, all reactions were conducted in either the Penn photoreactor with a $450 \mathrm{~nm}$ light module (model number), or the hepatochem PhotoRedOX Duo photoreactor which was equipped with two 40 W Kessil A160WE lamps at maximum intensity. Unless otherwise stated all reagents and catalyst were from commercial sources and used as received. Anhydrous dimethylacetamide (DMA) was 
purchased from Sigma-Aldrich in a sure seal bottle and used as received. The $\left[\mathrm{Ni}(\mathrm{dtbbpy})(\mathrm{OH})_{4}\right] \mathrm{Cl}_{2}$ nickel precatalyst was made according to literature precedant. ${ }^{1}$ Yields refer to isolated compounds that are estimated to be $\geq 95 \%$ pure as judged by ${ }^{1} \mathrm{H}$ NMR or LC analysis. The yields reported in the supporting information describe the result of a single experiment, whereas yields reported in the paper are average yields of two or more experiments. Thus, the yields reported in the supporting information may slightly differ from those in the manuscript. Also, the NMR yields in the paper might be slightly lower than the isolated yields, as the reactions for NMR yields went through an aqueous workup before being dissolved in NMR solvent with an internal standard. Thus, some of the product could have been lost in the workup, whereas the isolated products were evaporated in vacuo and placed straight on the column for purification with no workup step. For NMR spectra, the chemical shifts in the spectra were referenced to the residual solvent peaks.

General Procedure A: arylation of amino acids: In air, the aryl bromide (1.0 equiv.), the appropriate $\mathrm{N}$-linked carboxylic acid (1.5 equiv.), $\left[\mathrm{Ni}(\mathrm{dtbbpy})\left(\mathrm{H}_{2} \mathrm{O}\right)_{4}\right] \mathrm{Cl}_{2}(10 \mathrm{~mol} \%)$, and $\operatorname{Ir}\left[\mathrm{dF}\left(\mathrm{CF}_{3}\right) \text { ppy }\right]_{2}(\mathrm{dtbbpy})\left(\mathrm{PF}_{6}\right)(1.0 \mathrm{~mol} \%)$ were added to a dry $8 \mathrm{~mL}$ teflon capped vial unless otherwise stated. DMA $(0.10 \mathrm{M})$ was then added to the vial, followed by DBU (1.5 equiv.). The vial was sparged with argon for two minutes, sealed, and placed in the PennOC/ Merck photoreactor equipped with a $450 \mathrm{~nm}$ blue LED light. The fan speed was set at approximately $2600 \mathrm{rpm}$, a stir rate of $1000 \mathrm{rpm}$, and an LED intensity of $100 \%$. If otherwise stated, the reaction could also be ran on the hepatochem PhotoRedOX Duo photoreactor which was equipped with two $40 \mathrm{~W}$ Kessil A160WE lamps at maximum intensity. The reactions were allowed to stir for 5-15 hours (see specific examples for exact time) and then the crude material was concentrated and purified via automated column chromatography to afford the desired product.

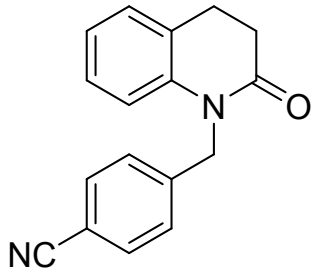

4-((2-oxo-3,4-dihydroquinolin-1(2H)-yl)methyl)benzonitrile (2a). General procedure 
A was employed for the reaction of 4-bromobenzonitrile $(46 \mathrm{mg}, 0.25 \mathrm{mmol})$ with 2-(2oxo-3,4-dihydroquinolin-1(2H)-yl)acetic acid (77 $\mathrm{mg}, \quad 0.375 \quad \mathrm{mmol})$. The [Ni(dtbbpy)(H2O)4]Cl2 (11.7 mg, $0.025 \mathrm{mmol})$ was used, which was added along with the $\operatorname{Ir}[\mathrm{dF}(\mathrm{CF} 3)$ ppy]2(dtbbpy)(PF6) $(2.5 \mathrm{mg}, 2.5 \mu \mathrm{mol})$. DMA (2.5 mL) and DBU (57 $\mu \mathrm{L}$, $0.375 \mathrm{mmol}$ ) were then added, and the reaction was stirred for 15 hours on hepatochem PhotoRedOX Duo photoreactor which was equipped with two 40 W Kessil A160WE lamps at maximum intensity. This procedure afforded the title compound (57.4 mg, 88\%), isolated via silica gel column chromatography (eluent hexanes and ethyl acetate) as a white solid: ${ }^{1} \mathrm{H}$ NMR (600 MHz, DMSO-d6) $\delta 7.77$ (d, J = 8.2 Hz, 2H), 7.42 (d, J = 8.2 Hz, 2H), $7.23(\mathrm{~d}, \mathrm{~J}=7.2 \mathrm{~Hz}, 1 \mathrm{H}), 7.11(\mathrm{t}, \mathrm{J}=7.8 \mathrm{~Hz}, 1 \mathrm{H}), 6.97(\mathrm{t}, \mathrm{J}=7.4 \mathrm{~Hz}, 1 \mathrm{H}), 6.84(\mathrm{~d}, \mathrm{~J}$ $=8.1 \mathrm{~Hz}, 1 \mathrm{H}), 5.23(\mathrm{~s}, 2 \mathrm{H}), 2.96(\mathrm{t}, \mathrm{J}=7.3 \mathrm{~Hz}, 2 \mathrm{H}), 2.71(\mathrm{t}, \mathrm{J}=7.4 \mathrm{~Hz}, 2 \mathrm{H}) .{ }^{13} \mathrm{C}$ NMR $(151$ $\mathrm{MHz}$, DMSO) $\delta 169.8,143.4,139.0,132.5,127.9,127.4,127.2,126.4,122.7,118.8$, 115.1, 109.7, 44.5, 31.1, 24.6. HRMS (ESI+) 263.1190 (263.1184 calcd for $\mathrm{C}_{17} \mathrm{H}_{14} \mathrm{~N}_{2} \mathrm{O}$, $\left.\mathrm{M}+\mathrm{H}^{+}\right)$.

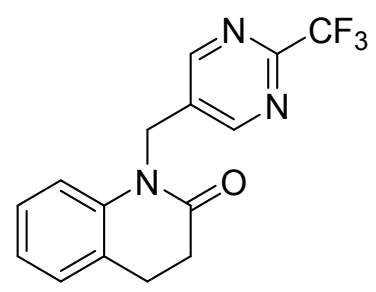

1-((2-(trifluoromethyl)pyrimidin-5-yl)methyl)-3,4-dihydroquinolin-2(1H)-on

(2b).

General procedure A was employed for the reaction of 5-bromo-2(trifluoromethyl)pyrimidine (57 mg, $0.25 \mathrm{mmol}$ ) with 2-(2-oxo-3,4-dihydroquinolin-1(2H)$\mathrm{yl})$ acetic acid $(77 \mathrm{mg}, 0.375 \mathrm{mmol})$. In this case, $5 \mathrm{~mol} \%$ [Ni(dtbbpy)(H2O)4]Cl2 (5.8 mg, $0.013 \mathrm{mmol}$ ) was used, which was added along with the $\operatorname{Ir}[\mathrm{dF}(\mathrm{CF} 3)$ ppy]2(dtbbpy)(PF6) (2.5 mg, $2.5 \mu \mathrm{mol})$. DMA ( $2.5 \mathrm{~mL})$ and DBU $(57 \mu \mathrm{L}, 0.375 \mathrm{mmol})$ were then added, and the reaction was stirred for 5 hours. This procedure afforded the title compound $(42.2 \mathrm{mg}$, $56 \%$ ), isolated via silica gel column chromatography (eluent hexanes and ethyl acetate) as a white solid: ${ }^{1} \mathrm{H}$ NMR (600 MHz, DMSO-d6) $\delta 8.95(\mathrm{~s}, 2 \mathrm{H}), 7.25(\mathrm{~d}, \mathrm{~J}=7.3 \mathrm{~Hz}, 1 \mathrm{H})$, $7.15(\mathrm{t}, \mathrm{J}=7.5 \mathrm{~Hz}, 1 \mathrm{H}), 7.03-6.97(\mathrm{~m}, 2 \mathrm{H}), 5.31(\mathrm{~s}, 2 \mathrm{H}), 2.98(\mathrm{t}, \mathrm{J}=7.1 \mathrm{~Hz}, 2 \mathrm{H}), 2.73(\mathrm{t}$, $\mathrm{J}=7.3 \mathrm{~Hz}, 2 \mathrm{H}$ ); ${ }^{13} \mathrm{C}$ NMR $\delta 170.2,157.0,153.9$ (q, $\left.\mathrm{J}=35.9 \mathrm{~Hz}\right), 138.8,134.2,128.0$, 127.4, 126.6, 122.9, 119.7 (q, J = 275.1 Hz), 115.0, 40.7, 31.0, 24.5. HRMS (ESI+) 308.1017 (308.1010 calcd for $\mathrm{C}_{15} \mathrm{H}_{12} \mathrm{~F}_{3} \mathrm{~N}_{3} \mathrm{O}, \mathrm{M}+\mathrm{H}^{+}$). 


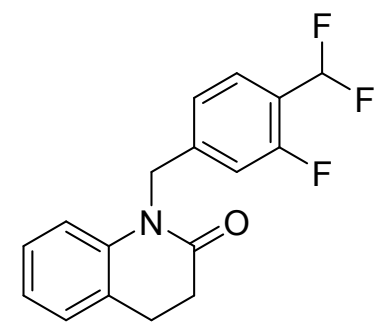

1-(4-(difluoromethyl)-3-fluorobenzyl)-3,4-dihydroquinolin-2(1H)-one (2c). General procedure A was employed for the reaction of 4-bromo-1-(difluoromethyl)-2fluorobenzene (56 mg, $0.25 \mathrm{mmol}$ ) with 2-(2-oxo-3,4-dihydroquinolin-1(2H)-yl)acetic acid $(77 \mathrm{mg}, 0.375 \mathrm{mmol})$. The $[\mathrm{Ni}(\mathrm{dtbbpy})(\mathrm{H} 2 \mathrm{O}) 4] \mathrm{Cl} 2(11.7 \mathrm{mg}, 0.025 \mathrm{mmol})$ was used, which was added along with the $\operatorname{Ir}[\mathrm{dF}(\mathrm{CF} 3)$ ppy]2(dtbbpy)(PF6) $(2.5 \mathrm{mg}, 2.5 \mu \mathrm{mol})$. DMA $(2.5 \mathrm{~mL})$ and DBU $(57 \mu \mathrm{L}, 0.375 \mathrm{mmol})$ were then added, and the reaction was stirred for 5 hours. This procedure afforded the title compound $(46.6 \mathrm{mg}, 61 \%)$, isolated via silica gel column chromatography (eluent hexanes and ethyl acetate) as a white solid: ${ }^{1} \mathbf{H}$ NMR (600 MHz, DMSO-d6) $\delta 7.58$ (t, J = 7.7 Hz, 1H), $7.25-7.19$ (m, 3H), 7.14 (dd, J = 14.7, $6.7 \mathrm{~Hz}, 1 \mathrm{H}), 6.97(\mathrm{t}, \mathrm{J}=7.4 \mathrm{~Hz}, 1 \mathrm{H}), 6.88(\mathrm{~d}, \mathrm{~J}=8.1 \mathrm{~Hz}, 1 \mathrm{H}), 5.21(\mathrm{~s}, 2 \mathrm{H}), 3.36(\mathrm{~d}, \mathrm{~J}=$ $1.4 \mathrm{~Hz}, 1 \mathrm{H}), 2.96(\mathrm{t}, \mathrm{J}=7.5 \mathrm{~Hz}, 2 \mathrm{H}), 2.72(\mathrm{t}, \mathrm{J}=7.1,2 \mathrm{H}) ;{ }^{13} \mathrm{C}$ NMR $\delta 169.9,159.9$ (dt, J $=250.7,5.0 \mathrm{~Hz}), 143.9(\mathrm{~d}, \mathrm{~J}=7.5 \mathrm{~Hz}), 139.0,127.9,127.7(\mathrm{~d}, \mathrm{~J}=3.0 \mathrm{~Hz}), 127.2,126.4$, 122.7, 122.6 (d, J = 2.9 Hz), 119.8 (td, J = 23.1, $12.5 \mathrm{~Hz}), 115.1,114.2$ (d, J = 21.0 Hz), 112.0 (td, $\mathrm{J}=235.4,3.6 \mathrm{~Hz}$ ), 44.2, 31.1, 24.6. HRMS (ESI+) 306.1120 (306.1105 calcd for $\mathrm{C}_{17} \mathrm{H}_{14} \mathrm{~F}_{3} \mathrm{NO}, \mathrm{M}+\mathrm{H}^{+}$).

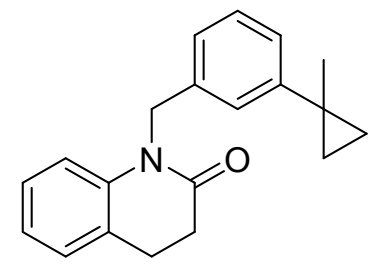

1-(3-(1-methylcyclopropyl)benzyl)-3,4-dihydroquinolin-2(1H)-one (2d). General procedure A was employed for the reaction of 1-bromo-3-(1-methylcyclopropyl)benzene (42 mg, $0.20 \mathrm{mmol}$ ) with 2-(2-oxo-3,4-dihydroquinolin-1(2H)-yl)acetic acid (62 mg, 0.30 $\mathrm{mmol})$. The $[\mathrm{Ni}(\mathrm{dtbbpy})(\mathrm{H} 2 \mathrm{O}) 4] \mathrm{Cl} 2(11.7 \mathrm{mg}, 0.020 \mathrm{mmol})$ was used, which was added along with the $\operatorname{Ir}[\mathrm{dF}(\mathrm{CF} 3)$ ppy]2(dtbbpy)(PF6) $(2.0 \mathrm{mg}, 2.0 \mu \mathrm{mol})$. DMA $(2.0 \mathrm{~mL})$ and DBU $(45 \mu \mathrm{L}, 0.30 \mathrm{mmol})$ were then added, and the reaction was stirred for 5 hours. This 
procedure afforded the title compound (37.5 mg, 64\%), isolated via silica gel column chromatography (eluent hexanes and ethyl acetate) as an oil: ${ }^{1} \mathrm{H}$ NMR $(600 \mathrm{MHz}$, DMSOd6) $\delta 7.22$ (d, J = 7.3 Hz, 1H), $7.17(\mathrm{t}, \mathrm{J}=7.7 \mathrm{~Hz}, 1 \mathrm{H}), 7.13-7.09(\mathrm{~m}, 2 \mathrm{H}), 7.05$ (d, J = $7.7 \mathrm{~Hz}, 1 \mathrm{H}), 6.96-6.91(\mathrm{~m}, 3 \mathrm{H}), 5.11(\mathrm{~s}, 2 \mathrm{H}), 2.92(\mathrm{t}, \mathrm{J}=7.6,2 \mathrm{H}), 2.68(\mathrm{t}, \mathrm{J}=7.4,2 \mathrm{H})$, $1.33(\mathrm{~s}, 3 \mathrm{H}), 0.80-0.70(\mathrm{~m}, 4 \mathrm{H}) .{ }^{13} \mathrm{C}$ NMR $(151 \mathrm{MHz}$, DMSO) $\delta 169.7,146.7,139.3$, $137.1,128.3,127.8,127.2,126.4,124.4,124.1,123.1,122.5,115.4,44.5,31.3,24.8$, 24.7, 18.9, 16.0. HRMS (ESI+) 292.1720 (292.1701 calcd for $\left.\mathrm{C}_{20} \mathrm{H}_{21} \mathrm{NO}, \mathrm{M}+\mathrm{H}^{+}\right)$.

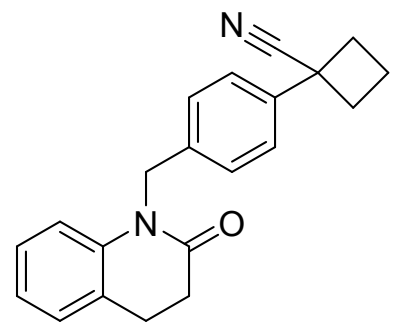

1-(4-((2-oxo-3,4-dihydroquinolin-1(2H)-yl)methyl)phenyl)cyclobutane-1-

carbonitrile (2e). General procedure A was employed for the reaction of 1-(4bromophenyl)cyclobutane-1-carbonitrile $(59 \mathrm{mg}, \quad 0.25 \mathrm{mmol})$ with 2-(2-oxo-3,4dihydroquinolin-1(2H)-yl)acetic acid $(77 \mathrm{mg}, 0.375 \mathrm{mmol})$. The [Ni(dtbbpy)(H2O)4]Cl2 $(11.7 \mathrm{mg}, \quad 0.025 \mathrm{mmol})$ was used, which was added along with the Ir[dF(CF3)ppy]2(dtbbpy)(PF6) (2.5 mg, $2.5 \mu \mathrm{mol})$. DMA (2.5 mL) and DBU (57 $\mu \mathrm{L}, 0.375$ $\mathrm{mmol}$ ) were then added, and the reaction was stirred for 5 hours. This procedure afforded the title compound ( $38.8 \mathrm{mg}, 49 \%$ ), isolated via silica gel column chromatography (eluent hexanes and ethyl acetate) as an oil: ${ }^{1} \mathrm{H}$ NMR (600 MHz, DMSO-d6) $\delta 7.39$ (d, J = 8.2 $\mathrm{Hz}, 2 \mathrm{H}), 7.29$ (d, J = 8.2 Hz, 2H), $7.22(\mathrm{~d}, \mathrm{~J}=7.3 \mathrm{~Hz}, 1 \mathrm{H}), 7.13(\mathrm{t}, \mathrm{J}=7.6 \mathrm{~Hz}, 1 \mathrm{H}), 6.98-$ $6.91(\mathrm{~m}, 2 \mathrm{H}), 5.16(\mathrm{~s}, 2 \mathrm{H}), 2.94(\mathrm{t}, \mathrm{J}=7.3 \mathrm{~Hz}, 2 \mathrm{H}), 2.72-2.66(\mathrm{~m}, 4 \mathrm{H}), 2.61-2.53(\mathrm{~m}$, $2 \mathrm{H}), 2.29-2.20(\mathrm{~m}, 1 \mathrm{H}), 2.01-1.93(\mathrm{~m}, 1 \mathrm{H}) .{ }^{13} \mathrm{C}$ NMR (151 MHz, DMSO) $\delta 169.7,139.2$, 138.2, 137.1, 127.9, 127.2, 127.0, 126.4, 125.8, 124.3, 122.6, 115.2, 44.1, 33.8, 31.2, 24.7, 16.7. HRMS (ESI+) $317.1663\left(317.1654\right.$ calcd for $\left.\mathrm{C}_{21} \mathrm{H}_{20} \mathrm{~N}_{2} \mathrm{O}, \mathrm{M}+\mathrm{H}^{+}\right)$.

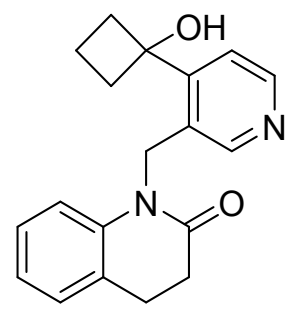

1-((4-(1-hydroxycyclobutyl)pyridin-3-yl)methyl)-3,4-dihydroquinolin-2(1H)-one (2f). 
General procedure A was employed for the reaction of 1-(3-bromopyridin-4-yl)cyclobutan1-ol (57 mg, $0.25 \mathrm{mmol})$ with 2-(2-oxo-3,4-dihydroquinolin-1(2H)-yl)acetic acid (77 mg, $0.375 \mathrm{mmol})$. The $[\mathrm{Ni}(\mathrm{dtbbpy})(\mathrm{H} 2 \mathrm{O}) 4] \mathrm{Cl} 2(11.7 \mathrm{mg}, 0.025 \mathrm{mmol})$ was used, which was added along with the $\operatorname{Ir}[\mathrm{dF}(\mathrm{CF} 3) \mathrm{ppy}] 2$ (dtbbpy)(PF6) (2.5 mg, $2.5 \mu \mathrm{mol})$. DMA (2.5 mL) and DBU $(57 \mu \mathrm{L}, 0.375 \mathrm{mmol})$ were then added, and the reaction was stirred for 5 hours. This procedure afforded the title compound $(48.9 \mathrm{mg}, 63 \%)$, isolated via silica gel column chromatography (eluent hexanes and ethyl acetate) as a tan solid: ${ }^{1} \mathbf{H} \mathbf{N M R}(600 \mathrm{MHz}$, DMSO-d6) $\delta 8.44(\mathrm{~d}, \mathrm{~J}=4.9 \mathrm{~Hz}, 1 \mathrm{H}$ ), $8.00(\mathrm{~s}, 1 \mathrm{H}), 7.37$ (d, J = $5.0 \mathrm{~Hz}, 1 \mathrm{H}), 7.26$ (d, J = $7.2 \mathrm{~Hz}, 1 \mathrm{H}), 7.09$ (t, J = 7.3 Hz, 1H), $6.97(\mathrm{t}, \mathrm{J}=7.4 \mathrm{~Hz}, 1 \mathrm{H}), 6.78(\mathrm{~d}, \mathrm{~J}=8.1 \mathrm{~Hz}, 1 \mathrm{H})$, $5.96(\mathrm{~s}, 1 \mathrm{H}), 5.28(\mathrm{~s}, 2 \mathrm{H}), 3.00(\mathrm{t}, \mathrm{J}=7.5 \mathrm{~Hz}, 2 \mathrm{H}), 2.75(\mathrm{t}, \mathrm{J}=7.3 \mathrm{~Hz}, 2 \mathrm{H}), 2.66-2.60(\mathrm{~m}$, $2 \mathrm{H}), 2.38-2.31(\mathrm{~m}, 2 \mathrm{H}), 2.07-1.98(\mathrm{~m}, 1 \mathrm{H}), 1.66-1.58(\mathrm{~m}, 1 \mathrm{H}) .{ }^{13} \mathrm{C}$ NMR $(151 \mathrm{MHz}$, DMSO) $\delta 169.9,150.6,148.4,146.5,139.3,130.1,127.9,127.3,126.3,122.6,119.7$, 115.1, 75.8, 41.8, 35.0, 31.1, 24.7, 13.5. HRMS (ESI+) 309.1615 (309.1603 calcd for $\mathrm{C}_{19} \mathrm{H}_{20} \mathrm{~N}_{2} \mathrm{O}_{2}, \mathrm{M}+\mathrm{H}^{+}$).

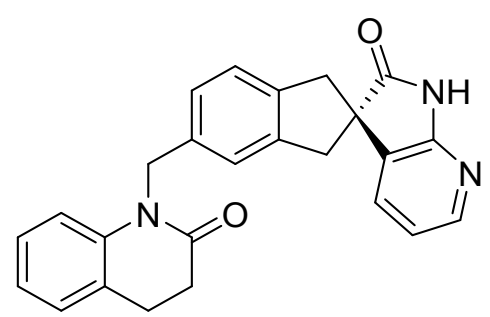

(S)-5-((2-oxo-3,4-dihydroquinolin-1(2H)-yl)methyl)-1,3-dihydrospiro[indene-2,3'pyrrolo[2,3-b]pyridin]-2'(1'H)-one (2g). General procedure A was employed for the reaction of (S)-5-bromo-1,3-dihydrospiro[indene-2,3'-pyrrole[2,3-b]pyridine]-2'(1'H)-one (79 mg, $0.25 \mathrm{mmol}$ ) with 2-(2-oxo-3,4-dihydroquinolin-1(2H)-yl)acetic acid (77 mg, 0.375 $\mathrm{mmol})$. The $[\mathrm{Ni}(\mathrm{dtbbpy})(\mathrm{H} 2 \mathrm{O}) 4] \mathrm{Cl} 2(11.7 \mathrm{mg}, 0.025 \mathrm{mmol})$ was used, which was added along with the $\operatorname{Ir}[\mathrm{dF}(\mathrm{CF} 3)$ ppy]2(dtbbpy)(PF6) $(2.5 \mathrm{mg}, 2.5 \mu \mathrm{mol})$. DMA (2.5 mL) and DBU $(57 \mu \mathrm{L}, 0.375 \mathrm{mmol})$ were then added, and the reaction was stirred for 12 hours. This procedure afforded the title compound $(57.5 \mathrm{mg}, 58 \%)$, isolated via silica gel column chromatography (eluent hexanes and ethyl acetate) as a tan solid: ${ }^{1} \mathrm{H}$ NMR $(600 \mathrm{MHz}$, DMSO-d6) $\delta 11.05$ (s, 1H), 8.05 (dd, J = 5.2, $1.5 \mathrm{~Hz}, 1 \mathrm{H}$ ), $7.24-7.19(\mathrm{~m}, 3 \mathrm{H}), 7.16-$ $7.12(\mathrm{~m}, 2 \mathrm{H}), 7.10(\mathrm{~d}, \mathrm{~J}=7.8 \mathrm{~Hz}, 1 \mathrm{H}), 6.96(\mathrm{t}, \mathrm{J}=7.7 \mathrm{~Hz}, 2 \mathrm{H}), 6.86(\mathrm{dd}, \mathrm{J}=7.3,5.3 \mathrm{~Hz}$, $1 \mathrm{H}), 5.14(\mathrm{~s}, 2 \mathrm{H}), 3.30(\mathrm{~d}, \mathrm{~J}=16.1 \mathrm{~Hz}, 2 \mathrm{H}), 3.09(\mathrm{~d}, \mathrm{~J}=15.9 \mathrm{~Hz}, 2 \mathrm{H}), 2.94(\mathrm{t}, \mathrm{J}=7.4 \mathrm{~Hz}$, 
2H), $2.70(\mathrm{t}, \mathrm{J}=7.4 \mathrm{~Hz}, 2 \mathrm{H}) .{ }^{13} \mathrm{C}$ NMR (151 MHz, DMSO) $\delta$ 180.7, 169.7, 156.2, 146.6, $141.5,139.7,139.4,136.2,129.6,129.4,127.8,127.2,126.4,125.2,124.4,122.5,122.3$, 117.8, 115.4, 53.5, 44.6, 42.8, 42.5, 31.2, 24.7. HRMS (ESI+) 396.1724 (396.1712 calcd for $\mathrm{C}_{25} \mathrm{H}_{21} \mathrm{~N}_{3} \mathrm{O}_{2}, \mathrm{M}+\mathrm{H}^{+}$).

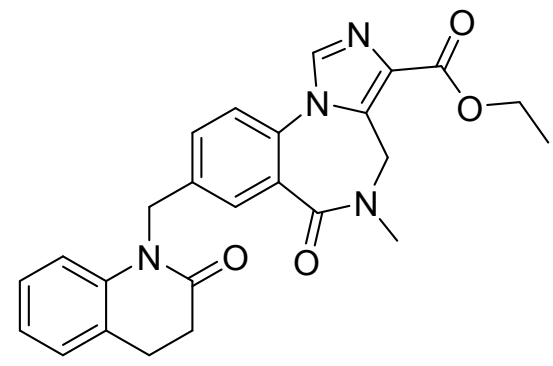

ethyl 5-methyl-6-oxo-8-((2-oxo-3,4-dihydroquinolin-1(2H)-yl)methyl)-5,6-dihydro4H-benzo[f]imidazo[1,5-a][1,4]diazepine-3-carboxylate $(2 \mathrm{~h})$. General procedure A was employed for the reaction of ethyl 8-bromo-5-methyl-6-oxo-5,6-dihydro-4Hbenzo[f]imidazo[1,5-a][1,4]diazepine-3-carboxylate $(55 \mathrm{mg}, 0.15 \mathrm{mmol})$ with 2-(2-oxo3,4-dihydroquinolin-1(2H)-yl)acetic acid (46 mg, $0.225 \mathrm{mmol})$. The [Ni(dtbbpy)(H2O)4]Cl2 $(7.0 \mathrm{mg}, \quad 0.015 \mathrm{mmol})$ was used, which was added along with the $\operatorname{Ir}[\mathrm{dF}(\mathrm{CF} 3)$ ppy]2(dtbbpy)(PF6) (1.5 mg, $1.5 \mu \mathrm{mol})$. DMA (1.5 mL) and DBU (34 $\mu \mathrm{L}, 0.225$ $\mathrm{mmol}$ ) were then added, and the reaction was stirred for 5 hours. This procedure afforded the title compound $(51.0 \mathrm{mg}, 76 \%$ ), isolated via silica gel column chromatography (eluent hexanes and ethyl acetate) as a tan solid: ${ }^{1} \mathrm{H}$ NMR $(600 \mathrm{MHz}, \mathrm{DMSO}-\mathrm{d} 6) \delta 8.34(\mathrm{~s}, 1 \mathrm{H})$, $7.79(\mathrm{~d}, \mathrm{~J}=1.7 \mathrm{~Hz}, 1 \mathrm{H}), 7.67(\mathrm{~d}, \mathrm{~J}=8.3 \mathrm{~Hz}, 1 \mathrm{H}), 7.56(\mathrm{dd}, \mathrm{J}=8.3,1.8 \mathrm{~Hz}, 1 \mathrm{H}), 7.25$ (d, $\mathrm{J}=7.2 \mathrm{~Hz}, 1 \mathrm{H}), 7.15(\mathrm{t}, \mathrm{J}=7.3 \mathrm{~Hz}, 1 \mathrm{H}), 7.01-6.95(\mathrm{~m}, 2 \mathrm{H}), 5.26(\mathrm{~s}, 2 \mathrm{H}), 5.03-4.39(\mathrm{~m}$, 2H), $4.31(\mathrm{~s}, 2 \mathrm{H}), 3.08(\mathrm{~s}, 3 \mathrm{H}), 2.95(\mathrm{t}, \mathrm{J}=7.3 \mathrm{~Hz}, 2 \mathrm{H}), 2.72(\mathrm{t}, \mathrm{J}=7.3 \mathrm{~Hz}, 2 \mathrm{H}), 1.32(\mathrm{t}, \mathrm{J}$ $=7.1 \mathrm{~Hz}, 3 \mathrm{H}) .{ }^{13} \mathrm{C}$ NMR $(151 \mathrm{MHz}$, DMSO) $\delta 169.9,165.5,162.3,139.1,137.7,136.4$, 135.4, 130.7, 130.6, 129.5, 128.6, 128.0, 127.5, 127.3, 126.4, 123.2, 122.8, 115.2, 60.09, 44.0, 42.0, 35.1, 31.2, 24.7, 14.2. HRMS (ESI+) 445.1888 (445.1876 calcd for $\left.\mathrm{C}_{25} \mathrm{H}_{24} \mathrm{~N}_{4} \mathrm{O}_{4}, \mathrm{M}+\mathrm{H}^{+}\right)$.<smiles>N#Cc1ccc(CN2C(=O)CSc3ccccc32)cc1</smiles> 
4-((3-oxo-2,3-dihydro-4H-benzo[b][1,4]thiazin-4-yl)methyl)benzonitrile (2i). General procedure A was employed for the reaction of 4-bromobenzonitrile $(46 \mathrm{mg}, 0.25 \mathrm{mmol})$ with 2-(3-oxo-2,3-dihydro-4H-benzo[b][1,4]thiazin-4-yl)acetic acid (84 mg, $0.375 \mathrm{mmol}$ ). The $[\mathrm{Ni}(\mathrm{dtbbpy})(\mathrm{H} 2 \mathrm{O}) 4] \mathrm{Cl} 2(11.7 \mathrm{mg}, 0.025 \mathrm{mmol})$ was used, which was added along with the $\operatorname{Ir}[\mathrm{dF}(\mathrm{CF} 3)$ ppy]2(dtbbpy)(PF6) $(2.5 \mathrm{mg}, 2.5 \mu \mathrm{mol})$. DMA $(2.5 \mathrm{~mL})$ and DBU (57 $\mu \mathrm{L}, 0.375 \mathrm{mmol}$ ) were then added, and the reaction was stirred for 15 hours on the hepatochem PhotoRedOX Duo photoreactor which was equipped with two $40 \mathrm{~W}$ Kessil A160WE lamps at maximum intensity. This procedure afforded the title compound (61.7 $\mathrm{mg}, 88 \%$ ), isolated via silica gel column chromatography (eluent hexanes and ethyl acetate) as an oil: ${ }^{1} \mathrm{H}$ NMR (600 MHz, DMSO-d6) $\delta 7.79(\mathrm{~d}, \mathrm{~J}=8.3 \mathrm{~Hz}, 2 \mathrm{H}), 7.43$ (dd, J = 7.7, $1.4 \mathrm{~Hz}, 1 \mathrm{H}), 7.39(\mathrm{~d}, \mathrm{~J}=8.3 \mathrm{~Hz}, 2 \mathrm{H}), 7.20-7.16(\mathrm{~m}, 1 \mathrm{H}), 7.08(\mathrm{~d}, \mathrm{~J}=7.7 \mathrm{~Hz}, 1 \mathrm{H})$, $7.04-7.00(\mathrm{~m}, 1 \mathrm{H}), 5.31$ (s, 2H), 3.69 (s, 2H). ${ }^{13} \mathrm{C}$ NMR (151 MHz, DMSO) $\delta$ 165.4, 143.0, 138.9, 132.5, 128.2, 127.3, 127.2, 123.6, 123.2, 118.7, 118.1, 109.8, 46.6, 30.2. HRMS (ESI+) $281.0750\left(281.0748\right.$ calcd for $\left.\mathrm{C}_{16} \mathrm{H}_{12} \mathrm{~N}_{2} \mathrm{OS}, \mathrm{M}+\mathrm{H}^{+}\right)$.

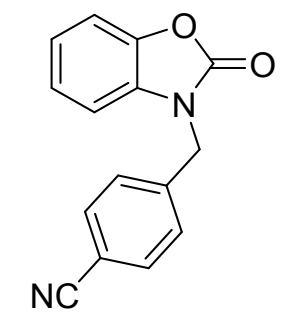

4-((2-oxobenzo[d]oxazol-3(2H)-yl)methyl)benzonitrile (2j). General procedure A was employed for the reaction of 4-bromobenzonitrile $(46 \mathrm{mg}, 0.25 \mathrm{mmol}$ ) with 2-(2oxobenzo[d]oxazol-3(2H)-yl)acetic acid ( $72 \mathrm{mg}, 0.375 \mathrm{mmol})$. The [Ni(dtbbpy)(H2O)4]Cl2 $(11.7 \mathrm{mg}, \quad 0.025 \mathrm{mmol})$ was used, which was added along with the Ir[dF(CF3)ppy]2(dtbbpy)(PF6) (2.5 mg, $2.5 \mu \mathrm{mol})$. DMA (2.5 mL) and DBU (57 $\mu \mathrm{L}, 0.375$ $\mathrm{mmol}$ ) were then added, and the reaction was stirred for 15 hours on the hepatochem PhotoRedOX Duo photoreactor which was equipped with two 40 W Kessil A160WE lamps at maximum intensity. This procedure afforded the title compound (32.9 $\mathrm{mg}, 53 \%$ ), isolated via silica gel column chromatography (eluent hexanes and ethyl acetate) as a white solid: ${ }^{1} \mathrm{H}$ NMR $(600 \mathrm{MHz}$, DMSO-d6) $\delta 7.84$ (d, J = 8.2 Hz, 2H), 7.56 (d, J = $8.2 \mathrm{~Hz}$, 2H), $7.38(\mathrm{~d}, \mathrm{~J}=7.7 \mathrm{~Hz}, 1 \mathrm{H}), 7.22-7.12(\mathrm{~m}, 3 \mathrm{H}), 5.16(\mathrm{~s}, 2 \mathrm{H}) \cdot{ }^{13} \mathrm{C}$ NMR $(151 \mathrm{MHz}$, DMSO) $\delta 153.9,142.0,141.2,132.7,130.6,128.3,124.0,122.6,118.6,110.7,109.9$, 
109.4, 44.6. HRMS (ESI+) $251.0813\left(251.0820\right.$ calcd for $\left.\mathrm{C}_{15} \mathrm{H}_{10} \mathrm{~N}_{2} \mathrm{O}_{2}, \mathrm{M}+\mathrm{H}^{+}\right)$.

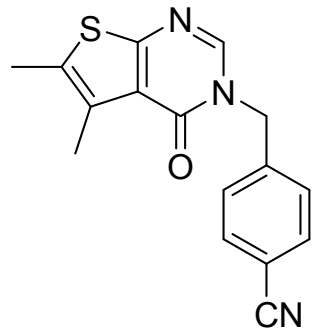

\section{4-((5,6-dimethyl-4-oxothieno[2,3-d]pyrimidin-3(4H)-yl)methyl)benzonitrile}

(2k).

General procedure A was employed for the reaction of 4-bromobenzonitrile ( $46 \mathrm{mg}, 0.25$ mmol) with 2-(5,6-dimethyl-4-oxothieno[2,3-d]pyrimidin-3(4H)-yl)acetic acid (89 mg, $0.375 \mathrm{mmol})$. The $[\mathrm{Ni}(\mathrm{dtbbpy})(\mathrm{H} 2 \mathrm{O}) 4] \mathrm{Cl} 2(11.7 \mathrm{mg}, 0.025 \mathrm{mmol})$ was used, which was added along with the $\operatorname{Ir}[\mathrm{dF}(\mathrm{CF} 3)$ ppy]2(dtbbpy)(PF6) $(2.5 \mathrm{mg}, 2.5 \mu \mathrm{mol})$. DMA (2.5 mL) and DBU ( $57 \mu \mathrm{L}, 0.375 \mathrm{mmol}$ ) were then added, and the reaction was stirred for 15 hours. This procedure afforded the title compound ( $17.3 \mathrm{mg}, 23 \%)$, isolated via silica gel column chromatography (eluent hexanes and ethyl acetate) as a light yellow solid: ${ }^{1} \mathbf{H}$ NMR (600 MHz, DMSO-d6) ס 8.54 (s, 1H), 7.82 (d, J = $8.3 \mathrm{~Hz}, 2 \mathrm{H}$ ), 7.50 (d, J = $8.3 \mathrm{~Hz}, 2 \mathrm{H}$ ), 5.24 (s,

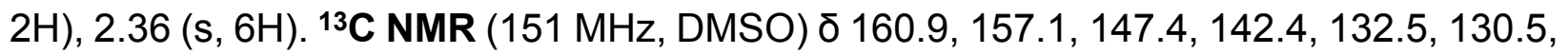
128.8, 128.4, 122.7, 118.6, 110.3, 48.4, 12.9, 12.7 HRMS (ESI+) 296.0862 (296.0857 calcd for $\mathrm{C}_{16} \mathrm{H}_{13} \mathrm{~N}_{3} \mathrm{OS}, \mathrm{M}+\mathrm{H}^{+}$).

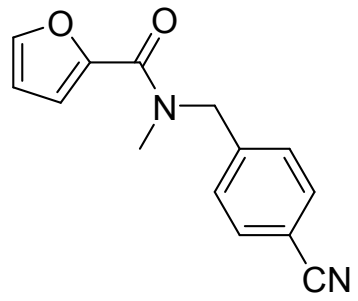

$\mathrm{N}$-(4-cyanobenzyl)-N-methylfuran-2-carboxamide (2I). General procedure A was employed for the reaction of 4-bromobenzonitrile (46 mg, $0.25 \mathrm{mmol}$ ) with $\mathrm{N}$-(furan-2carbonyl)-N-methylglycine (68 mg, $0.375 \mathrm{mmol})$. The $[\mathrm{Ni}(\mathrm{dtbbpy})(\mathrm{H} 2 \mathrm{O}) 4] \mathrm{Cl} 2(11.7 \mathrm{mg}$, $0.025 \mathrm{mmol}$ ) was used, which was added along with the $\operatorname{Ir}[\mathrm{dF}(\mathrm{CF} 3)$ ppy]2(dtbbpy)(PF6) (2.5 mg, $2.5 \mu \mathrm{mol})$. DMA (2.5 mL) and DBU $(57 \mu \mathrm{L}, 0.375 \mathrm{mmol})$ were then added, and the reaction was stirred for 15 hours on the hepatochem PhotoRedOX Duo photoreactor which was equipped with two $40 \mathrm{~W}$ Kessil A160WE lamps at maximum intensity. This procedure afforded the title compound (39 $\mathrm{mg}, 65 \%$ ), isolated via silica gel column chromatography (eluent hexanes and ethyl acetate) as a white solid: $1 \mathrm{H} \mathrm{NMR} \mathrm{(600} \mathrm{MHz,}$ 
DMSO-d6) ס 7.95-7.79 (m, 3H), 7.50 (d, J = 7.8 Hz, 2H), 7.20-6.88 (br m, 1H), 6.67 (br $\mathrm{s}, 1 \mathrm{H}), 5.01-4.67(\mathrm{~m}, 2 \mathrm{H}), 3.34-2.80(\mathrm{~m}, 3 \mathrm{H}) .{ }^{13} \mathrm{C}$ NMR (151 MHz, DMSO) $\delta 159.6,146.9$, $145.1,143.4,132.5,128.3,118.7,116.3,111.4,110.0,53.0,50.9,36.4,34.3$ (extra peaks in NMR due to rotamers). HRMS (ESI+) 241.0980 (241.0977 calcd for $\mathrm{C}_{14} \mathrm{H}_{12} \mathrm{~N}_{2} \mathrm{O}_{2}, \mathrm{M}+$ $\left.\mathrm{H}^{+}\right)$.

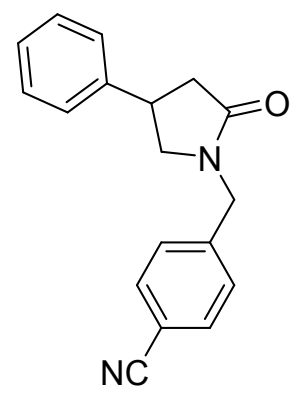

4-((2-oxo-4-phenylpyrrolidin-1-yl)methyl)benzonitrile (2m). General procedure A was employed for the reaction of 4-bromobenzonitrile ( $46 \mathrm{mg}, 0.25 \mathrm{mmol})$ with 2-(2-oxo-4phenylpyrrolidin-1-yl)acetic acid $(82 \mathrm{mg}, 0.375 \mathrm{mmol})$. The [Ni(dtbbpy) $(\mathrm{H} 2 \mathrm{O}) 4] \mathrm{Cl} 2(11.7$ $\mathrm{mg}, \quad 0.025 \mathrm{mmol}$ ) was used, which was added along with the $\operatorname{Ir}[\mathrm{dF}(\mathrm{CF} 3)$ ppy]2(dtbbpy)(PF6) (2.5 mg, $2.5 \mu \mathrm{mol})$. DMA (2.5 mL) and DBU (57 $\mu \mathrm{L}, 0.375$ $\mathrm{mmol}$ ) were then added, and the reaction was stirred for 15 hours on the hepatochem PhotoRedOX Duo photoreactor which was equipped with two 40 W Kessil A160WE lamps at maximum intensity. This procedure afforded the title compound (53.8 $\mathrm{mg}, 78 \%$ ), isolated via silica gel column chromatography (eluent hexanes and ethyl acetate) as an oil: ${ }^{1} \mathrm{H}$ NMR (600 MHz, DMSO-d6) $\delta 7.82(\mathrm{~d}, \mathrm{~J}=8.2 \mathrm{~Hz}, 2 \mathrm{H}), 7.46(\mathrm{~d}, \mathrm{~J}=8.2 \mathrm{~Hz}, 2 \mathrm{H})$, $7.35-7.20(\mathrm{~m}, 5 \mathrm{H}), 4.58-4.47(\mathrm{~m}, 2 \mathrm{H}), 3.63(\mathrm{dq}, \mathrm{J}=23.9,8.3 \mathrm{~Hz}, 2 \mathrm{H}), 3.24(\mathrm{dd}, \mathrm{J}=$ 8.8, $7.0 \mathrm{~Hz}, 1 \mathrm{H}), 2.76(\mathrm{dd}, \mathrm{J}=16.5,8.6 \mathrm{~Hz}, 1 \mathrm{H}), 2.55-2.51(\mathrm{~m}, 1 \mathrm{H}) .{ }^{13} \mathrm{C}$ NMR $(151 \mathrm{MHz}$, DMSO) $\delta 173.3,142.9,142.5,132.5,128.6,128.5,126.9,126.7,118.8,110.1,53.4,45.2$, 37.9, 36.8. HRMS (ESI+) $277.1349\left(277.1341\right.$ calcd for $\left.\mathrm{C}_{18} \mathrm{H}_{16} \mathrm{~N}_{2} \mathrm{O}, \mathrm{M}+\mathrm{H}^{+}\right)$.

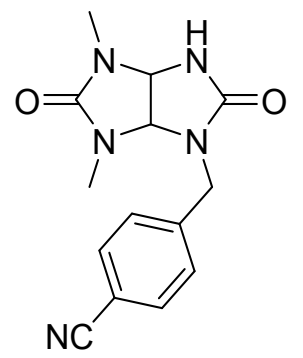




\section{4-((4,6-dimethyl-2,5-dioxohexahydroimidazo[4,5-d]imidazol-1(2H)-}

yl)methyl)benzonitrile (2n). General procedure A was employed for the reaction of 4bromobenzonitrile $(57 \quad \mathrm{mg}, \quad 0.25 \mathrm{mmol})$ with 2-(4,6-dimethyl-2,5dioxohexahydroimidazo[4,5-d]imidazol-1(2H)-yl)acetic acid (85 mg, $0.375 \mathrm{mmol}$ ). The [Ni(dtbbpy)(H2O)4]Cl2 (11.7 mg, $0.025 \mathrm{mmol})$ was used, which was added along with the $\operatorname{Ir}[\mathrm{dF}(\mathrm{CF} 3)$ ppy]2(dtbbpy)(PF6) $(2.5 \mathrm{mg}, 2.5 \mu \mathrm{mol})$. DMA (2.5 mL) and DBU (57 $\mu \mathrm{L}$, $0.375 \mathrm{mmol}$ ) were then added, and the reaction was stirred for 15 hours on the hepatochem PhotoRedOX Duo photoreactor which was equipped with two $40 \mathrm{~W}$ Kessil A160WE lamps at maximum intensity. This procedure afforded the title compound (40.9 $\mathrm{mg}, 57 \%$ ), isolated via mass directed reverse phase column chromatography with $0.1 \%$ TFA in Acetonitrile/Water; isolated as a white solid: ${ }^{1} \mathrm{H}$ NMR $(600 \mathrm{MHz}$, DMSO-d6) $\delta 7.92$ (s, 1H), $7.82(\mathrm{~d}, \mathrm{~J}=8.2 \mathrm{~Hz}, 2 \mathrm{H}), 7.44(\mathrm{~d}, \mathrm{~J}=8.2 \mathrm{~Hz}, 2 \mathrm{H}), 5.17(\mathrm{dd}, \mathrm{J}=8.3,1.6 \mathrm{~Hz}, 1 \mathrm{H})$, $5.11(\mathrm{~d}, \mathrm{~J}=8.3 \mathrm{~Hz}, 1 \mathrm{H}), 4.55-4.48(\mathrm{~m}, 2 \mathrm{H}), 2.67(\mathrm{~s}, 3 \mathrm{H}), 2.51(\mathrm{~s}, 3 \mathrm{H}) \cdot{ }^{13} \mathrm{C}$ NMR $(151$ $\mathrm{MHz}$, DMSO) $\delta 159.6,158.1,144.4,132.5,127.9,118.8,109.9,72.2$, 65.8, 45.5, 29.9, 27.9. HRMS (ESI+) $286.1314\left(286.1304\right.$ calcd for $\left.\mathrm{C}_{14} \mathrm{H}_{15} \mathrm{~N}_{5} \mathrm{O}_{2}, \mathrm{M}+\mathrm{H}^{+}\right)$.

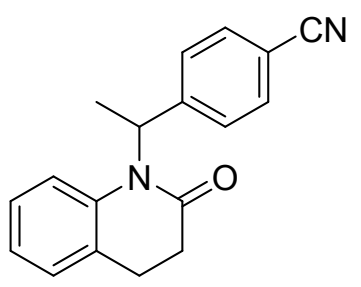

4-(1-(2-oxo-3,4-dihydroquinolin-1(2H)-yl)ethyl)benzonitrile (2o). General procedure A was employed for the reaction of 4-bromobenzonitrile $(46 \mathrm{mg}, 0.25 \mathrm{mmol})$ with 2-(2oxo-3,4-dihydroquinolin-1(2H)-yl)propanoic acid $^{2}(82 \mathrm{mg}, \quad 0.375 \mathrm{mmol})$. The [Ni(dtbbpy)(H2O)4]Cl2 (5.8 mg, $0.0125 \mathrm{mmol})$ was used, which was added along with the $\operatorname{Ir}[\mathrm{dF}(\mathrm{CF} 3)$ ppy]2(dtbbpy)(PF6) $(5.0 \mathrm{mg}, 5.0 \mu \mathrm{mol})$. DMA $(2.5 \mathrm{~mL})$ and DBU $(57 \mu \mathrm{L}$, $0.375 \mathrm{mmol}$ ) were then added, and the reaction was stirred for 10 hours on the Penn photoreactor. This procedure afforded the title compound (31.5 mg, $46 \%)$, isolated via silica gel column chromatography (eluent hexanes and ethyl acetate) as a light yellow oil: ${ }^{1} \mathrm{H}$ NMR (600 MHz, DMSO-d6) $\delta 7.80$ (d, $J=8.4 \mathrm{~Hz}, 2 \mathrm{H}$ ), 7.47 (d, $\left.J=8.0 \mathrm{~Hz}, 2 \mathrm{H}\right), 7.24$ (d, $J=7.0 \mathrm{~Hz}, 1 \mathrm{H}), 7.04(\mathrm{t}, J=7.6 \mathrm{~Hz}, 1 \mathrm{H}), 6.95(\mathrm{t}, J=7.3 \mathrm{~Hz}, 1 \mathrm{H}), 6.63(\mathrm{~d}, J=8.1 \mathrm{~Hz}$, $1 \mathrm{H}), 6.13(\mathrm{q}, J=6.8 \mathrm{~Hz}, 1 \mathrm{H}), 2.95-2.84(\mathrm{~m}, 2 \mathrm{H}), 2.71-2.64(\mathrm{~m}, 1 \mathrm{H}), 2.63-2.56(\mathrm{~m}$, $1 \mathrm{H}), 1.74(\mathrm{~d}, J=7.1 \mathrm{~Hz}, 3 \mathrm{H}) .{ }^{13} \mathrm{C}$ NMR $(151 \mathrm{MHz}$, DMSO) $\delta 170.3,147.3,138.2,132.4$, 
$128.1,127.9,126.9,126.6,122.7,118.8,116.7,109.3,50.5,32.0,24.7,16.4$. HRMS (ESI+) $277.1354\left(277.1341\right.$ calcd for $\left.\mathrm{C}_{18} \mathrm{H}_{16} \mathrm{~N}_{2} \mathrm{O}, \mathrm{M}+\mathrm{H}^{+}\right)$.

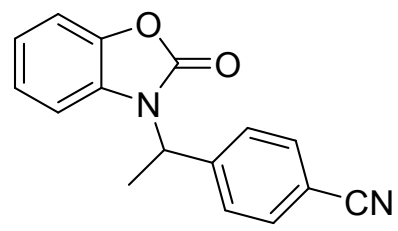

4-(1-(2-oxobenzo[d]oxazol-3(2H)-yl)ethyl)benzonitrile (2p). General procedure A was employed for the reaction of 4-bromobenzonitrile $(46 \mathrm{mg}, 0.25 \mathrm{mmol})$ with 2-(2oxobenzo[d]oxazol-3(2H)-yl)propanoic acid (78 $\mathrm{mg}, 0.375 \quad \mathrm{mmol})$. The [Ni(dtbbpy)(H2O)4]Cl2 (5.8 mg, $0.0125 \mathrm{mmol})$ was used, which was added along with the $\operatorname{Ir}[\mathrm{dF}(\mathrm{CF} 3)$ ppy]2(dtbbpy)(PF6) $(5.0 \mathrm{mg}, 5.0 \mu \mathrm{mol})$. DMA $(2.5 \mathrm{~mL})$ and DBU $(57 \mu \mathrm{L}$, $0.375 \mathrm{mmol}$ ) were then added, and the reaction was stirred for 10 hours on the Penn photoreactor. This procedure afforded the title compound (36.4 mg, 55\%), isolated via silica gel column chromatography (eluent hexanes and ethyl acetate) as a clear oil: ${ }^{1} \mathbf{H}$ NMR (600 MHz, DMSO-d6) $\delta 7.85$ (d, J = 8.4 Hz, 2H), 7.63 (d, J = 8.2 Hz, 2H), $7.38-$ $7.33(\mathrm{~m}, 1 \mathrm{H}), 7.14-7.07(\mathrm{~m}, 3 \mathrm{H}), 5.69(\mathrm{q}, J=7.2 \mathrm{~Hz}, 1 \mathrm{H}), 1.88(\mathrm{~d}, J=7.2 \mathrm{~Hz}, 3 \mathrm{H}) .{ }^{13} \mathrm{C}$ NMR (151 MHz, DMSO) $\delta 153.2,144.7,142.0,132.6,129.7,127.7,123.8,122.4,118.5,110.7$, 110.0, 109.9, 51.8, 16.7. HRMS (ESI+) 265.0975 (265.0977 calcd for $\mathrm{C}_{16} \mathrm{H}_{12} \mathrm{~N}_{2} \mathrm{O}_{2}, \mathrm{M}+\mathrm{H}^{+}$).

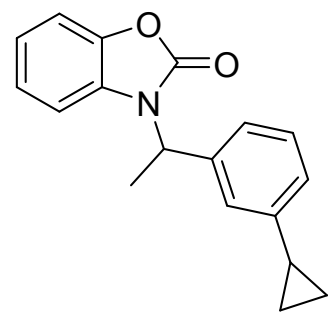

3-(1-(3-cyclopropylphenyl)ethyl)benzo[d]oxazol-2(3H)-one (2q). General procedure A was employed for the reaction of 1-bromo-3-cyclopropylbenzene $(49 \mathrm{mg}, 0.25 \mathrm{mmol}$ ) with 2-(2-oxobenzo[d]oxazol-3(2H)-yl)propanoic acid (78 mg, $0.375 \mathrm{mmol})$. The [Ni(dtbbpy)(H2O)4]Cl2 (11.7 mg, $0.025 \mathrm{mmol})$ was used, which was added along with the $\operatorname{Ir}[\mathrm{dF}(\mathrm{CF} 3)$ ppy]2(dtbbpy)(PF6) $(5.0 \mathrm{mg}, 5.0 \mu \mathrm{mol})$. DMA $(2.5 \mathrm{~mL})$ and DBU $(57 \mu \mathrm{L}$, 
$0.375 \mathrm{mmol}$ ) were then added, and the reaction was stirred for 10 hours on the Penn photoreactor. This procedure afforded the title compound (42.0 mg, 60\%), isolated via silica gel column chromatography (eluent hexanes and ethyl acetate) as an oil: ${ }^{1} \mathbf{H}$ NMR (600 MHz, DMSO-d6) ס 7.35 - $7.32(\mathrm{~m}, 1 \mathrm{H}), 7.25-7.21(\mathrm{~m}, 1 \mathrm{H}), 7.19-7.17(\mathrm{~m}, 2 \mathrm{H})$, $7.12-7.05(\mathrm{~m}, 3 \mathrm{H}), 6.96(\mathrm{~d}, \mathrm{~J}=7.6 \mathrm{~Hz}, 1 \mathrm{H}), 5.54(\mathrm{q}, \mathrm{J}=7.2 \mathrm{~Hz}, 1 \mathrm{H}), 1.92-1.87(\mathrm{~m}$, 1H), $1.85(\mathrm{~d}, \mathrm{~J}=7.2 \mathrm{~Hz}, 3 \mathrm{H}), 0.96-0.89(\mathrm{~m}, 2 \mathrm{H}), 0.67-0.59(\mathrm{~m}, 2 \mathrm{H}) .{ }^{13} \mathrm{C}$ NMR $(151$ MHz, DMSO) $\delta 153.3,144.2,141.9,139.1,129.8,128.6,124.3,124.1,123.7,123.5$, 122.2, 110.2, 109.8, 52.2, 17.0, 15.0, 9.6, 9.5. HRMS (ESI+) 280.1344 (280.1337 calcd for $\mathrm{C}_{18} \mathrm{H}_{17} \mathrm{NO}_{2}, \mathrm{M}+\mathrm{H}^{+}$).

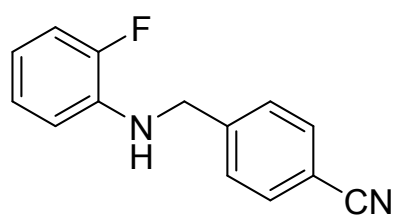

4-((2-fluorophenyl)amino)methyl)benzonitrile (2r). General procedure A was employed for the reaction of 4-bromobenzonitrile $(46 \mathrm{mg}, 0.25 \mathrm{mmol}$ ) with (2fluorophenyl)glycine (63 mg, $0.375 \mathrm{mmol})$. The [Ni(dtbbpy)(H2O)4]Cl2 (11.7 mg, 0.025 $\mathrm{mmol}$ ) was used, which was added along with the $\operatorname{Ir}[\mathrm{dF}$ (CF3)ppy]2(dtbbpy)(PF6) (2.5 mg, $2.5 \mu \mathrm{mol})$. DMA $(2.5 \mathrm{~mL})$ and DBU $(57 \mu \mathrm{L}, 0.375 \mathrm{mmol})$ were then added, and the reaction was stirred for 15 hours on the hepatochem PhotoRedOX Duo photoreactor which was equipped with two $40 \mathrm{~W}$ Kessil A160WE lamps at maximum intensity. This procedure afforded the title compound (50.4 mg, 89\%), isolated via silica gel column chromatography (eluent hexanes and ethyl acetate) as a light yellow oil: ${ }^{1} \mathbf{H}$ NMR (600 MHz, DMSO-d6) $\delta 7.77$ (d, J = 8.2 Hz, 2H), $7.54(\mathrm{~d}, J=8.2 \mathrm{~Hz}, 2 \mathrm{H}), 7.05-6.98(\mathrm{~m}, 1 \mathrm{H})$, $6.85(\mathrm{t}, J=7.7 \mathrm{~Hz}, 1 \mathrm{H}), 6.55-6.45(\mathrm{~m}, 2 \mathrm{H}), 6.40-6.32(\mathrm{~m}, 1 \mathrm{H}), 4.44(\mathrm{~d}, J=6.3 \mathrm{~Hz}$, 2H). ${ }^{13} \mathrm{C}$ NMR MHz, DMSO) $\delta 150.9$ (d, J=238.7 Hz), 146.3, 136.0 (d, J=11.9 Hz), 132.2, 127.8, 124,6, 118.9, 115.8 (d, J=6.8 Hz), 114.4 (d, J= $18.0 \mathrm{~Hz}$ ), 112.1 (d, J=3.7 Hz), 109.4, 45.5.HRMS (ESI+) $227.0986\left(227.0984\right.$ calcd for $\left.\mathrm{C}_{14} \mathrm{H}_{11} \mathrm{FN}_{2}, \mathrm{M}+\mathrm{H}^{+}\right)$. 
<smiles>N#Cc1ccc(CN2CC(=O)Oc3ccccc32)cc1</smiles>

\section{4-((2-oxo-2,3-dihydro-4H-benzo[b][1,4]oxazin-4-yl)methyl)benzonitrile}

General procedure A was employed for the reaction of 4-bromobenzonitrile (46 mg, 0.25 $\mathrm{mmol}$ ) with 2-(2-oxo-2,3-dihydro-4H-benzo[b][1,4]oxazin-4-yl)acetic acid (77 mg, 0.375 $\mathrm{mmol})$. The $[\mathrm{Ni}(\mathrm{dtbbpy})(\mathrm{H} 2 \mathrm{O}) 4] \mathrm{Cl} 2(11.7 \mathrm{mg}, 0.025 \mathrm{mmol})$ was used, which was added along with the $\operatorname{Ir}[\mathrm{dF}(\mathrm{CF} 3)$ ppy]2(dtbbpy)(PF6) $(2.5 \mathrm{mg}, 2.5 \mu \mathrm{mol})$. DMA (2.5 mL) and DBU $(57 \mu \mathrm{L}, 0.375 \mathrm{mmol}$ ) were then added, and the reaction was stirred for 15 hours on the hepatochem PhotoRedOX Duo photoreactor which was equipped with two $40 \mathrm{~W}$ Kessil A160WE lamps at maximum intensity. This procedure afforded the title compound (48.4 $\mathrm{mg}, 73 \%$ ), isolated via silica gel column chromatography (eluent hexanes and ethyl acetate) as an oil: ${ }^{1} \mathrm{H}$ NMR (600 MHz, DMSO-d6) $\delta 7.81(\mathrm{~d}, \mathrm{~J}=8.0 \mathrm{~Hz}, 2 \mathrm{H}), 7.55(\mathrm{~d}, \mathrm{~J}=$ $7.9 \mathrm{~Hz}, 2 \mathrm{H}), 7.07$ (d, J = 7.7 Hz, 1H), $6.99(\mathrm{t}, \mathrm{J}=7.6 \mathrm{~Hz}, 1 \mathrm{H}), 6.82(\mathrm{t}, \mathrm{J}=7.5 \mathrm{~Hz}, 1 \mathrm{H})$, $6.75(\mathrm{~d}, \mathrm{~J}=7.9 \mathrm{~Hz}, 1 \mathrm{H}), 4.56(\mathrm{~s}, 2 \mathrm{H}), 4.10(\mathrm{~s}, 2 \mathrm{H}) .{ }^{13} \mathrm{C}$ NMR MHz, DMSO) $\delta$ 164.7, 143.0, $141.1,134.3,132.5,128.3,125.0,119.3,118.7,116.4,113.2,110.0,52.1,50.1$. HRMS (ESI+) 265.0966 (265.0977 calcd for $\mathrm{C}_{16} \mathrm{H}_{12} \mathrm{~N}_{2} \mathrm{O}_{2}, \mathrm{M}+\mathrm{H}^{+}$).

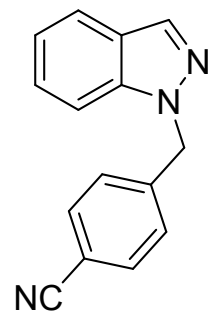

4-((1H-indazol-1-yl)methyl)benzonitrile (2t). General procedure A was employed for the reaction of 4-bromobenzonitrile $(46 \mathrm{mg}, 0.25 \mathrm{mmol})$ with $2-(1 \mathrm{H}$-indazol-1-yl)acetic acid (66 mg, $0.375 \mathrm{mmol})$. The [Ni(dtbbpy)(H2O)4]Cl2 (5.8 mg, $0.0125 \mathrm{mmol})$ was used, which was added along with the $\operatorname{Ir}[\mathrm{dF}(\mathrm{CF} 3)$ ppy]2(dtbbpy)(PF6) $(5.0 \mathrm{mg}, 5.0 \mu \mathrm{mol})$. DMA $(2.5 \mathrm{~mL})$ and $\mathrm{DBU}(57 \mu \mathrm{L}, 0.375 \mathrm{mmol})$ were then added, and the reaction was stirred for 12 hours on the Penn photoreactor. This procedure afforded the title compound (30.8 $\mathrm{mg}, 53 \%$ ), isolated via silica gel column chromatography (eluent hexanes and ethyl acetate) as a light yellow oil: ${ }^{1} \mathrm{H}$ NMR $(600 \mathrm{MHz}, \mathrm{DMSO}-\mathrm{d} 6) \delta 8.53(\mathrm{~s}, 1 \mathrm{H}), 7.81$ (d, $J=$ 
$8.3 \mathrm{~Hz}, 2 \mathrm{H}), 7.72(\mathrm{~d}, J=8.4 \mathrm{~Hz}, 1 \mathrm{H}), 7.60(\mathrm{~d}, J=8.7 \mathrm{~Hz}, 1 \mathrm{H}), 7.43(\mathrm{~d}, J=8.4 \mathrm{~Hz}, 2 \mathrm{H})$, $7.26-7.20(\mathrm{~m}, 1 \mathrm{H}), 7.07-7.01(\mathrm{~m}, 1 \mathrm{H}), 5.77(\mathrm{~s}, 2 \mathrm{H}) .{ }^{13} \mathrm{C} \mathrm{NMR} \mathrm{MHz}$, DMSO) $\delta 148.5$, $142.5,132.5,128.5,125.7,124.6,121.5,121.2,120.7,118.6,117.0,110.6,55.6 . H R M S$ (ESI+) $234.1039\left(234.1031\right.$ calcd for $\left.\mathrm{C}_{15} \mathrm{H}_{11} \mathrm{~N}_{3}, \mathrm{M}+\mathrm{H}^{+}\right)$.

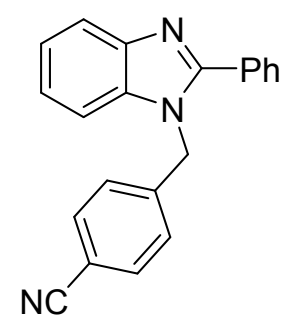

4-((2-phenyl-1H-benzo[d]imidazol-1-yl)methyl)benzonitrile (2u). General procedure A was employed for the reaction of 4-bromobenzonitrile $(46 \mathrm{mg}, 0.25 \mathrm{mmol})$ with 2-(2phenyl-1H-benzo[d]imidazol-1-yl)acetic acid (94 $\mathrm{mg}, \quad 0.375 \quad \mathrm{mmol})$. The [Ni(dtbbpy) $(\mathrm{H} 2 \mathrm{O}) 4] \mathrm{Cl} 2 \quad(5.8 \mathrm{mg}, 0.0125 \mathrm{mmol})$ was used, which was added along with the $\operatorname{Ir}[\mathrm{dF}(\mathrm{CF} 3)$ ppy]2(dtbbpy)(PF6) $(2.5 \mathrm{mg}, 2.5 \mu \mathrm{mol})$. DMA $(2.5 \mathrm{~mL})$ and DBU $(57 \mu \mathrm{L}$, $0.375 \mathrm{mmol}$ ) were then added, and the reaction was stirred for 15 hours on the hepatochem PhotoRedOX Duo photoreactor which was equipped with two $40 \mathrm{~W}$ Kessil A160WE lamps at maximum intensity. This procedure afforded the title compound (35.0 $\mathrm{mg}, 45 \%$ ), isolated via silica gel column chromatography (eluent hexanes and ethyl acetate) as a white solid: ${ }^{1} \mathrm{H}$ NMR $(600 \mathrm{MHz}$, DMSO-d6) $\delta 7.79-7.73(\mathrm{~m}, 3 \mathrm{H}), 7.72-$ $7.66(\mathrm{~m}, 2 \mathrm{H}), 7.54-7.48(\mathrm{~m}, 3 \mathrm{H}), 7.46(\mathrm{~d}, J=7.4 \mathrm{~Hz}, 1 \mathrm{H}), 7.30-7.21(\mathrm{~m}, 2 \mathrm{H}), 7.17(\mathrm{~d}$, $J=8.3 \mathrm{~Hz}, 2 \mathrm{H}), 5.69(\mathrm{~s}, 2 \mathrm{H}) .{ }^{13} \mathrm{C}$ NMR MHz, DMSO) $\delta 153.2,142.7,142.6,135.8,132.7$, 129.9, 129.9, $129.0128 .8,127.0,122.9,122.4,119.4,118.5,110.9,110.3,47.2$. HRMS (ESI+) 310.1356 (310.1344 calcd for $\mathrm{C}_{21} \mathrm{H}_{15} \mathrm{~N}_{3}, \mathrm{M}+\mathrm{H}^{+}$).

\section{Synthesis of $\boldsymbol{N}$-linked acid substrates:}

Although we acquired $\mathrm{N}$-linked acids from commercial suppliers or our internal building block inventory for this paper, we wanted to show how easy it is to make them from cheap starting materials. Here we show the synthesis and yields for two different substrates. HNMR (in dmso-d6) and purity LCMS traces are included at the end of the SI for reference as well. 


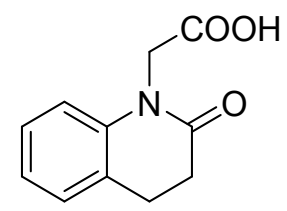

2-(2-oxo-3,4-dihydroquinolin-1(2H)-yl)acetic acid (1a): Sodium hydride $(60 \%$ in mineral oil, $11.0 \mathrm{mmol}, 440 \mathrm{mgs}, 1.1$ equiv.) was added to a dried round bottom flask and placed under nitrogen. To this was added dry DMF (30 mL). The 3,4-dihydroquinolin$2(1 \mathrm{H})$-one (10 mmol, $1470 \mathrm{mgs}, 1.0$ equiv.) was added portion wise to this stirring mixture. It was allowed to stir at room temperature for 1 hour and then ethyl 2-bromoacetate (12.5 mmol, $1.392 \mathrm{~mL}, 1.25$ equiv.) was added dropwise over 5 minutes. When done as noted by LCMS, the reaction mixture was cooled to $0{ }^{\circ} \mathrm{C}$ and then quenched with $1 \mathrm{~mL}$ of wet isopropanol followed by $5 \mathrm{~mL}$ of water and it was stirred for 30 minutes at room temperature. After this time, it was evaporated in vacuo and used as is for the next step.

The above compound was dissolved in THF and water $(1: 1,40 \mathrm{~mL})$. Then lithium hydroxide ( $15.0 \mathrm{mmol}, 360 \mathrm{mgs}, 1.50$ equiv.) was added to it and it was allowed to stir for 5 hours or until done as noted by LCMS. It was then concentrated in vacuo to remove the THF and water was added to achieve a volume around $35 \mathrm{~mL}$. This was then extracted $1 \times 15 \mathrm{~mL}$ of DCM (while the mixture was still basic), and the organic layer was discarded. Then the aqueous layer was made acidic $(\sim p \mathrm{pH} 3)$ by the addition of $6 \mathrm{M} \mathrm{HCl}$ and it was extracted 3x $100 \mathrm{~mL}$ of Ethyl Acetate. The organic layers were combined, dried with magnesium sulfate, and concentrated in vacuo to afford the title compound as a light yellow solid ( $77 \%$ yield, $1.58 \mathrm{~g})$. This material was pure as is and used for the flow reaction. ${ }^{1} \mathrm{H}$ NMR (600 MHz, DMSO-d6) $\delta 12.90$ (br s, 1H), $7.25-7.20(\mathrm{~m}, 2 \mathrm{H}), 7.00$ (t, $\mathrm{J}=7.4 \mathrm{~Hz}, 1 \mathrm{H}), 6.91(\mathrm{~d}, \mathrm{~J}=7.9 \mathrm{~Hz}, 1 \mathrm{H}), 4.56(\mathrm{~s}, 2 \mathrm{H}), 2.92-2.84(\mathrm{~m}, 2 \mathrm{H}), 2.60-2.54$ $(\mathrm{m}, 2 \mathrm{H})$.

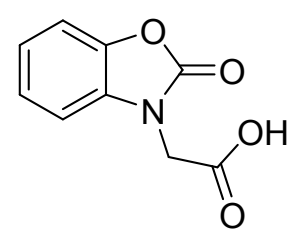

2-(2-oxobenzo[d]oxazol-3(2H)-yl)acetic acid: This title compound was synthesized as noted in the above procedure except that benzo[d]oxazol-2(3H)-one $(9.32 \mathrm{mmol}, 1260$ 
mgs, 1.0 equiv.) was used as the amine precursor. After the workup as noted above, this material contained around a $7 \%$ impurity, so it was subjected to silica gel column chromatography (Hexanes and Ethyl Acetate) to further purify the material. After chromatography the title compound was afforded as a brown solid in $61 \%$ yield $(1.10 \mathrm{~g})$. ${ }^{1} \mathrm{H}$ NMR (600 MHz, DMSO-d6) ठ $13.17(\mathrm{~s}, 1 \mathrm{H}), 7.38(\mathrm{~d}, \mathrm{~J}=7.9 \mathrm{~Hz}, 1 \mathrm{H}), 7.31$ (d, J = 7.8 $\mathrm{Hz}, 1 \mathrm{H}), 7.23(\mathrm{t}, \mathrm{J}=7.7 \mathrm{~Hz}, 1 \mathrm{H}), 7.16(\mathrm{t}, \mathrm{J}=7.8 \mathrm{~Hz}, 1 \mathrm{H}), 4.65(\mathrm{~s}, 2 \mathrm{H})$.

\section{Flow setup:}

All flow reactions were performed in a Vapourtec UV-150 photoreactor attached to a Vapourtec R-Series flow system equipped with an autosampler. The entire continuous flow system is under inert atmosphere during the course of the reaction. An image and schematic of the set-up used is provided below in Figure S1. Dimethylacetamide (DMA) used as the flow solvent was purchased in $1 \mathrm{~L}$ Sure-Seal ${ }^{\mathrm{TM}}$ bottles from Sigma Aldrich and degassed prior to use by bubbling nitrogen for $15 \mathrm{~min}$.

A

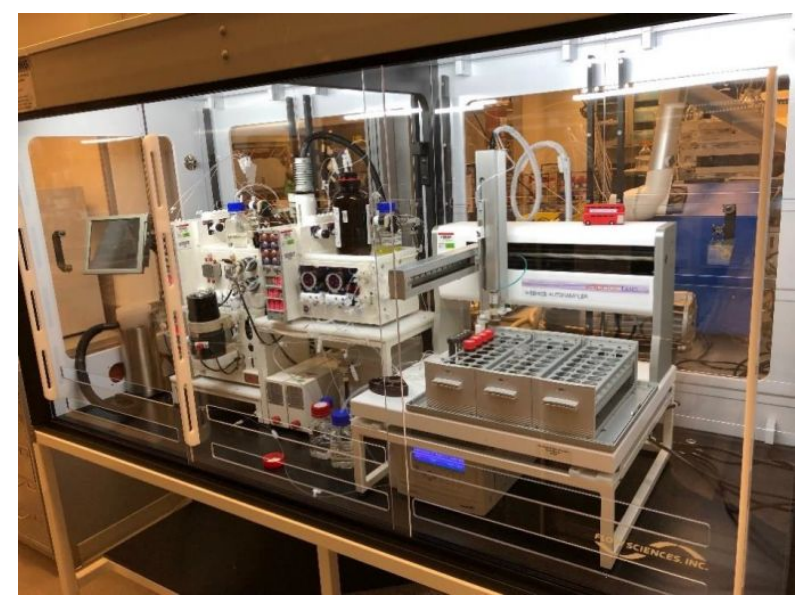




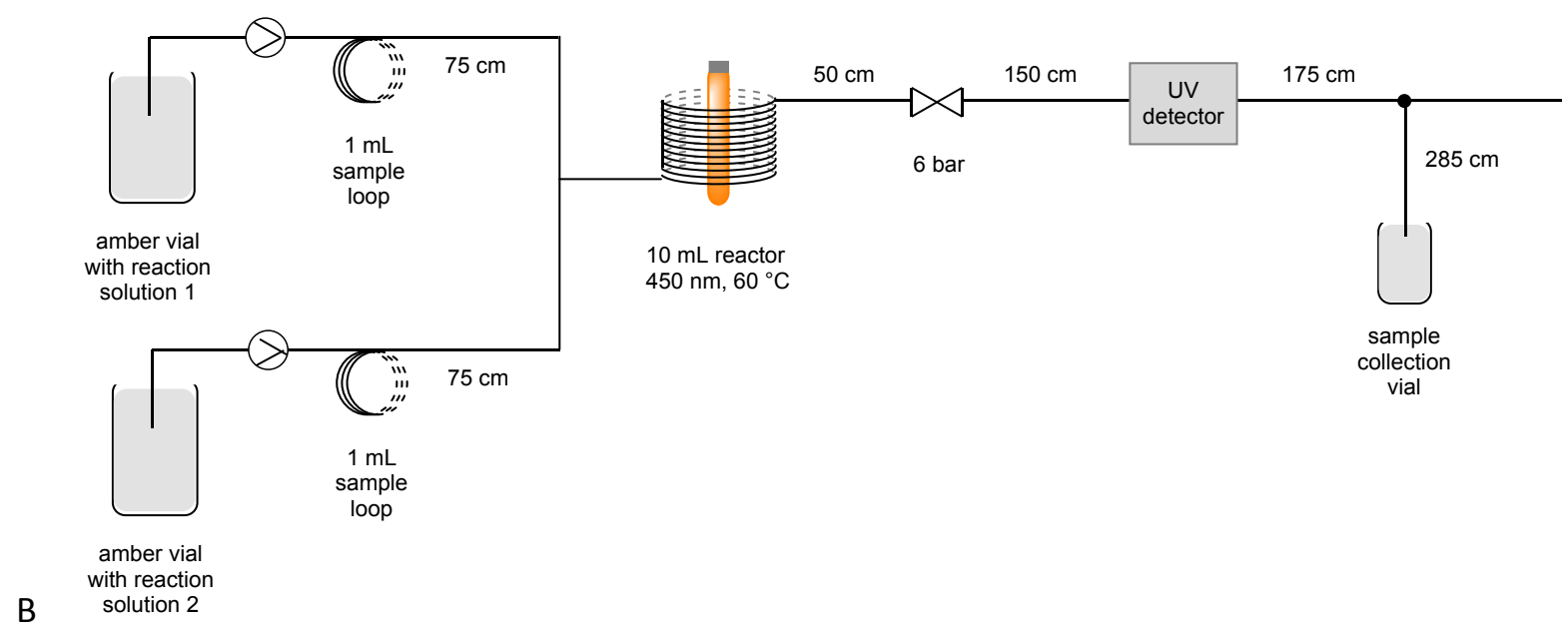

Figure S1 - (A) Photograph and (B) Schematic of the Vapourtec R-Series setup.

General Procedure for the decarboxylative cross coupling under continuous flow conditions for synthesis of $\mathbf{2 k}$ : Two stock solutions were prepared; (1) 2-(2,3-dimethyl7-oxothieno[2,3- $d$ ]pyridazin-6(7H)-yl)acetic acid (1250 mg, $5.25 \quad \mathrm{mmol}), \quad 4-$

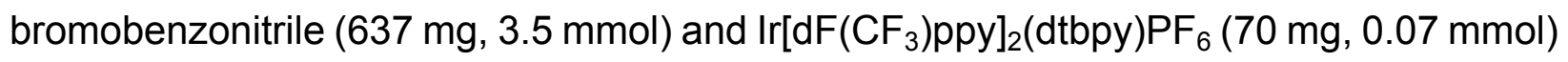
in anhydrous DMA $(30 \mathrm{~mL})$ and $(2)\left[\mathrm{Ni}(\mathrm{dtbbpy})(\mathrm{H} 2 \mathrm{O})_{4}\right] \mathrm{Cl}_{2}(82 \mathrm{mg}, 0.175 \mathrm{mmol})$ and $1,8-$ diazabicyclo[5.4.0]undec-7-ene (888 $\mu \mathrm{l}, 5.25 \mathrm{mmol})$ in anhydrous DMA (30 mL). The reaction mixtures were degassed by bubbling argon through the resulting solution for 5 minutes. If solid particles remained after this process, the reaction mixtures were briefly sonicated to assist the solvation. An argon balloon was left on the reaction vials and the materials were placed on the instrument.

The Vapourtec R-Series flow reactor equipped with a UV- $15010 \mathrm{~mL}$ photoreactor was pre-equilibrated with anhydrous DMA. A 10 bar back pressure regulator was used to maintain a stable system pressure. The R2S pump $C$ and pump $D$ (peristaltic) were used to continuously inject the stock solutions 1 and 2 via $1 \mathrm{~mL}$ sample loops and pumped through combining prior to passing through the $10 \mathrm{~mL}$ Vapourtec UV-150 Photoreactor ( $1.3 \mathrm{~mm}$ inner diameter, 0.15 wall thickness, fluoropolymer tube) at $60^{\circ} \mathrm{C}$ and 20 minutes residence time, irradiating with a blue LED array (450 nm, lamp power 100\%, 24 watts). The reaction plug was followed through the reactor by anhydrous DMA and collection was triggered by UV detection and the dispersion model created by the Flow Commander application. The reaction mixture was collected in a $100 \mathrm{~mL}$ flask on the output rack of the flow reactor. The output mixture was analyzed by LCMS, then concentrated to remove 
DMA. It was purified on silica gel and then with SFC (Biphenyl 21x250 mm) to afford the desired material (2k) as a white solid (235 mgs).

Flow conditions for the synthesis of $2 \mathrm{a}$ : Flow conditions to access product $2 \mathrm{a}$ were the same as note above, however only one solution was made and it was pumped from one single reaction mixture which contained the following. 2-(2-oxo-3,4-dihydroquinolin1(2H)-yl)acetic acid (1380 mg, $6.77 \mathrm{mmol})$, 4-bromobenzonitrile (910 mg, $5 \mathrm{mmol}$ ), $\operatorname{Ir}\left[\mathrm{dF}\left(\mathrm{CF}_{3}\right) \text { ppy }\right]_{2}(\mathrm{dtbpy}) \mathrm{PF}_{6}(50 \mathrm{mg}, 0.05 \mathrm{mmol}), \quad\left[\mathrm{Ni}(\mathrm{dtbbpy})(\mathrm{H} 2 \mathrm{O})_{4}\right] \mathrm{Cl}_{2}(230 \mathrm{mg}, 0.5$ $\mathrm{mmol}$ ) and 1,8-diazabicyclo[5.4.0]undec-7-ene (1140 $\mu \mathrm{l}, 6.77 \mathrm{mmol})$ in anhydrous DMA $(66.6 \mathrm{~mL})$. When done, the collected mixture was evaporated in vacuo and purified via silica gel column chromatography with hexanes and ethyl acetate to afford the desired product as a white solid (915 mgs, $70 \%$ ).

\section{Optimization trials for $\mathbf{2 k}$ and general comments on optimization of these reactions:}

In Scheme 3, example $2 \mathrm{k}$ had the lowest yield for the paper at $20 \%$. The reaction halts at $\sim 20 \%$ yield regardless of the conditions we tried to improve upon this. Whereas most of the $\mathrm{N}$-linked acids in this paper worked with a kessil lamp, in the hepatochem photoreactor, and in the PennOC/Merck photoreactor, use of the PennOC/Merck photoreactor was needed to access product $2 \mathrm{k}$, as the other blue light systems were not powerful enough in terms of light intensity. With how much acid remained at the end of these reactions (about $1 / 2$ of the 1.5 equiv.), the need for the PennOC/Merck photoreactor for this product is likely due to the need for a more powerful blue light source as this acid is particularly slow at converting under these conditions.

Also, the different acids in this paper have varying oxidation potentials, so it is possible that a different photocatalyst could be more optimal to access product $2 \mathrm{k}$. However, after screening 22 different photocatalyst only 5 of them afforded desired product $2 \mathrm{k}$ by LCMS, and none of them were better than the conditions noted in scheme 3 (see following table, the ratio of the product to internal standard was calculated compared to an internal biphenyl standard which all reactions received the same amount). 


\section{Photocatalyst Screen for 2k}

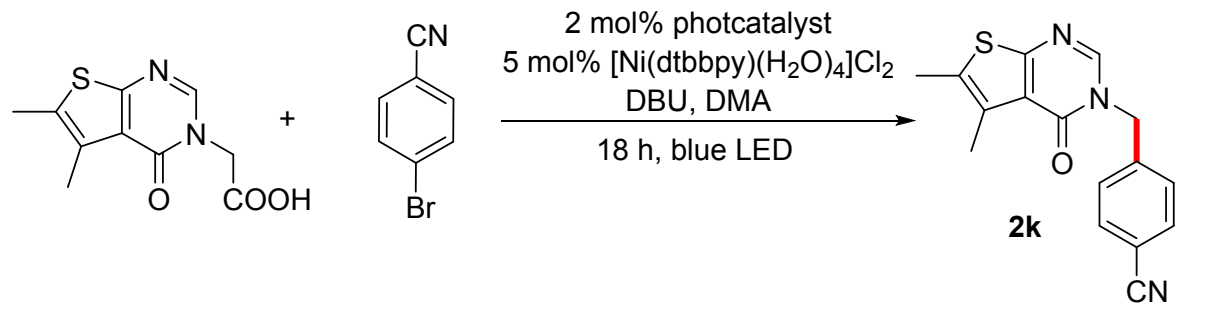

Photocatalyst

1.) $\operatorname{Ir}[\mathrm{dF}(\mathrm{CF} 3) \mathrm{ppy}] 2$ (dtbbpy)PF6

Ratio 2k:internal standard (From LCMS)

2.) $4-\mathrm{CZIPN}$

0.84

3.) $\operatorname{Ir}[\mathrm{dF}(\mathrm{F}) \mathrm{ppy}] 2$ (dtbpy)PF6

4.) $\operatorname{Ir}[\mathrm{dF}(\mathrm{H}) \mathrm{ppy}] 2$ (dtbpy)PF6

0.38

5.) $\operatorname{Ir}[\mathrm{dF}(\mathrm{Me}) \mathrm{ppy}] 2(\mathrm{dtbpy}) \mathrm{PF} 6$

6.) No catalyst

7.) No catalyst, no light

Also, the ratio of the iridium and nickel metal to each other can be very important. If the two cycles get out of sync, i.e. one is happening faster than the other this can lead to poor yields due to homocoupling of the radicals, debromination, etc..... It also could be useful to change to limiting reagent. For example, in the optimal conditions, we were using 1.5 equiv. of the acid to get product $2 \mathrm{k}$ and 1.0 equiv. of the 4-bromobenzonitrile. However, since acid was remaining, we switched to using 1.0 equiv. of the acid and 2 equiv. of the 4-bromobenzonitrile to try and drive the reaction to completion. Under these conditions even with doubling the catalyst and adding the second portion of the catalyst after 2 hours or increasing the Ir and $\mathrm{Ni}$ catalyst loading to $10 \%$ and $25 \%$ respectively, no improvement in yield was noted and most of the acid remained as noted in the LCMS trace.

Further, altering the conditions in flow to access product $2 \mathrm{k}$ did little to improve the yield either, see the following table for examples testing different conditions in flow on a $0.25 \mathrm{mmol}$ scale. Also, the crude LCMS after the optimal flow conditions (entry 1 below) is provided for context of how the reaction proceeds. Note the acid remaining and product $2 \mathrm{k}$ are reported as the AUC's from each of the crude LCMS traces after standardizing with an internal biphenyl standard. As noted below, no other ratios of Ir:Ni improved upon the $2 \%$ Ir and $5 \% \mathrm{Ni}$ from entry one. Other bases and temperatures were tested as well and were not superior to $\mathrm{DBU}$ and $60^{\circ} \mathrm{C}$. 


\section{Flow Optimization}

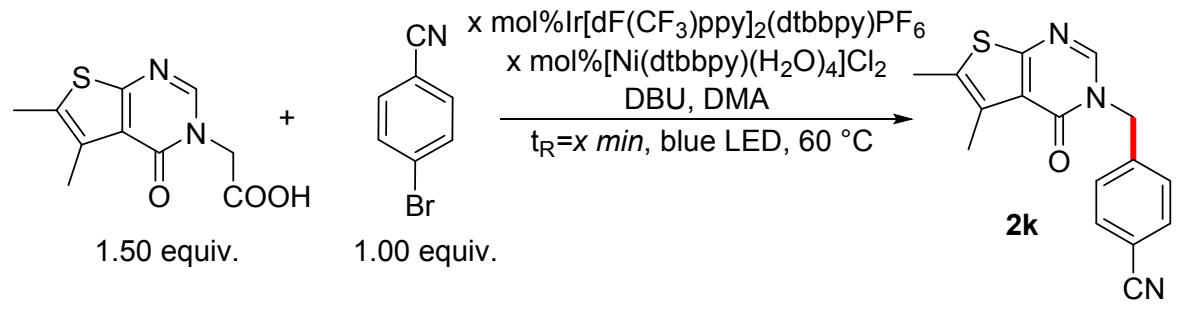

Conditions in flow

1.) $2 \% \mathrm{Ir}, 5 \% \mathrm{Ni}, \mathrm{t}_{\mathrm{R}}=60 \mathrm{~min}$

2.) $2 \% \mathrm{Ir}, 5 \% \mathrm{Ni}, \mathrm{t}_{\mathrm{R}}=30 \mathrm{~min}$

3.) $4 \% \operatorname{Ir}, 5 \% \mathrm{Ni}, t_{R}=60 \mathrm{~min}$

4.) $1 \% \mathrm{Ir}, 10 \% \mathrm{Ni}, \mathrm{t}_{\mathrm{R}}=60 \mathrm{~min}$

5.) $1 \% \mathrm{Ir}, 1 \% \mathrm{Ni}, \mathrm{t}_{\mathrm{R}}=30 \mathrm{~min}$

6.) $2 \% \mathrm{Ir}, 1 \% \mathrm{Ni}, \mathrm{t}_{\mathrm{R}}=30 \mathrm{~min}$
Standardized Product and Standardized Acid

. $230(2 \mathrm{k}) ; 210(\mathrm{COOH})$

$161(2 \mathrm{k}) ; 372(\mathrm{COOH})$

$214(2 \mathrm{k}) ; 217(\mathrm{COOH})$

$136(2 \mathrm{k}) ; 290(\mathrm{COOH})$

$63(2 \mathrm{k}) ; 375(\mathrm{COOH})$

$85(2 \mathrm{k}) ; 315(\mathrm{COOH})$

Standardized refers to the AUC in the LCMS after it was standardized for the added internal standard

\section{Crude LCMS after flow conditions from entry 1 above:}

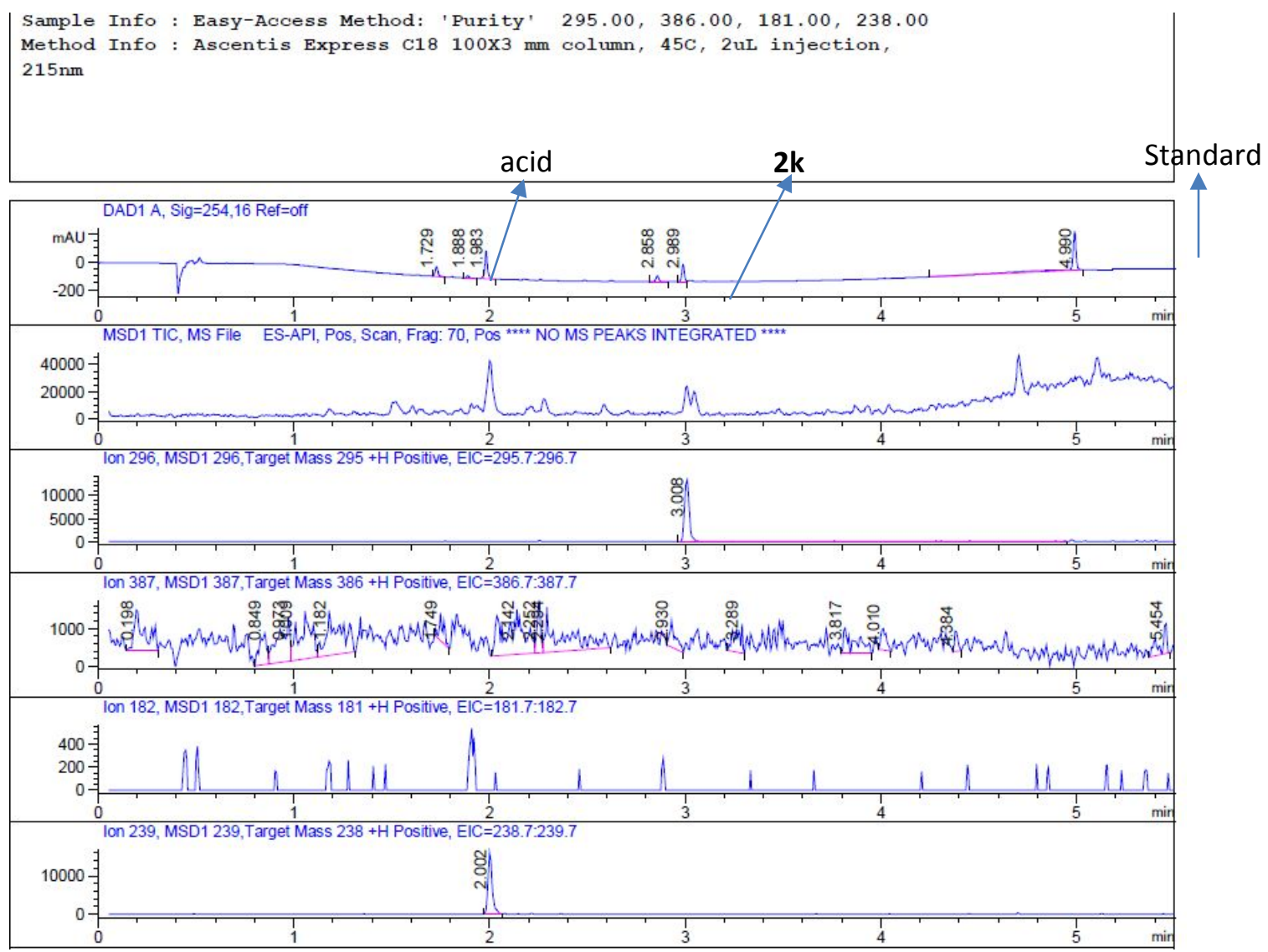


Other aryl halide SAR and discussion:
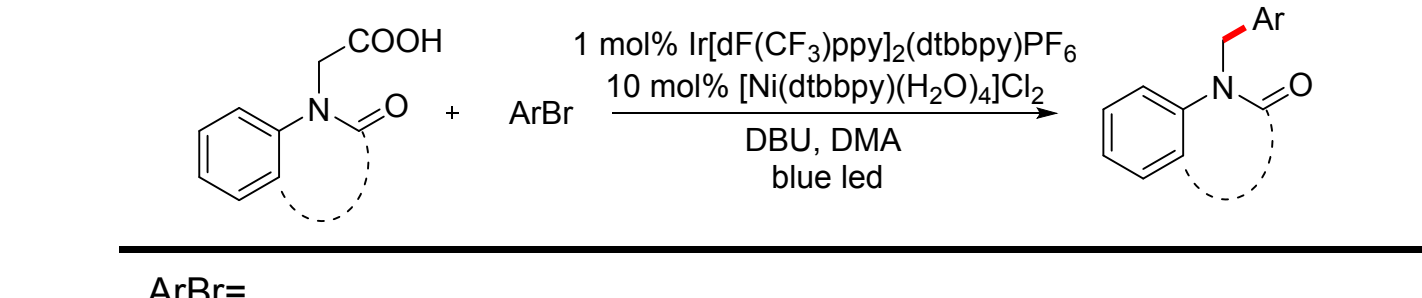<smiles>NS(=O)(=O)c1ccc(Br)cc1F</smiles>

$80 \%$<smiles>NC(=O)C1(c2cccc(Br)c2)CC(F)(F)C1</smiles><smiles>Clc1ccc(Br)cn1</smiles>

$67 \%$<smiles>OC(c1ccc(Br)cc1)C(F)(F)F</smiles>

$63 \%$<smiles>N#Cc1cc(Cl)cc(Br)c1</smiles>

$60 \%$<smiles>Cc1cn[nH]c1-c1cccc(Br)c1</smiles>

$43 \%$<smiles>COc1ccc(Br)cc1S(N)(=O)=O</smiles>

$33 \%$<smiles>FC(F)(F)c1ccc2cc(Br)ncc2c1</smiles><smiles>Cc1cccc2nc(C(F)(F)F)c(Br)n12</smiles>

$10 \%$<smiles>Brc1cccc(-c2nnc(C3CC3)o2)c1</smiles><smiles>FC(F)c1cc(Br)cc(C(F)F)n1</smiles><smiles>O=[N+]([O-])c1[nH]ccc1C(F)(F)F</smiles><smiles>CC(C)(C)[Na]</smiles>

$\mathrm{Br}$<smiles>Cc1ccn2c(C(F)(F)F)ncc2c1</smiles>

$7 \%$<smiles>CCOC(=O)c1cccc(Br)c1</smiles>

$0 \%$<smiles>Brc1ccc(C2CCN2)cc1</smiles>

Along with the SAR of aryl halides we included in the manuscript, we wanted to share some understanding of the SAR from this reaction that we discovered running some internal libraries $(\mathrm{COOH}$ is proprietary but similar to ones used in manuscript). The yields 
above refer to reaction ran on a $0.035 \mathrm{mmol}$ scale isolated by mass directed reverse phase purification. In general, the reaction tolerates fluorine, alkyl groups, ethers, esters, cyano, amides (free and substituted, sulfonamides (free and substituted), pyridines and other heteroaromatics. The reaction also is tolerant of free alcohols and amines off the aromatic rings, although we have had more success with alcohols opposed to amines. The reaction is also very tolerant to 5 membered heteroaromatic rings coming off the 6 membered aryl halides. In terms of aryl halides that are difficult in general we found the following to be true. Ortho substitutions can led to low yields (especially when going beyond just a plain fluorine). 5-membered heteroaromatic bromides typically do not work well in this reaction. Monosubstituted 4-membered ring lactams and azetidines are usually not tolerated in this reaction. Beyond these specifics, a lot of varied SAR works, and this reaction has proven to be extremely robust in our hands.

\section{References:}

1.) Gutiérrez-Bonet, A.; Tellis, J. C.; Matsui, J. K.; Vara, B. A.; Molander, G. A. ACS Catal., 2016, 6, 8004-8008.

2.) Fang, Y.-Q; Bio, M. M.; Hansen, K. B.; Potter, M. S.; Clausen, A. J. Am. Chem. Soc. 2010, 132, 15525-15527. 

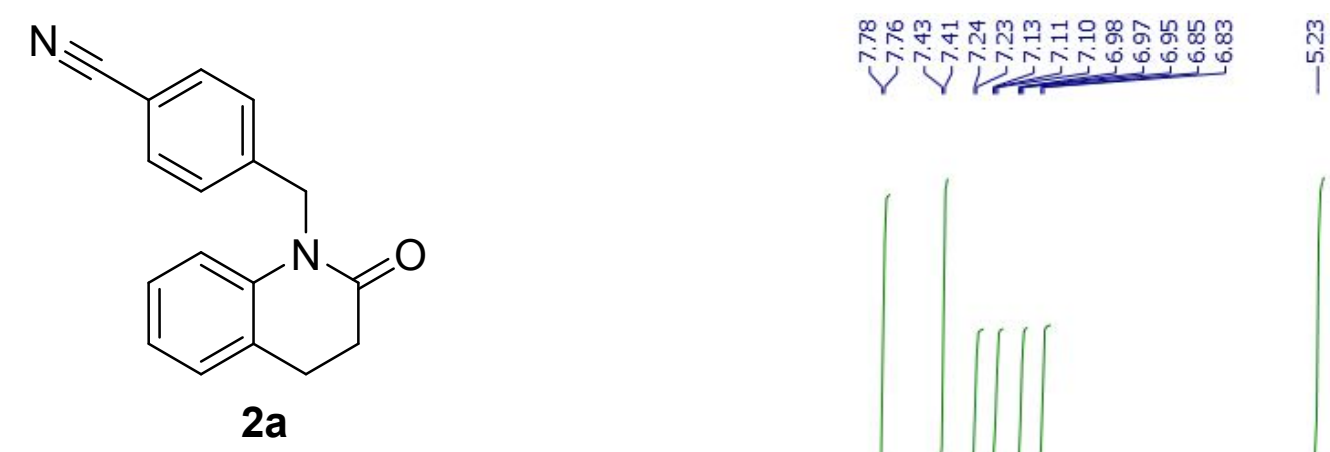

पूक्ष

MNNNNNN
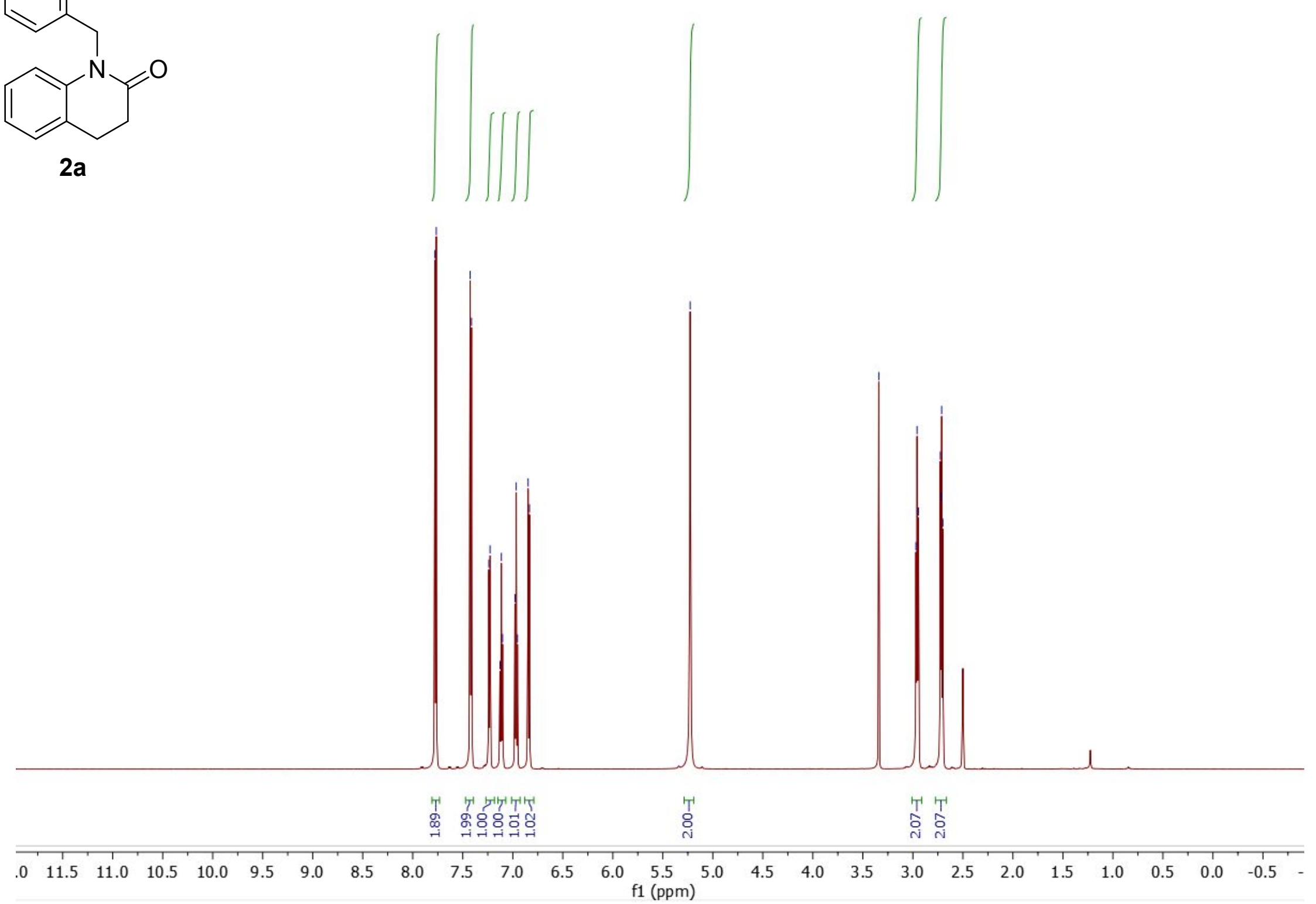

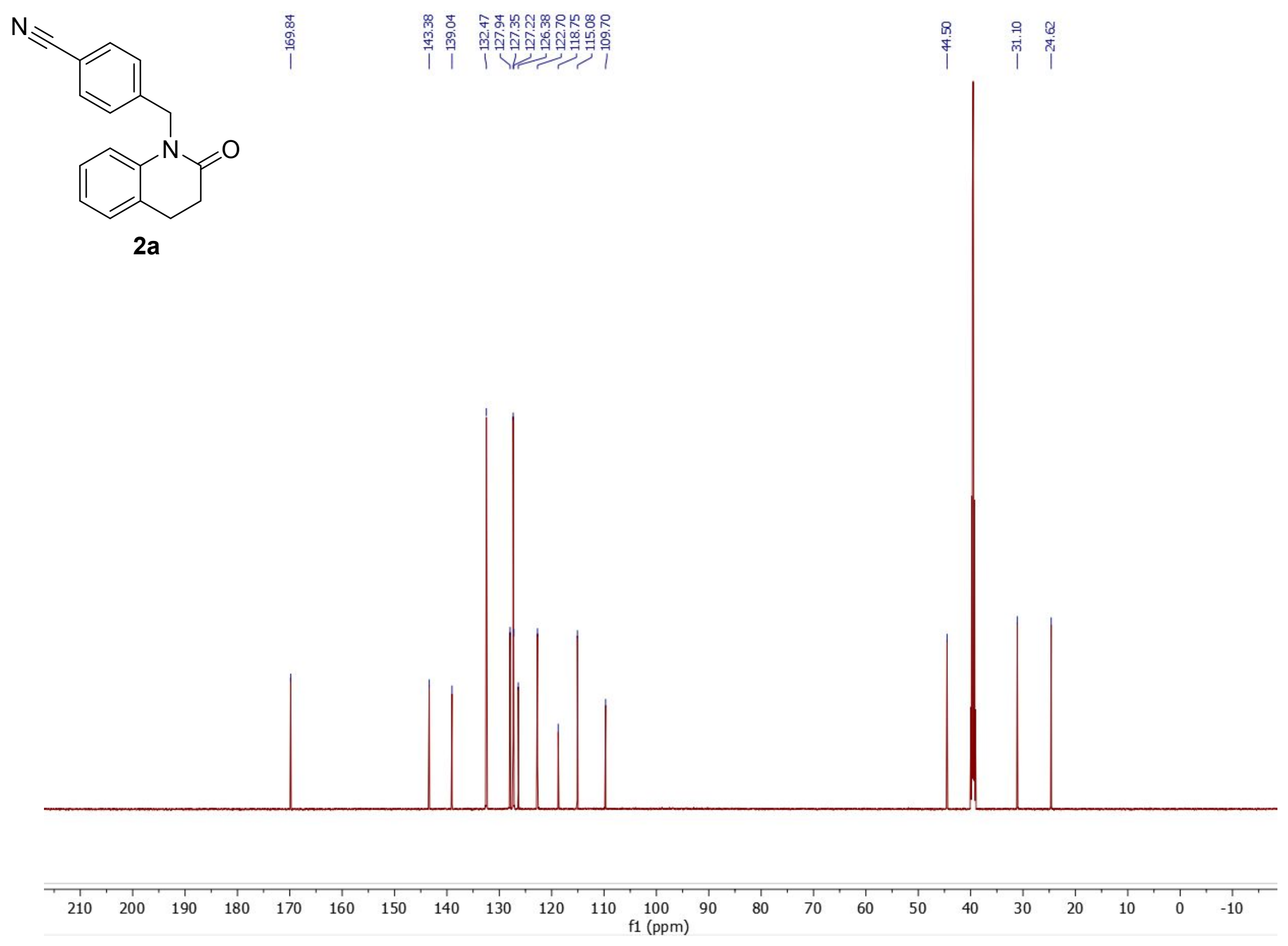


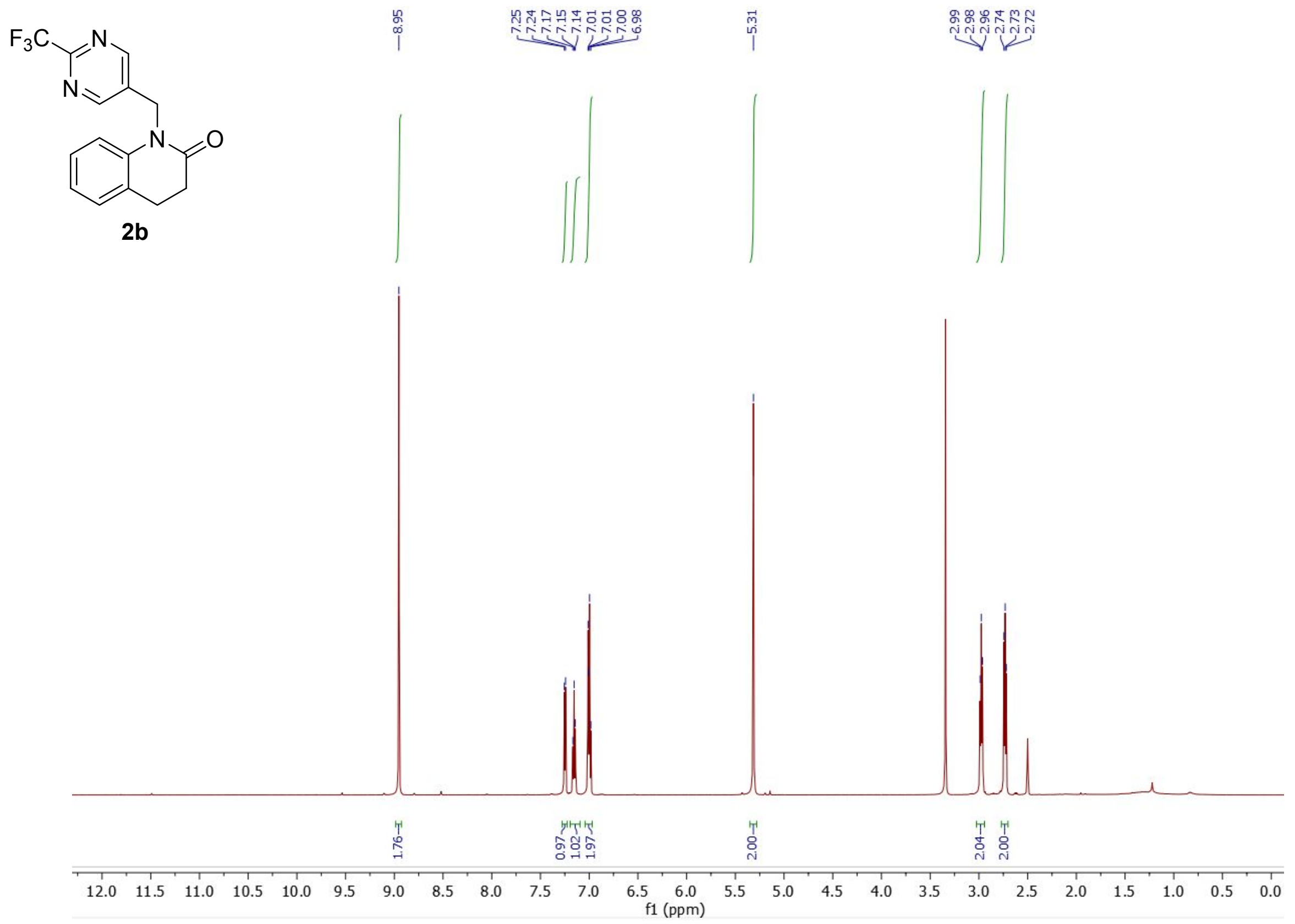




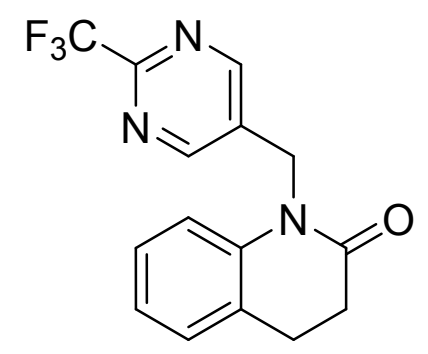

2b

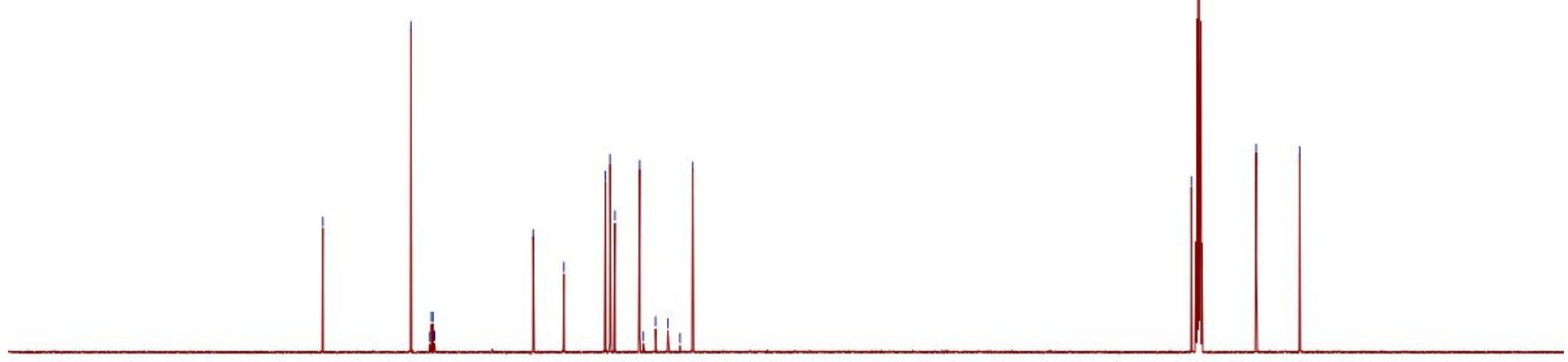

$\begin{array}{llllllllllllllllllllllll}210 & 200 & 190 & 180 & 170 & 160 & 150 & 140 & 130 & 120 & 110 & 100 & 90 & 80 & 70 & 60 & 50 & 40 & 30 & 20 & 10 & 0 & -10\end{array}$ 

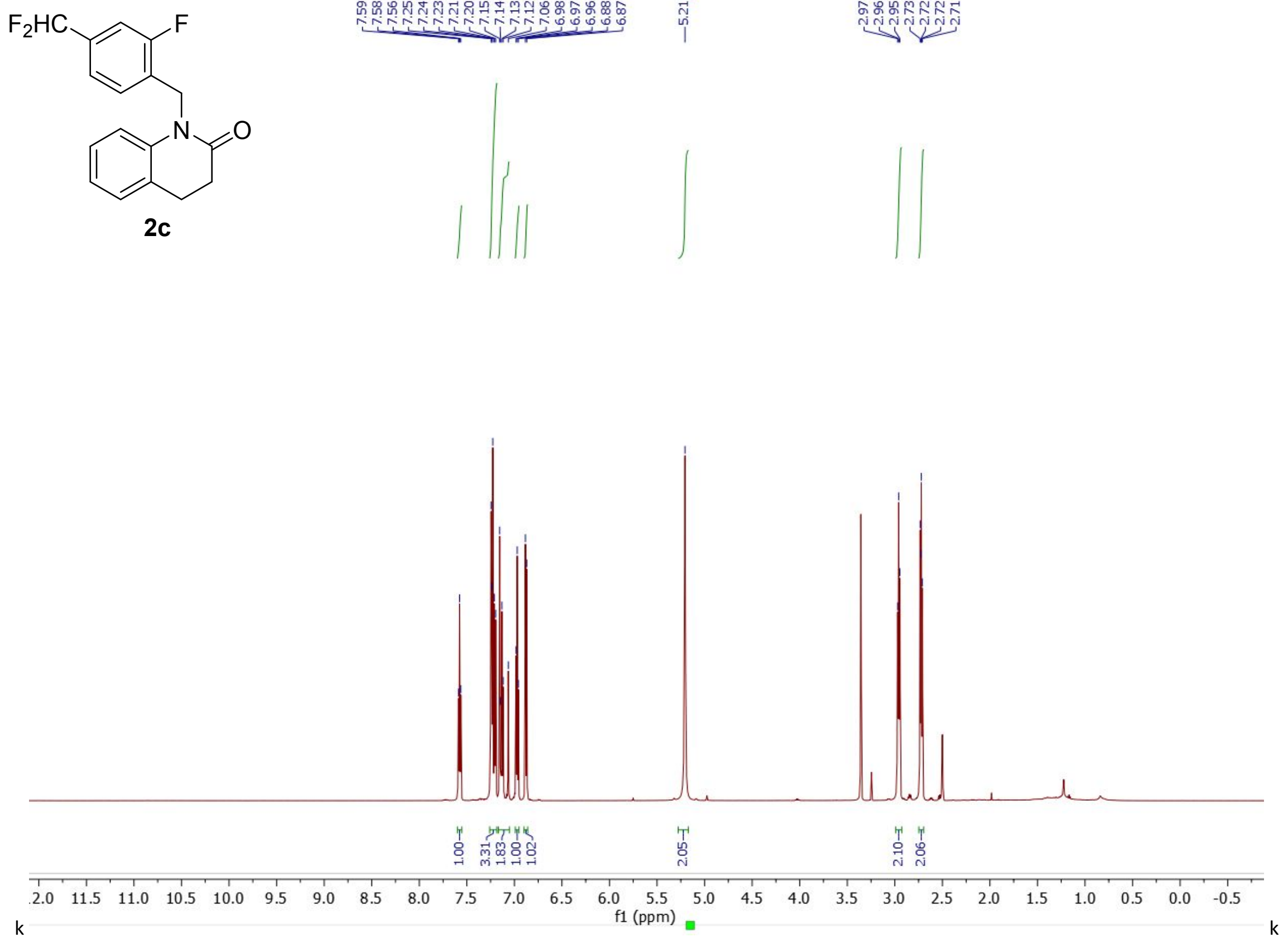

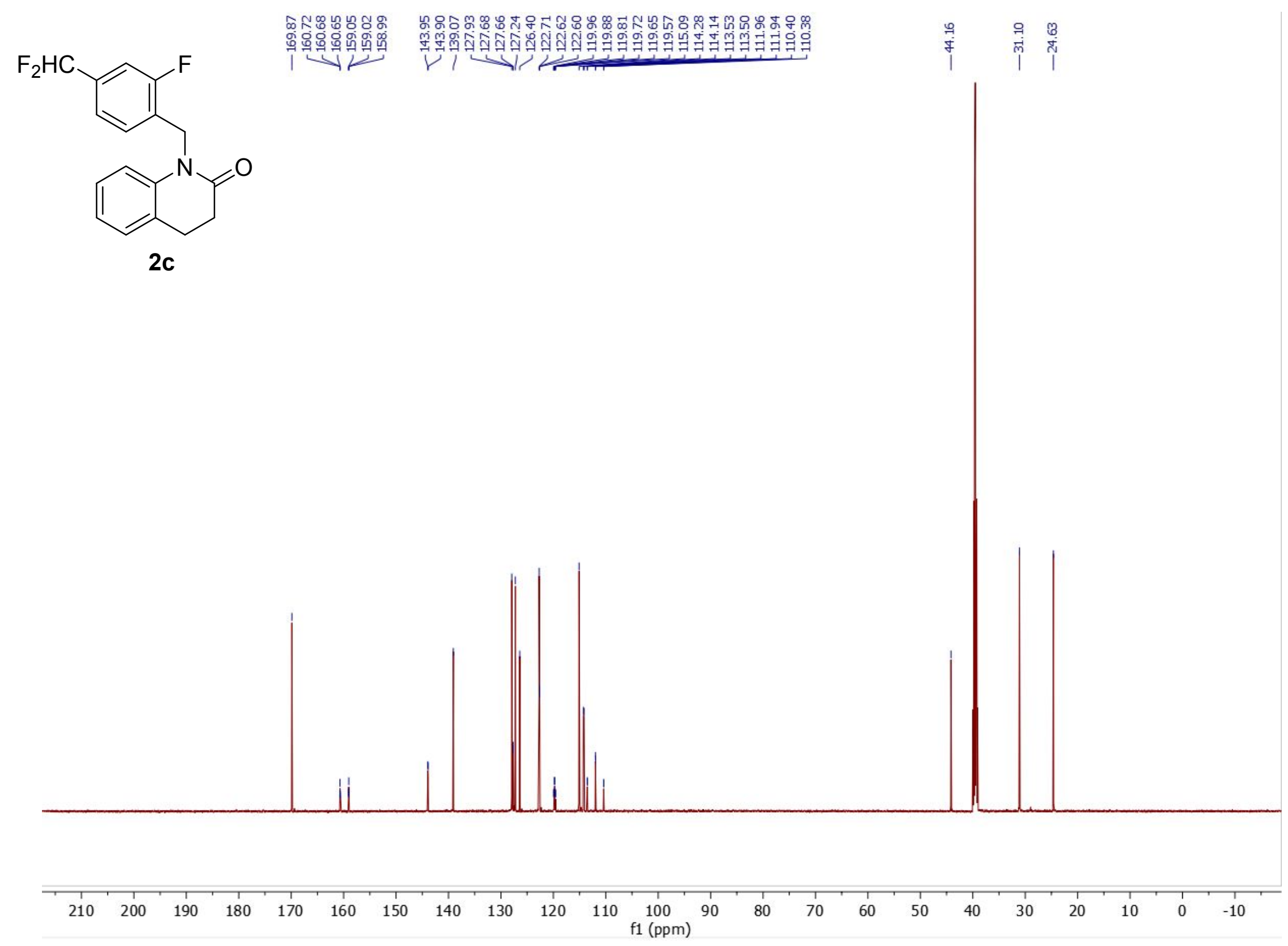


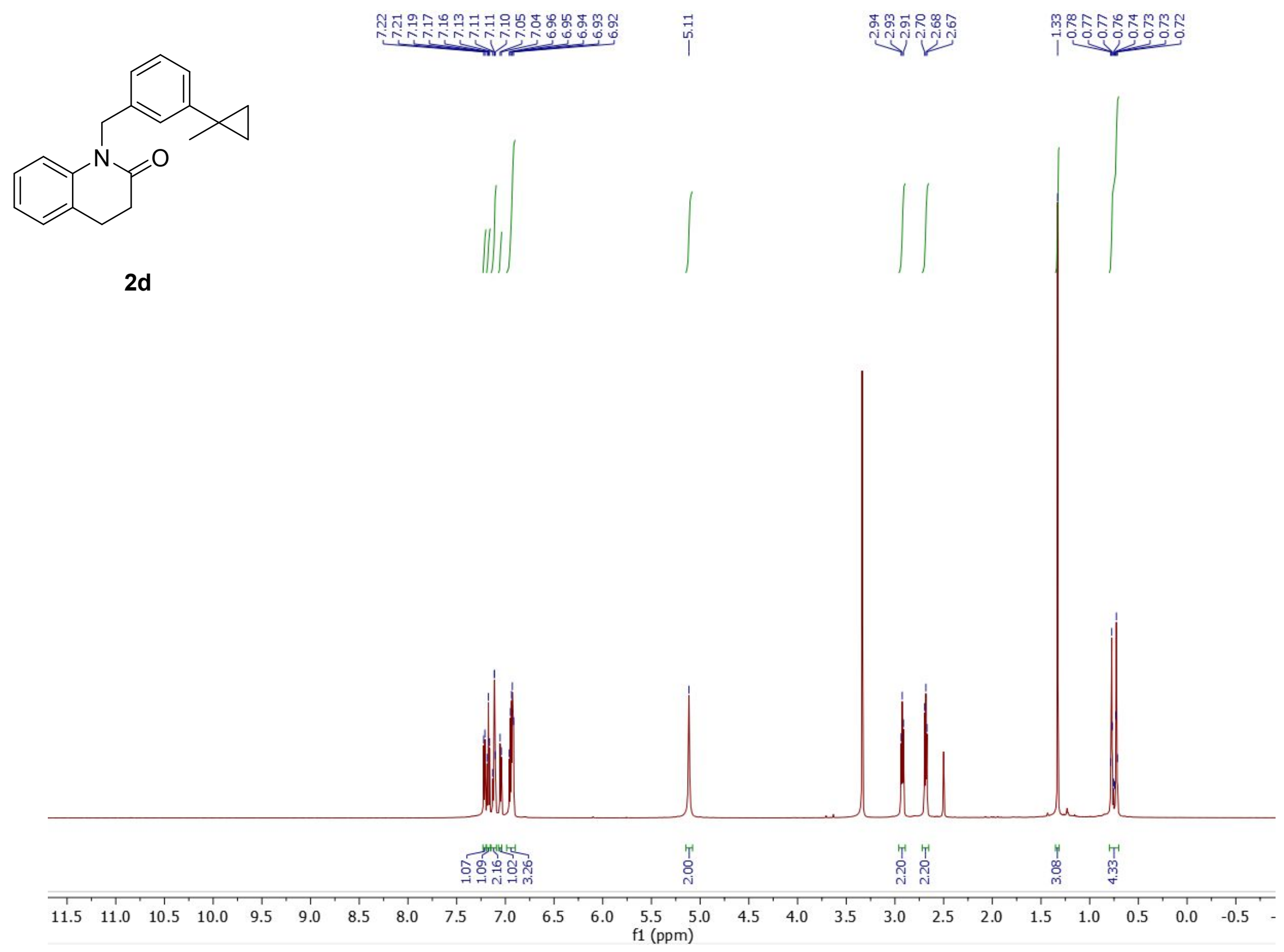




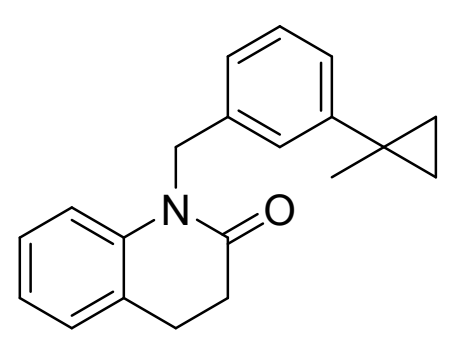

$2 d$

(1)

d
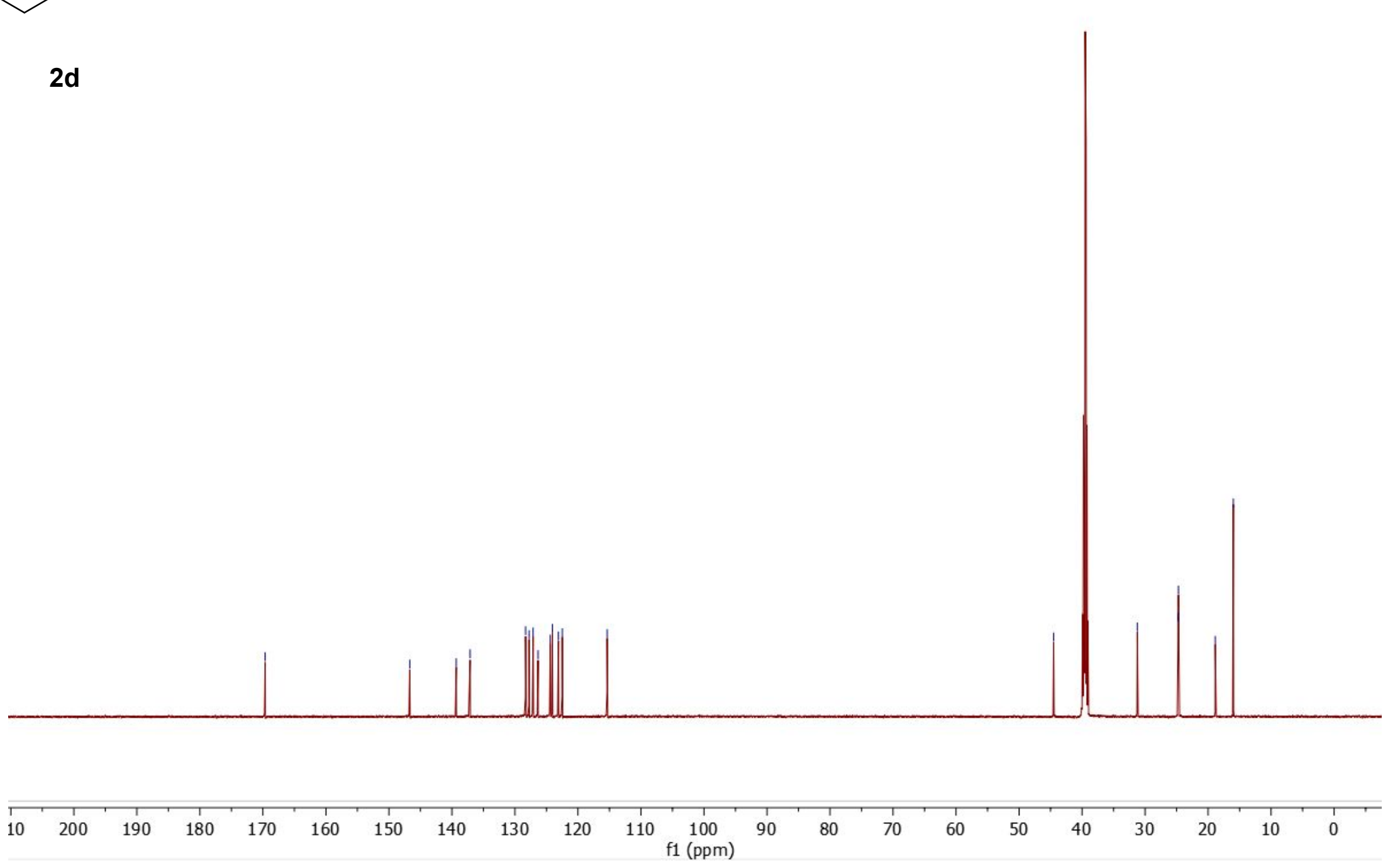


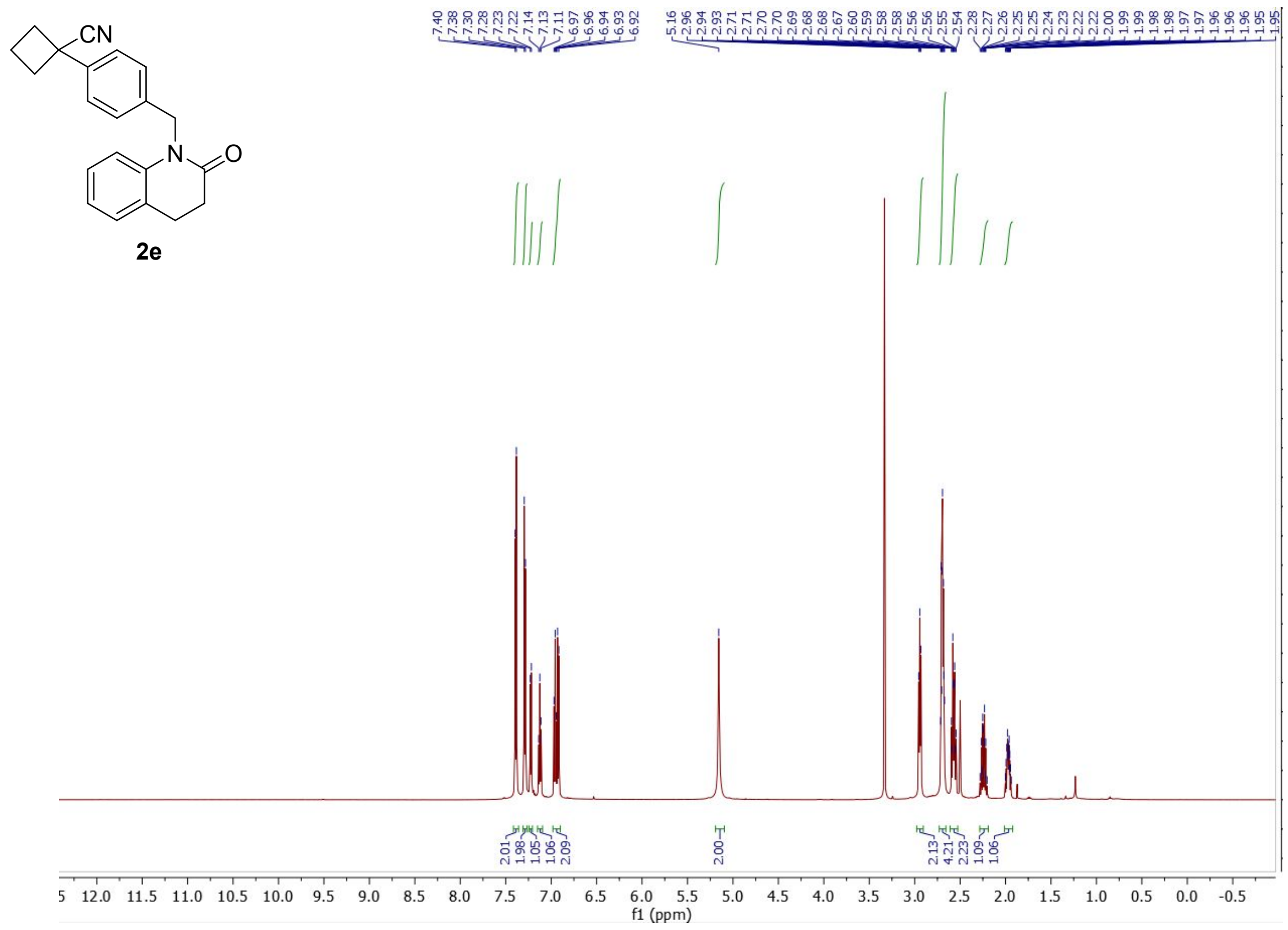




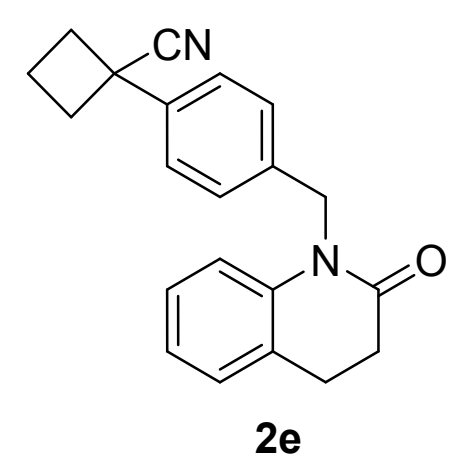

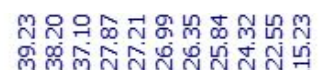

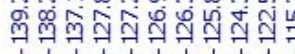

I.

$2 e$

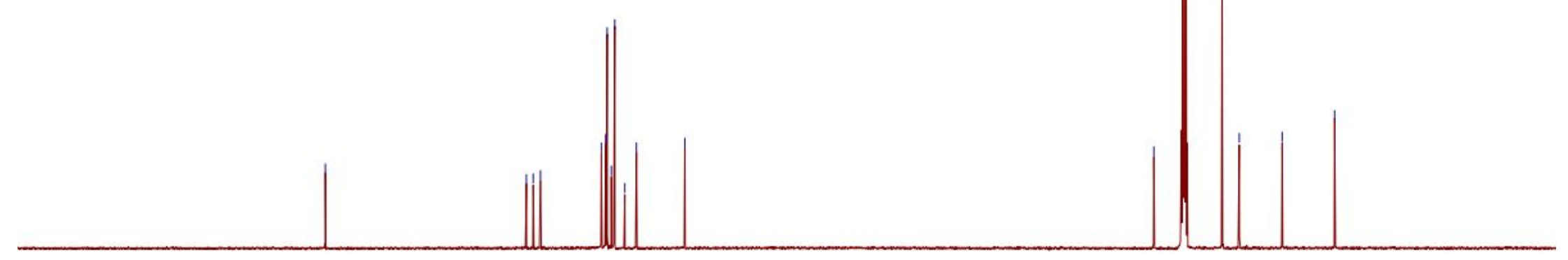

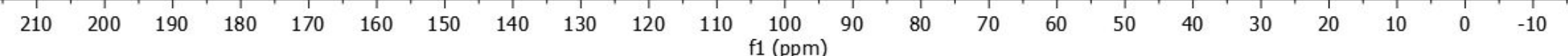




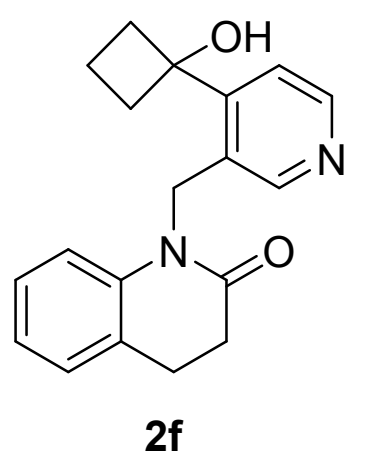

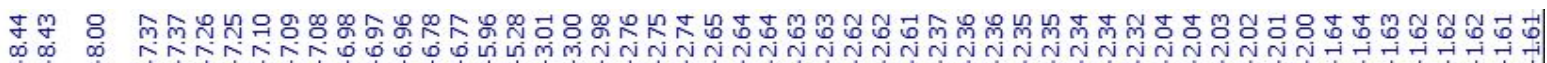

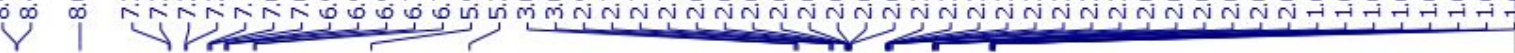
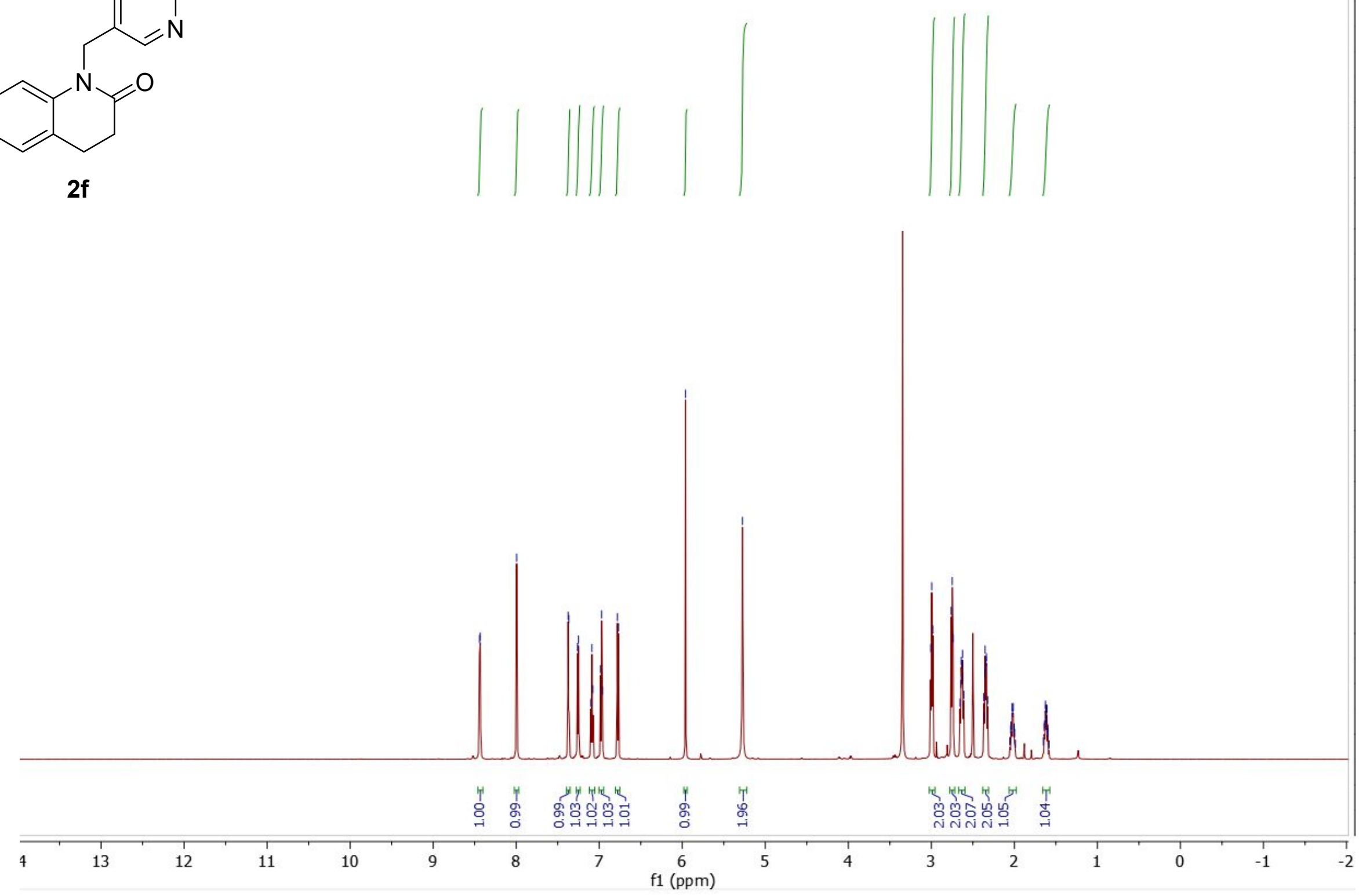

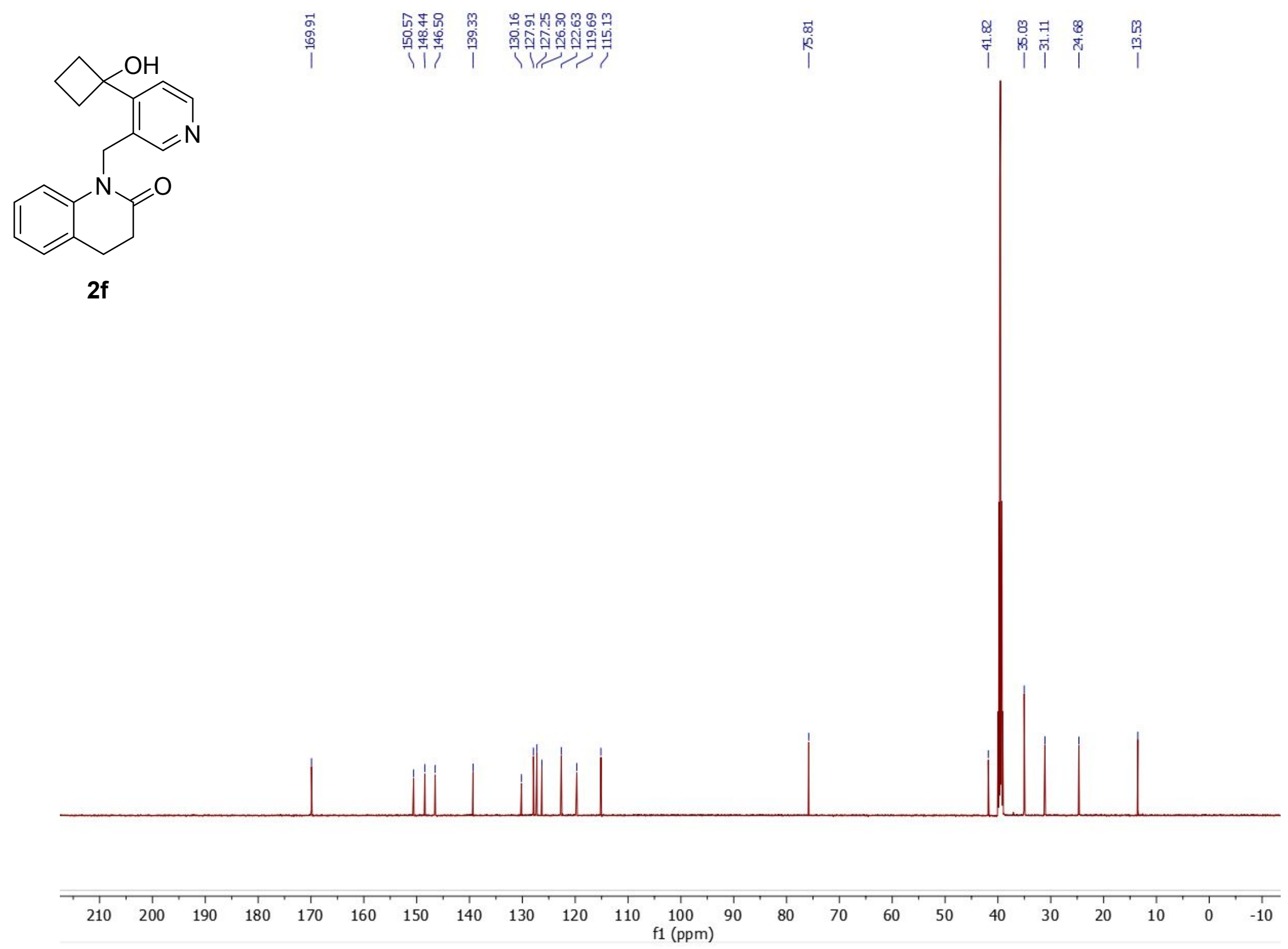


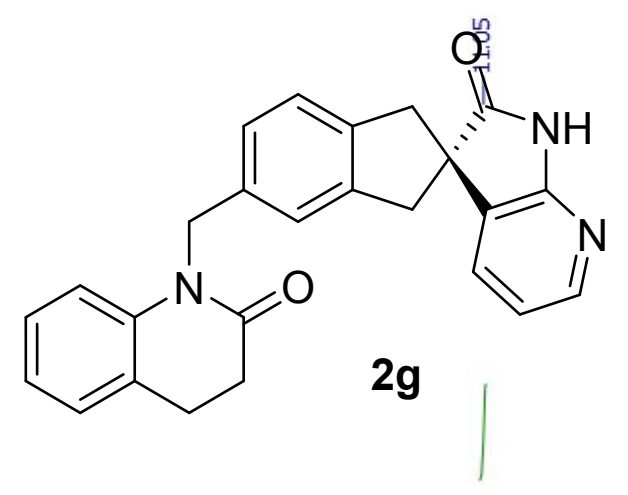

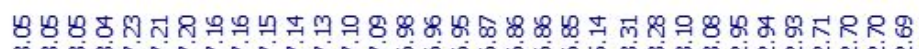

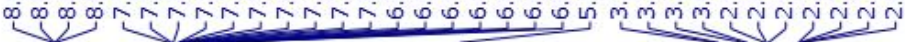

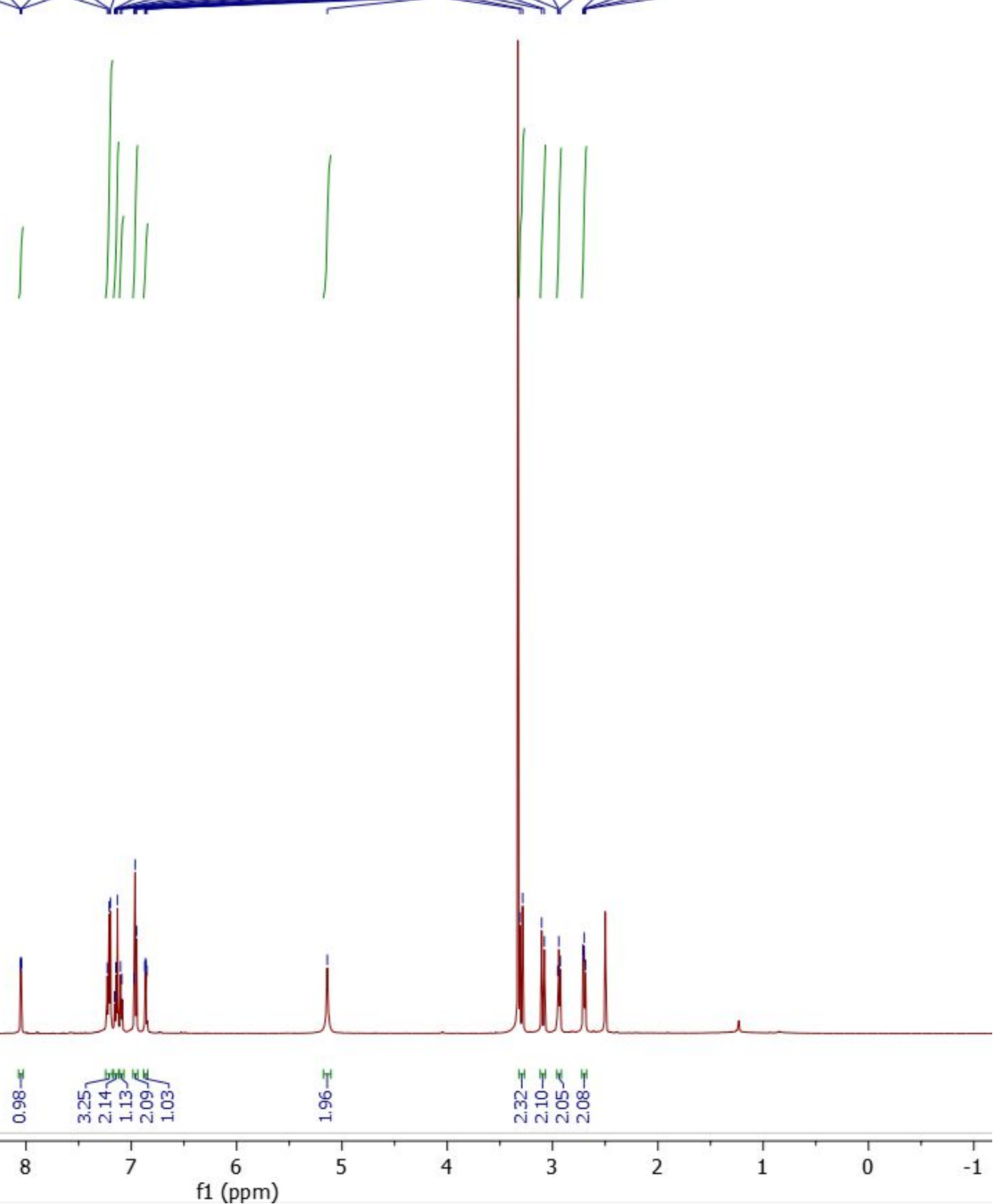

k

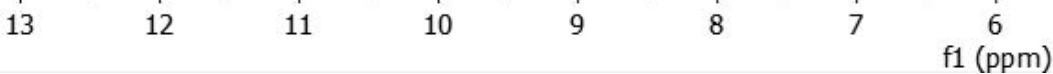




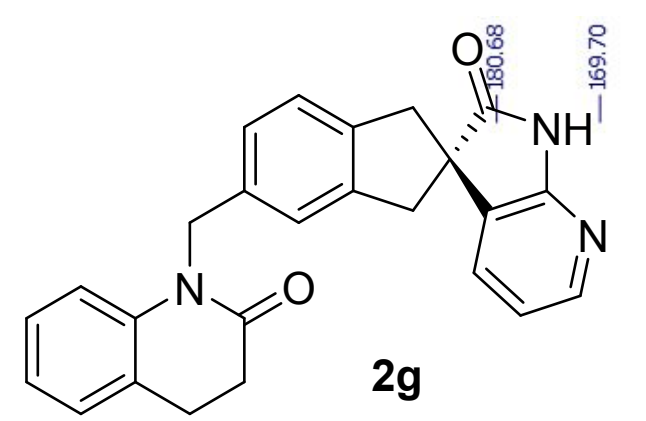

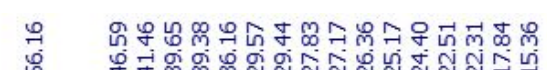

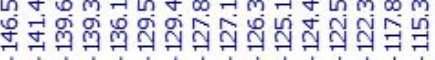

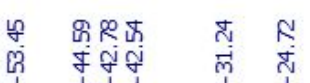

I Vर, पा

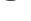
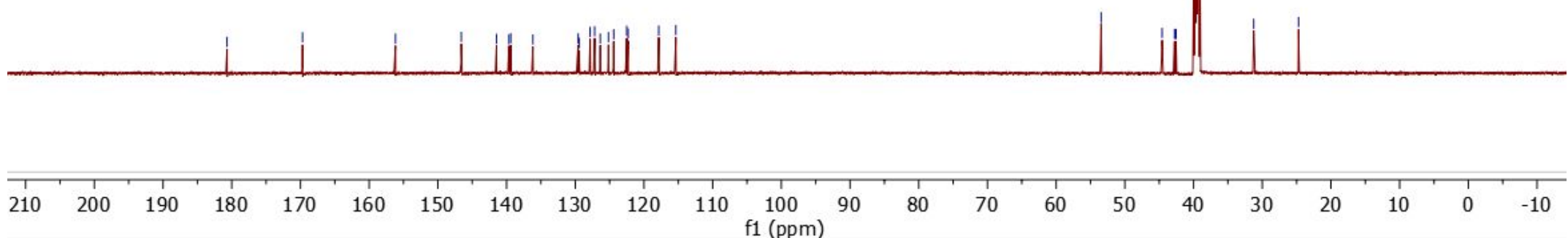


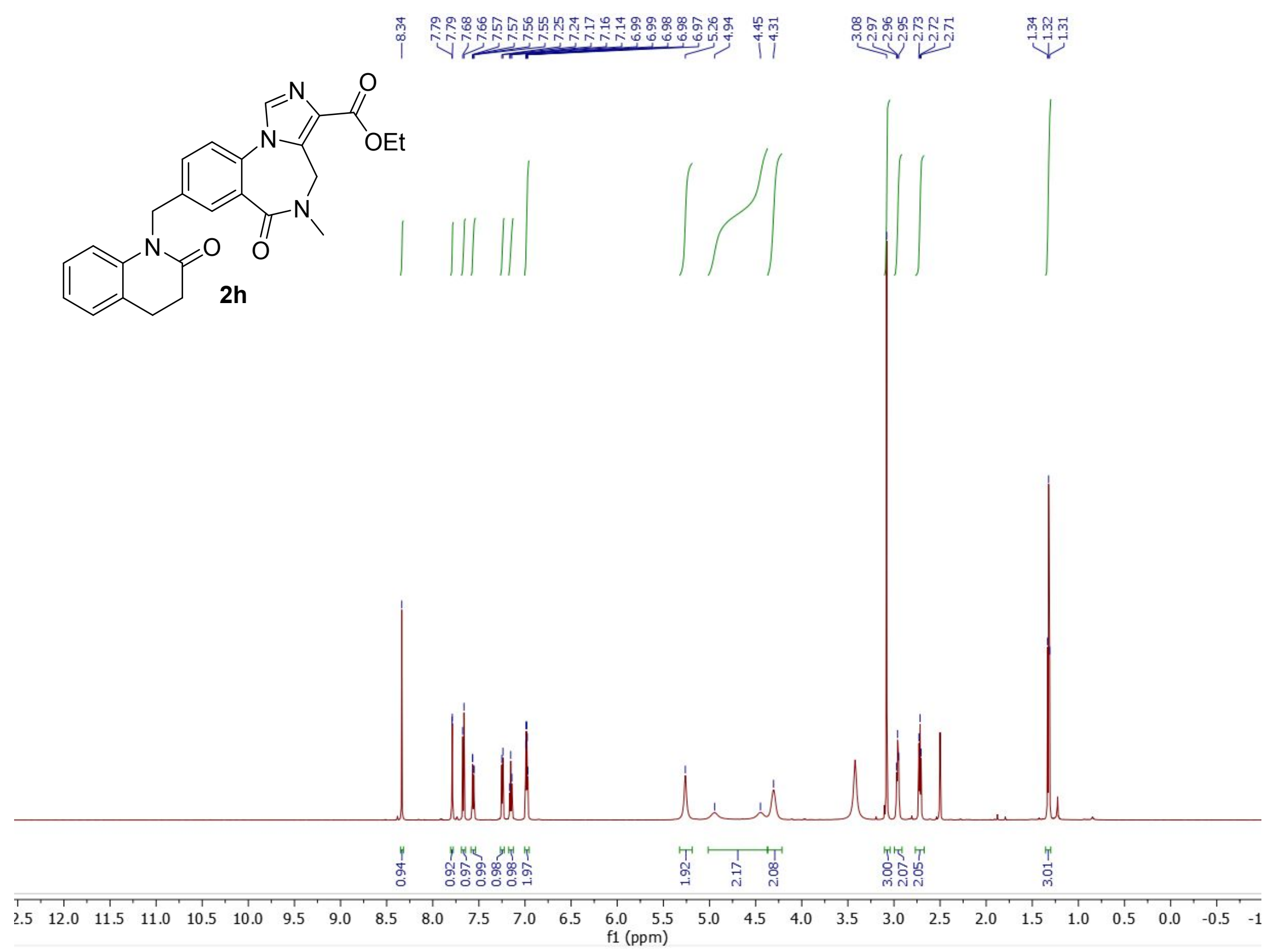




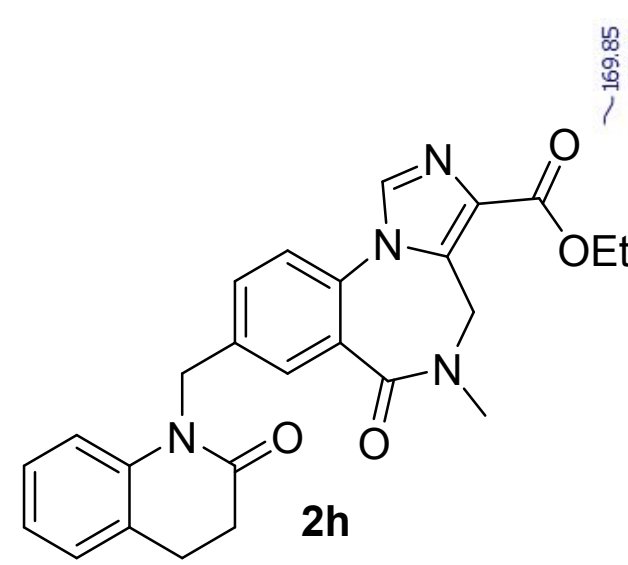

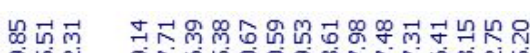

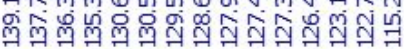

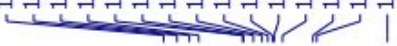

$2 \mathrm{~h}$
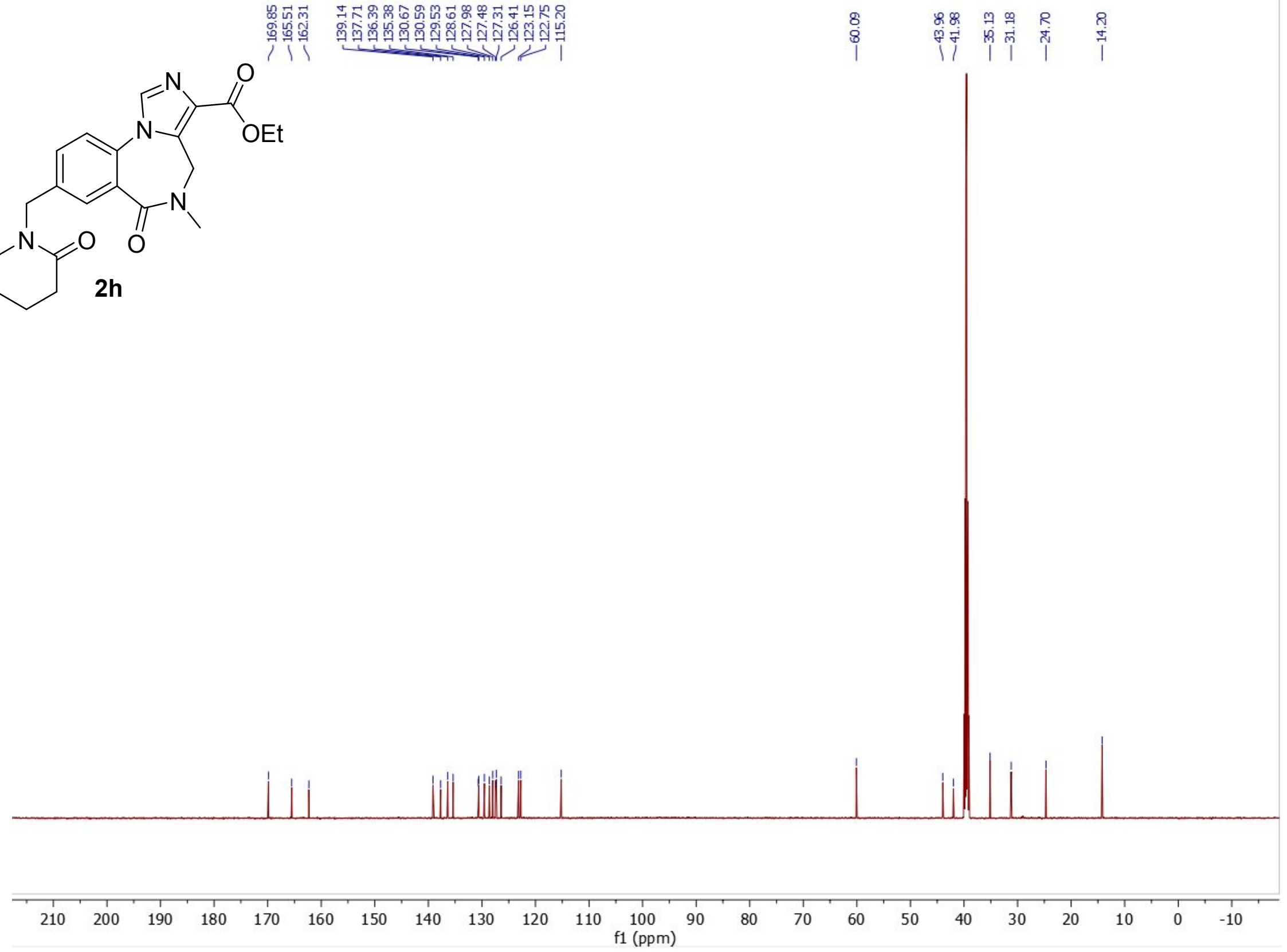


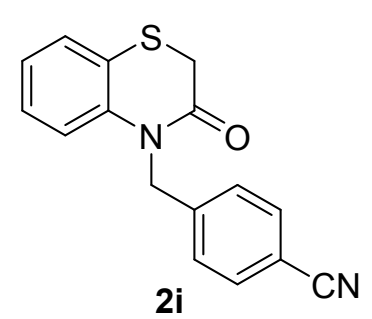

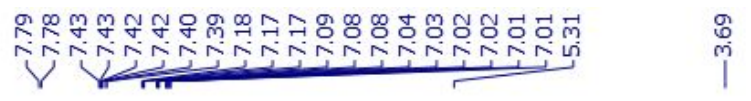
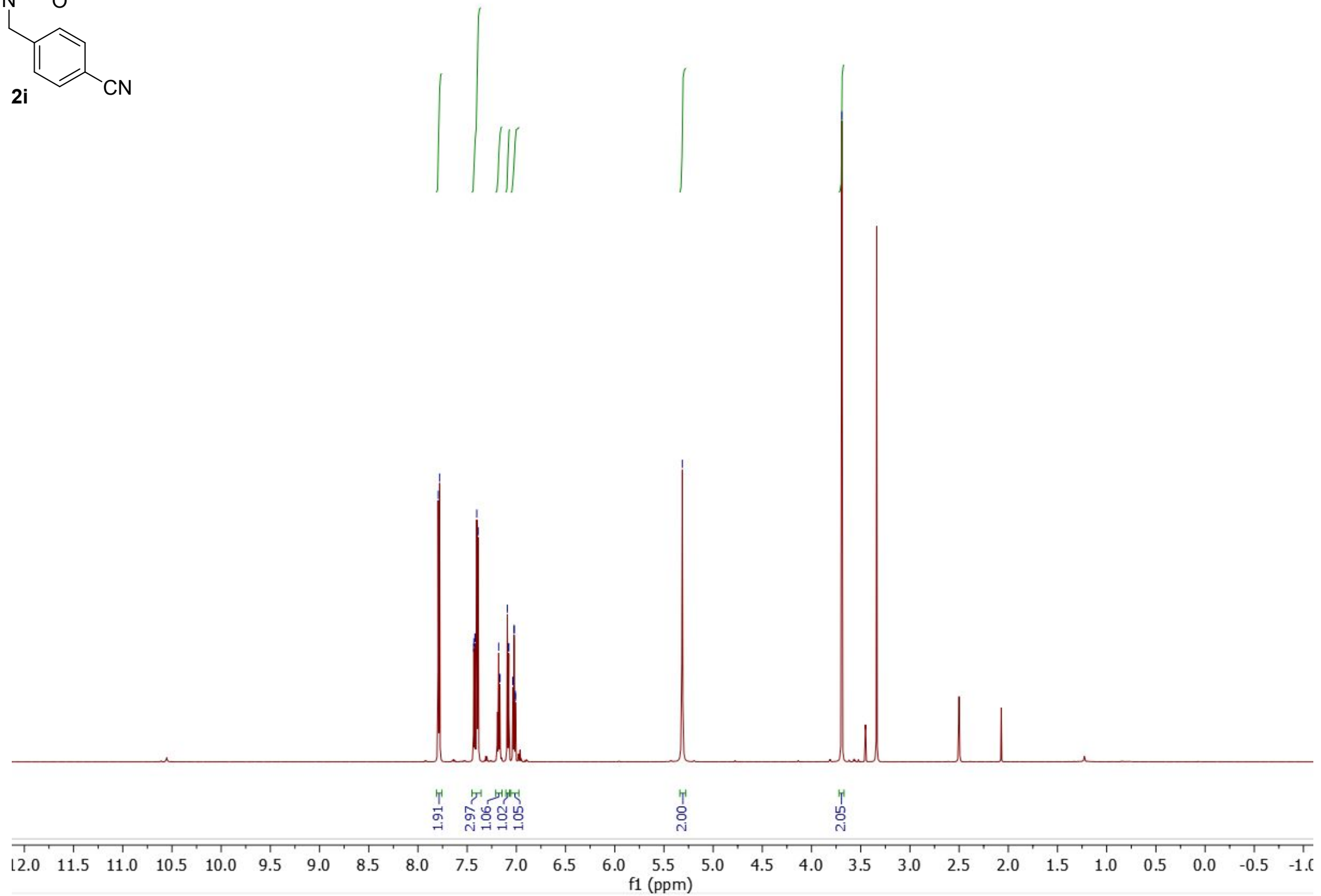


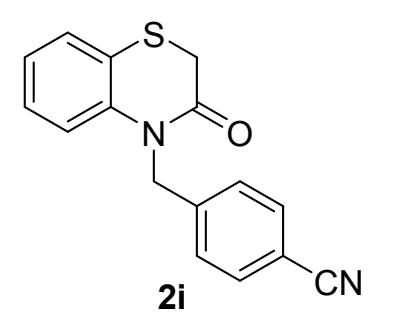

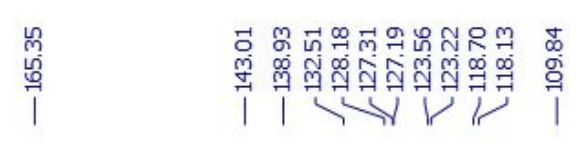

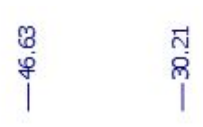

$2 i$

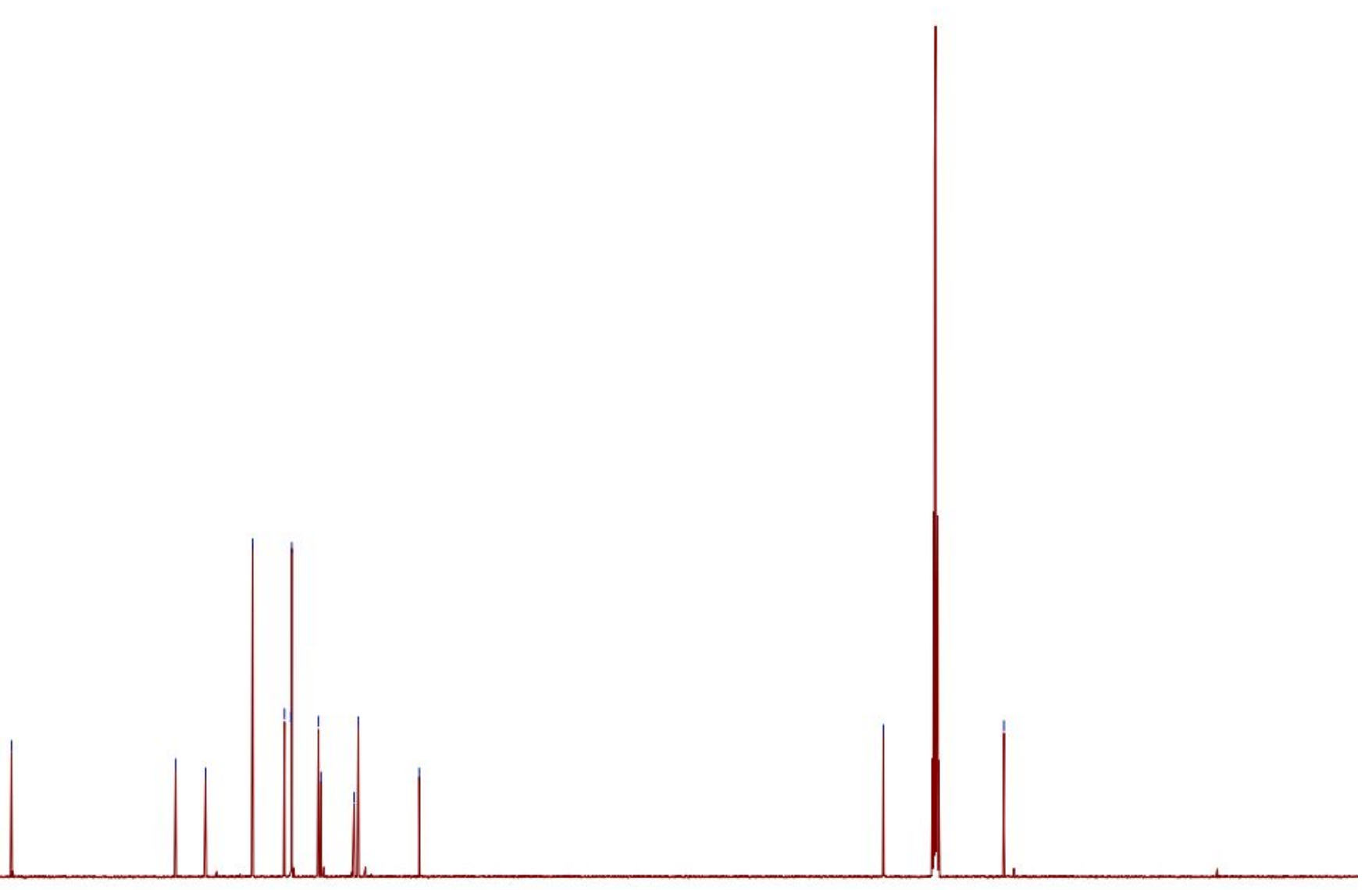

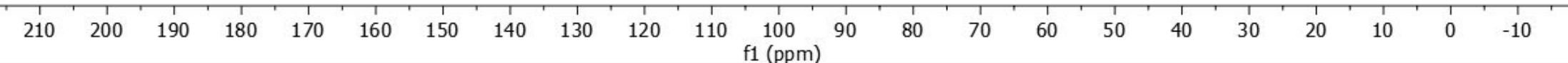



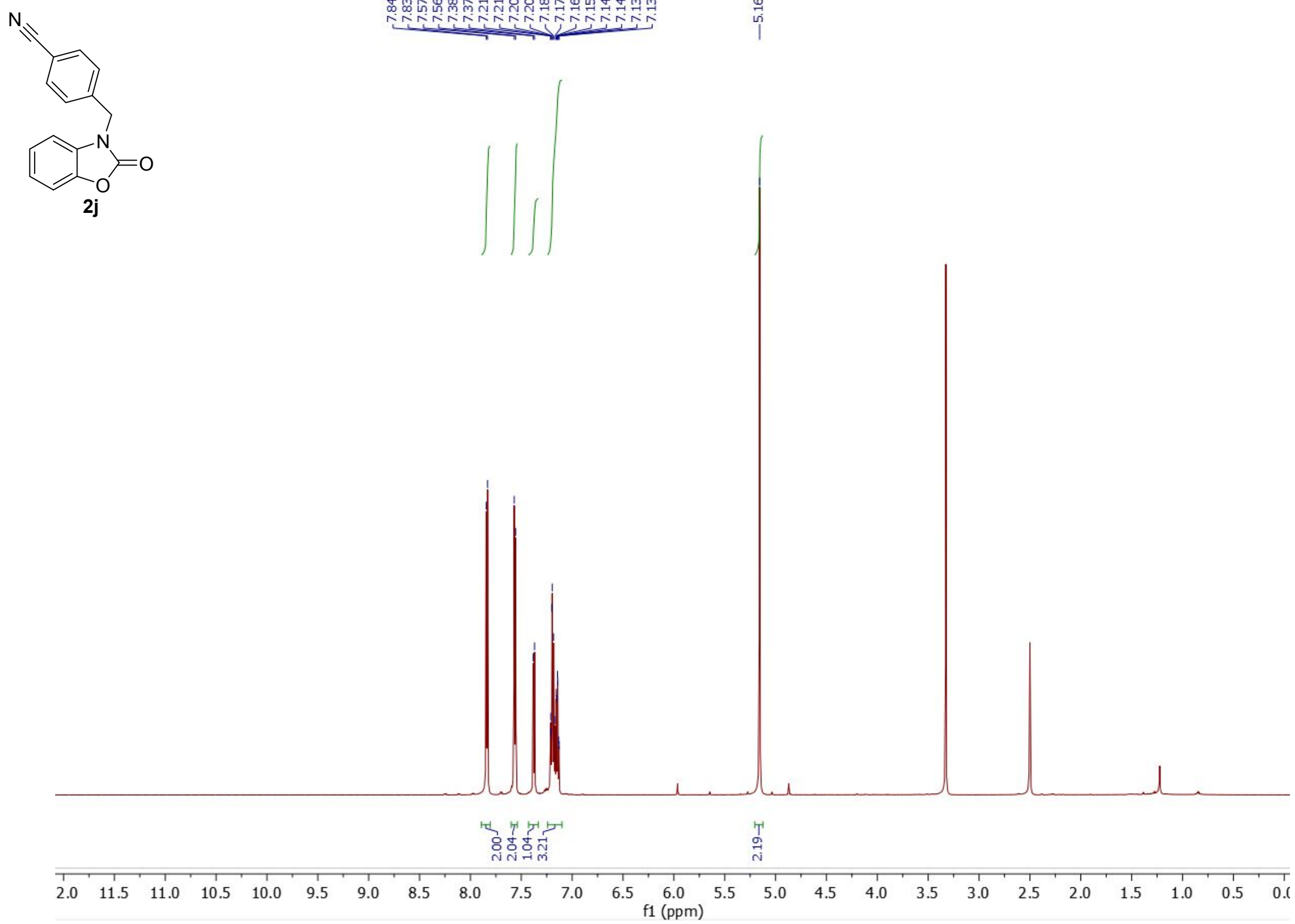

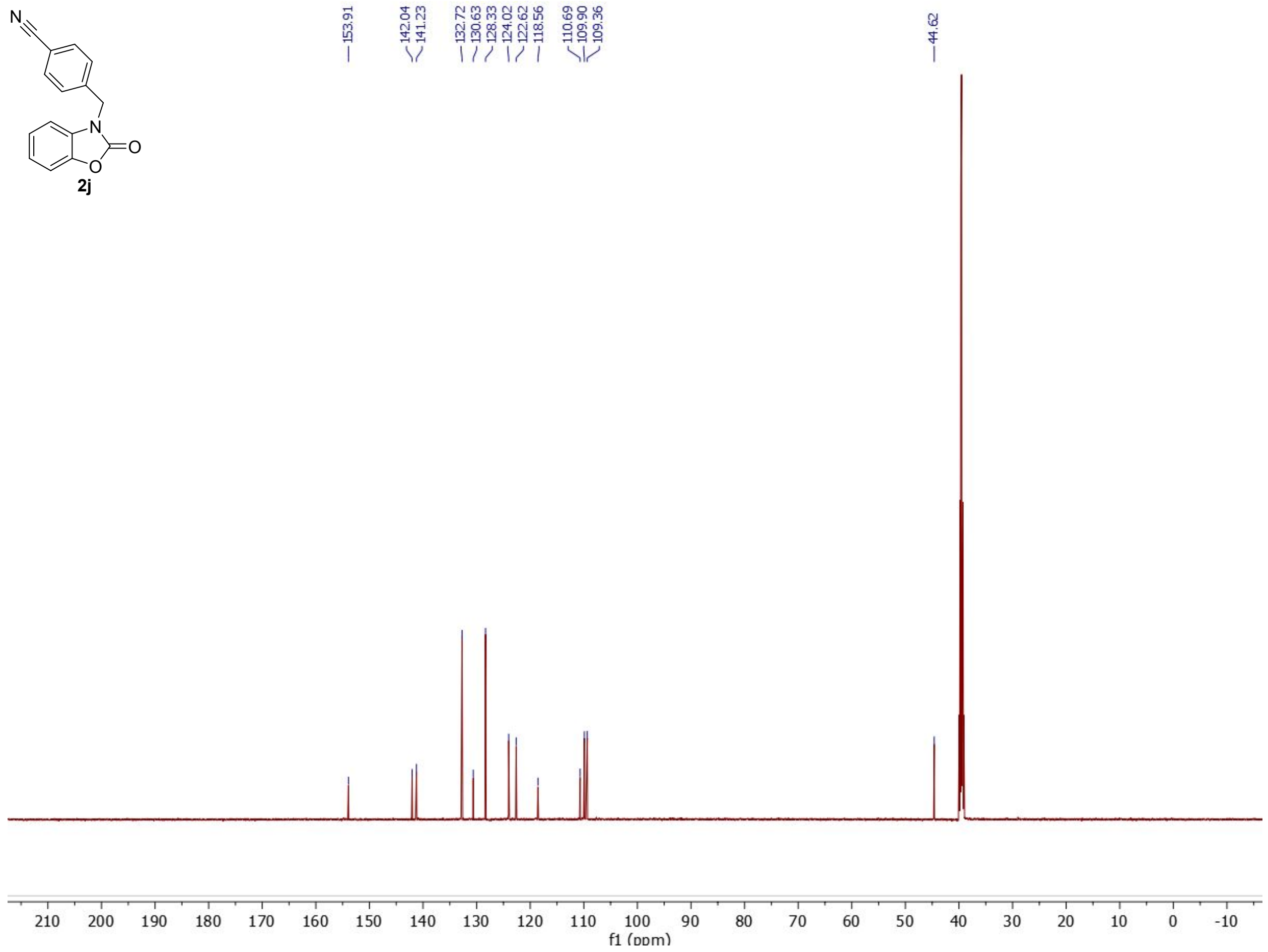

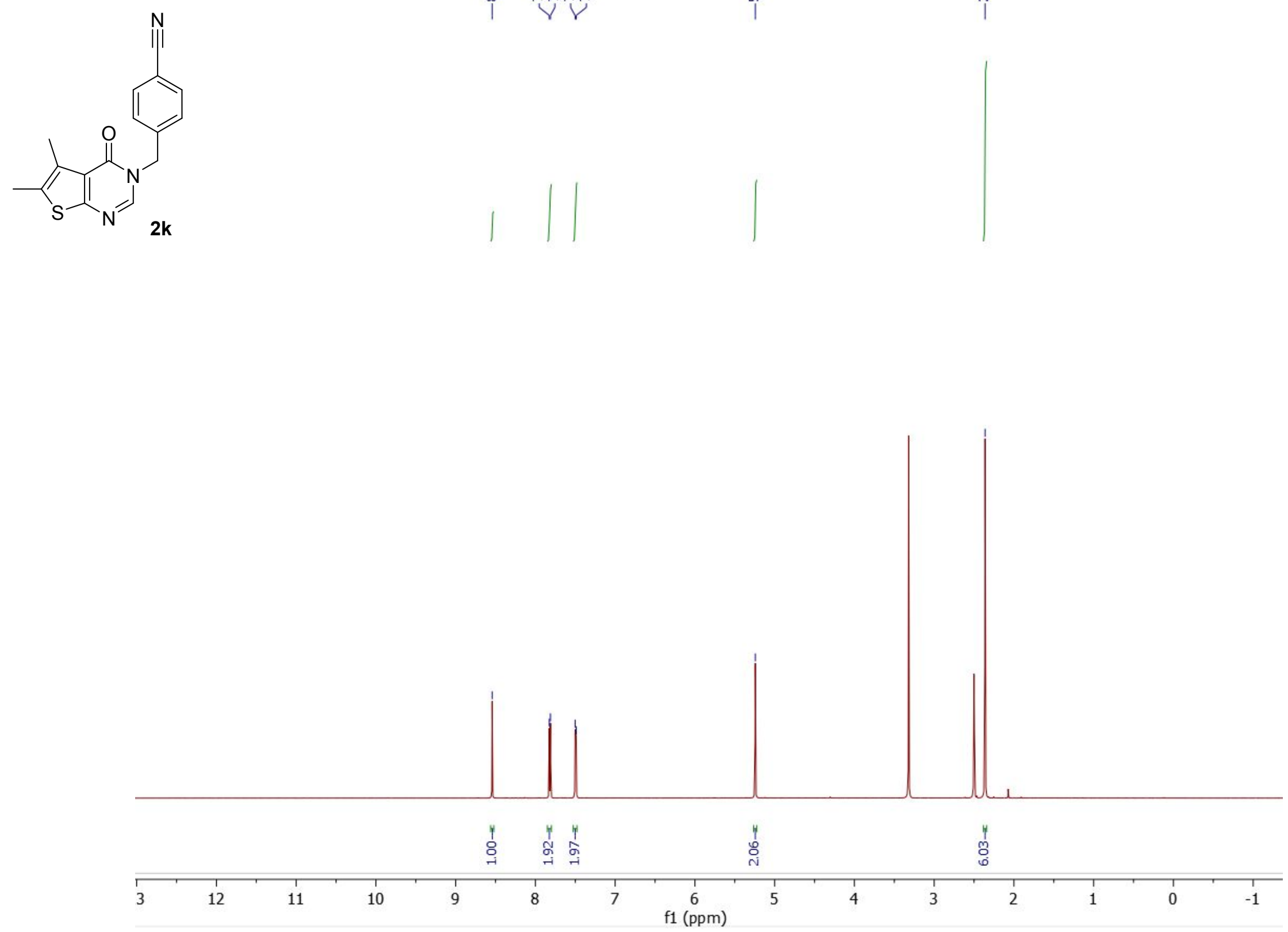


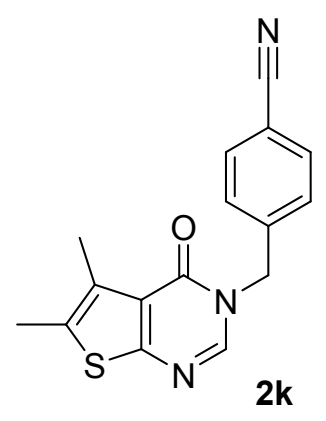

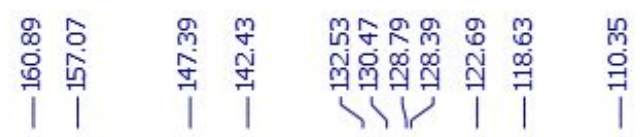

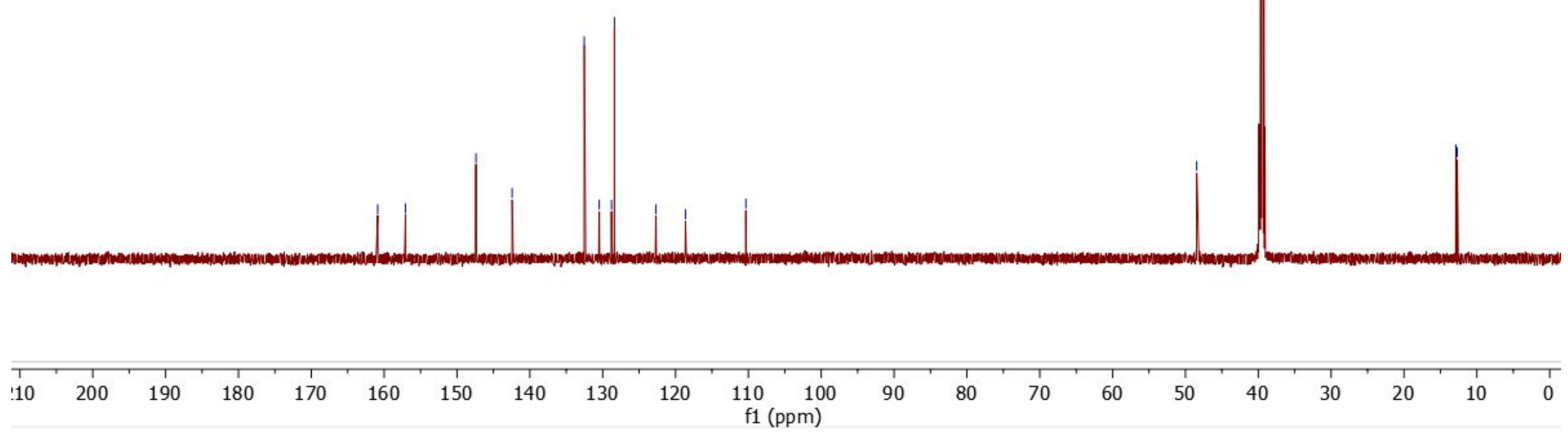




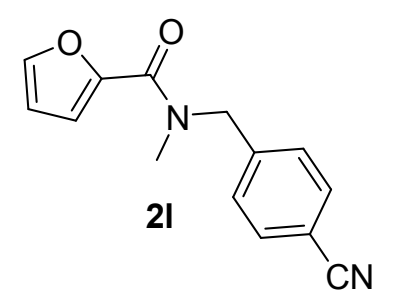

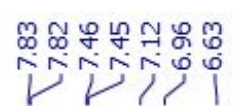

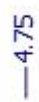

$\stackrel{9}{9}$
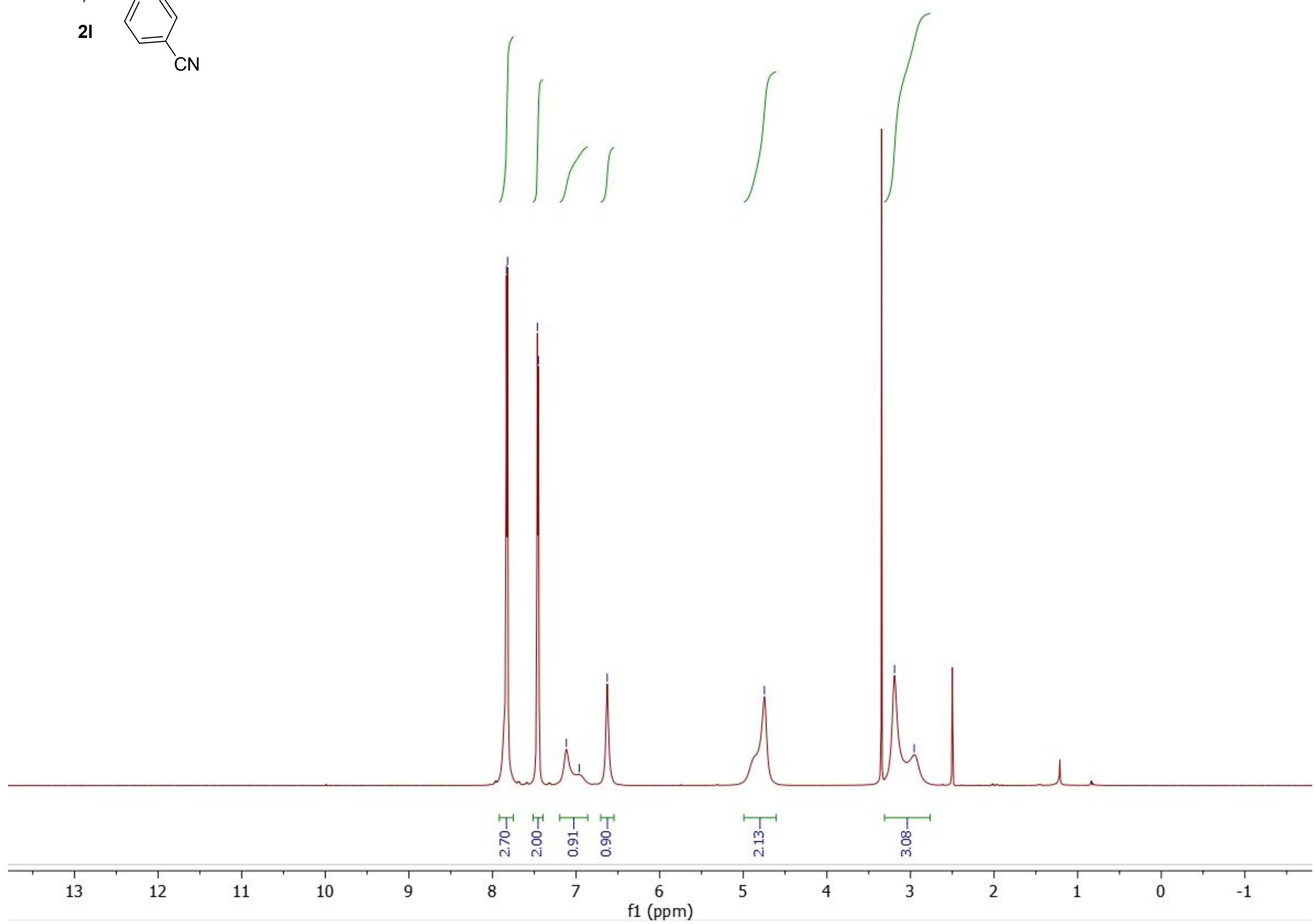

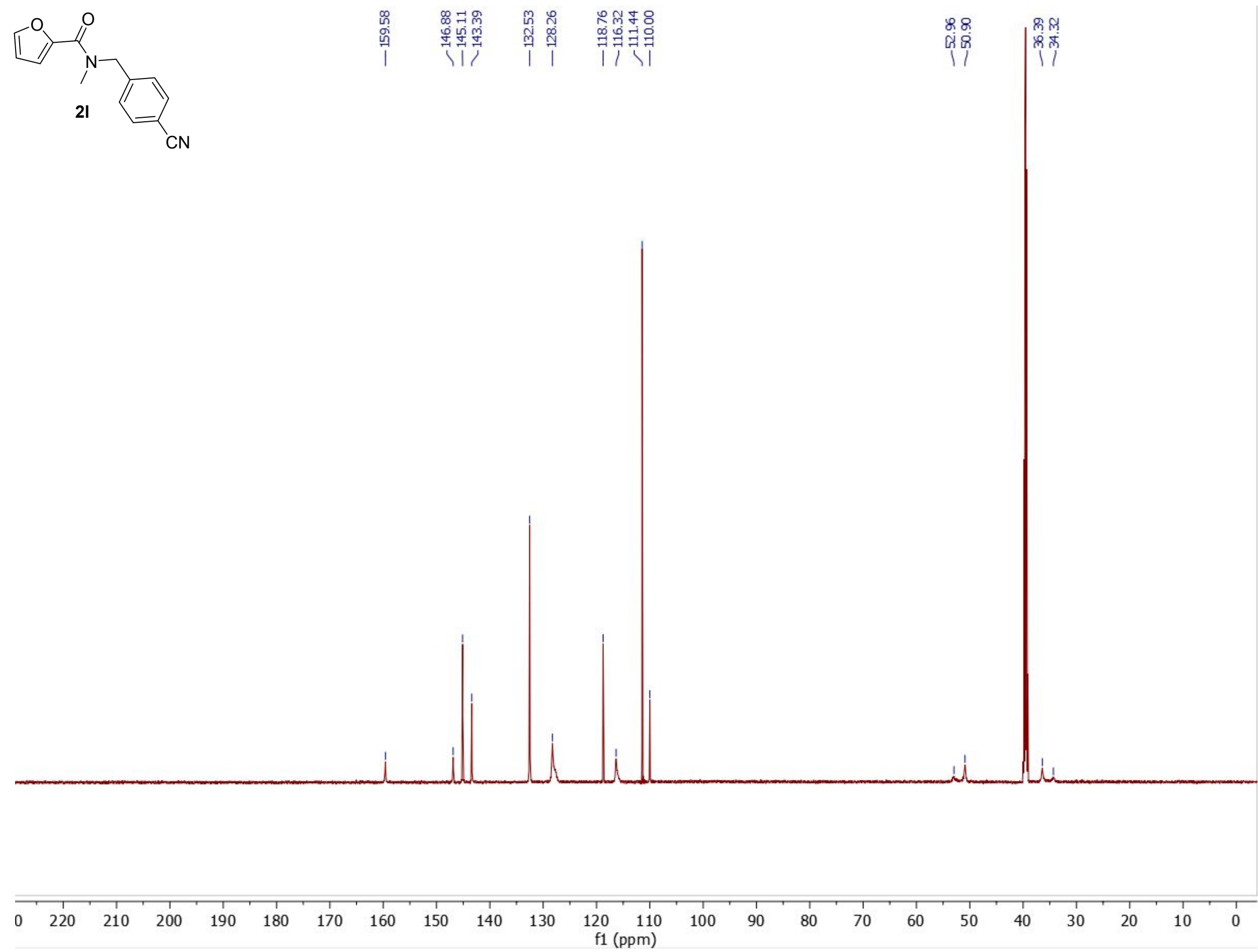


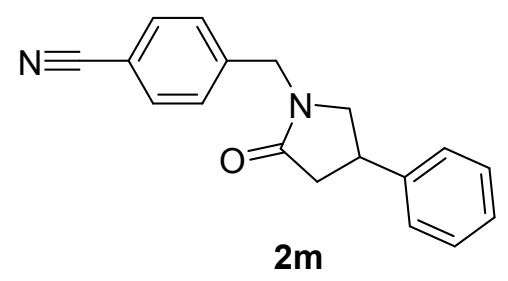

$2 m$

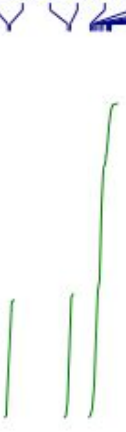

प

$|1|$
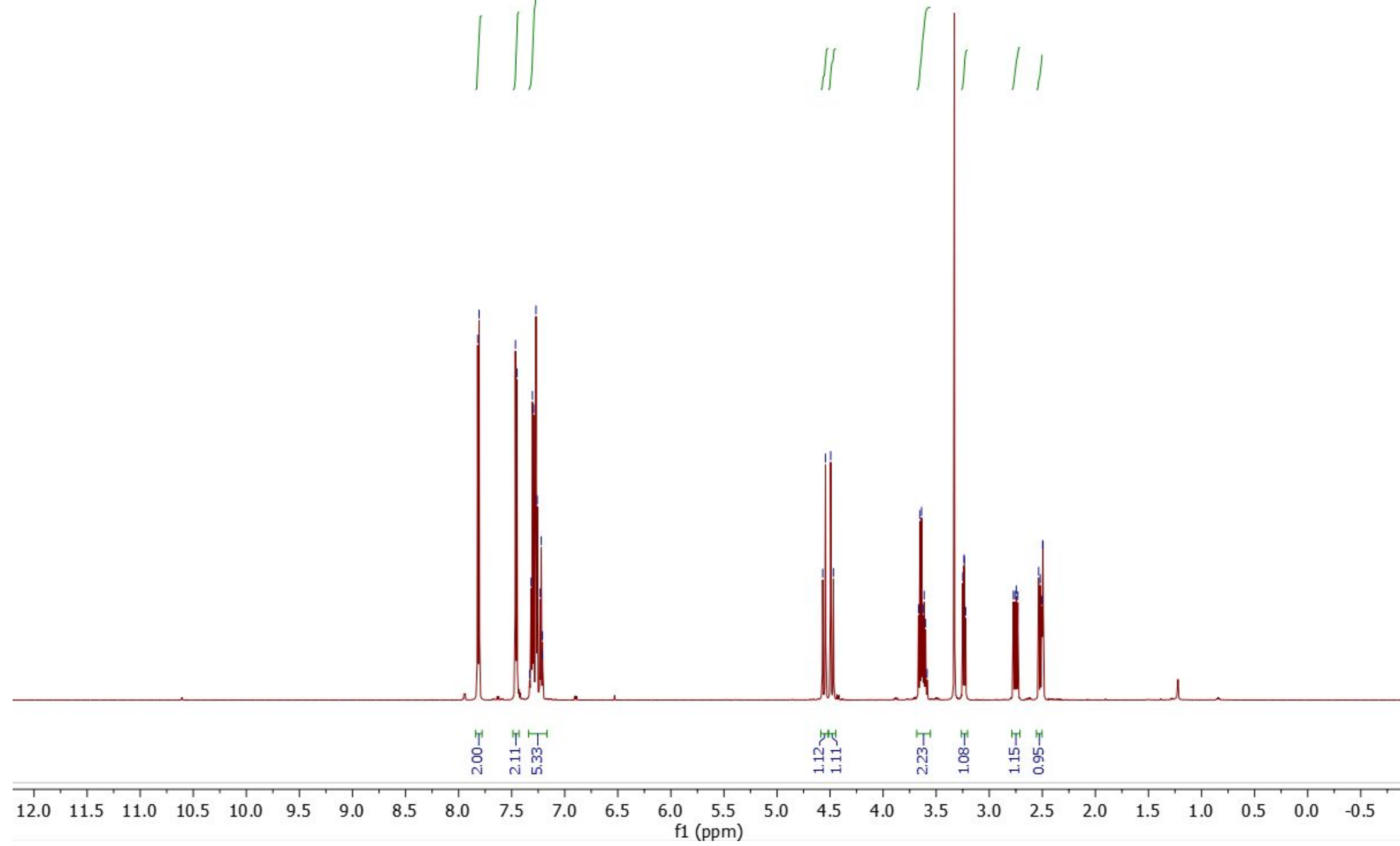

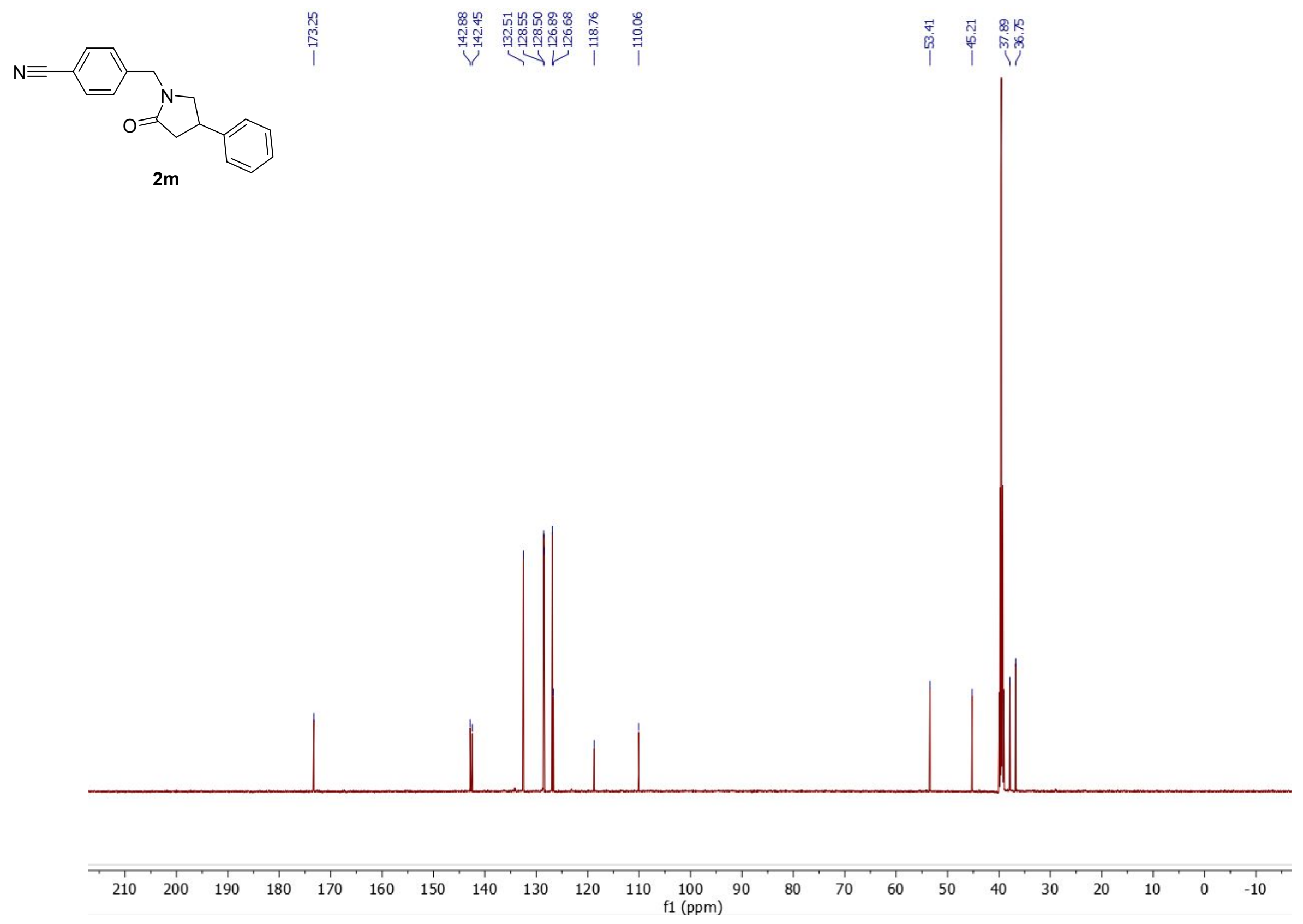

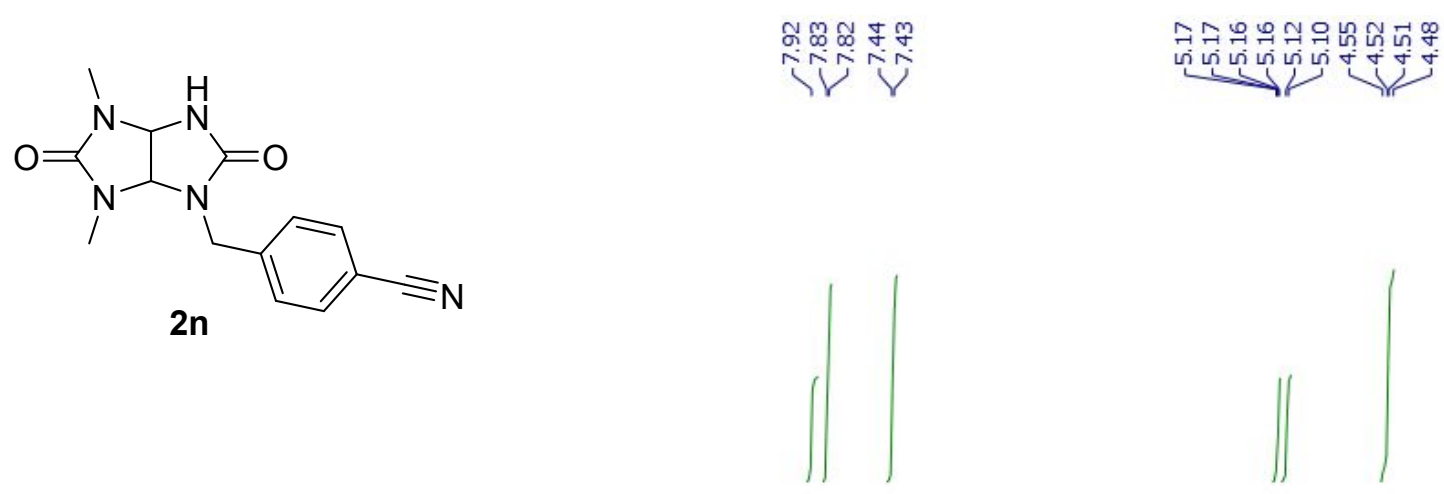

$2 n$
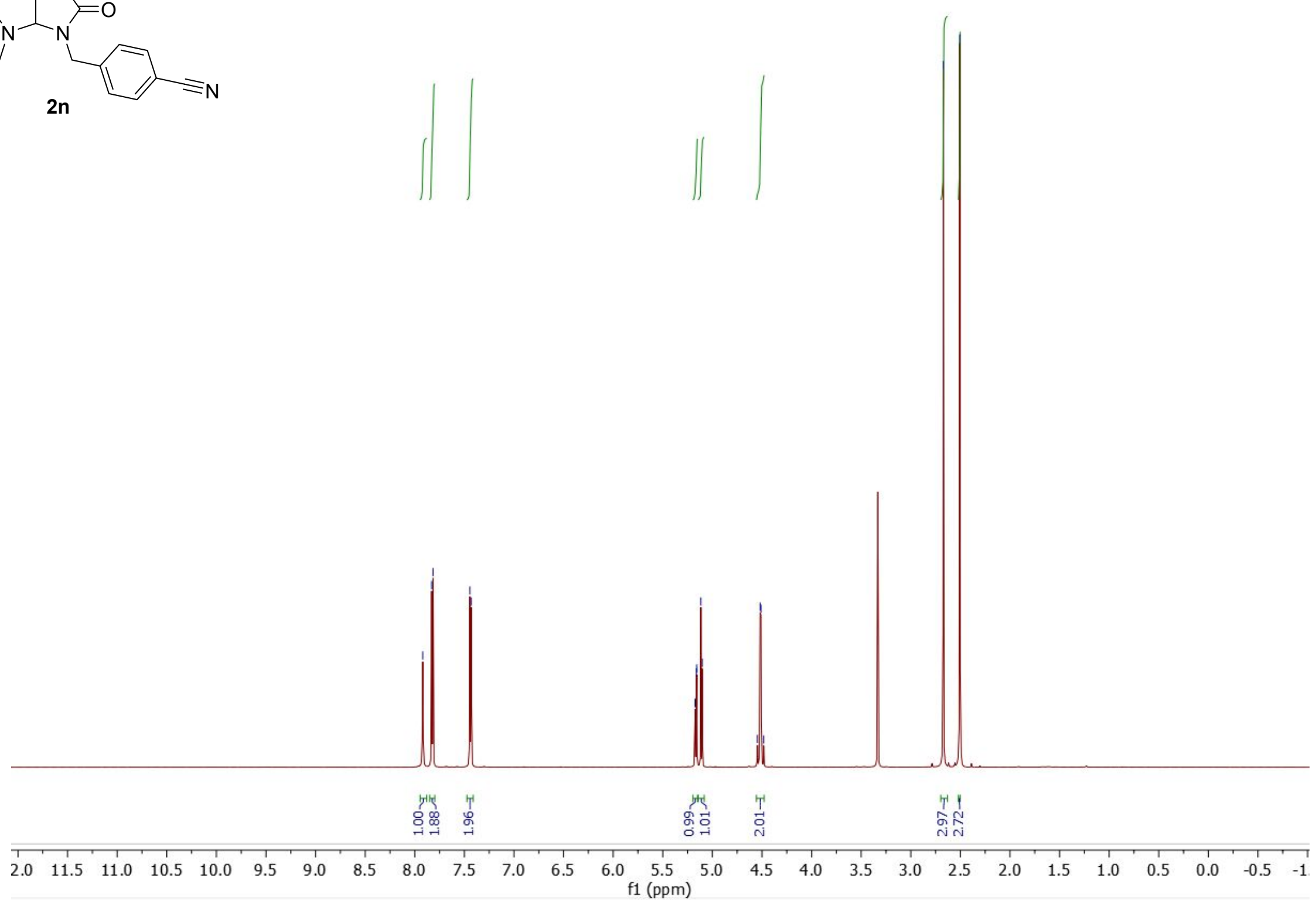


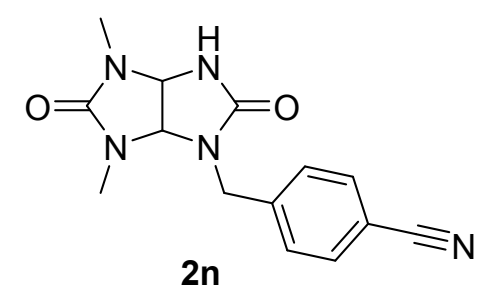

I

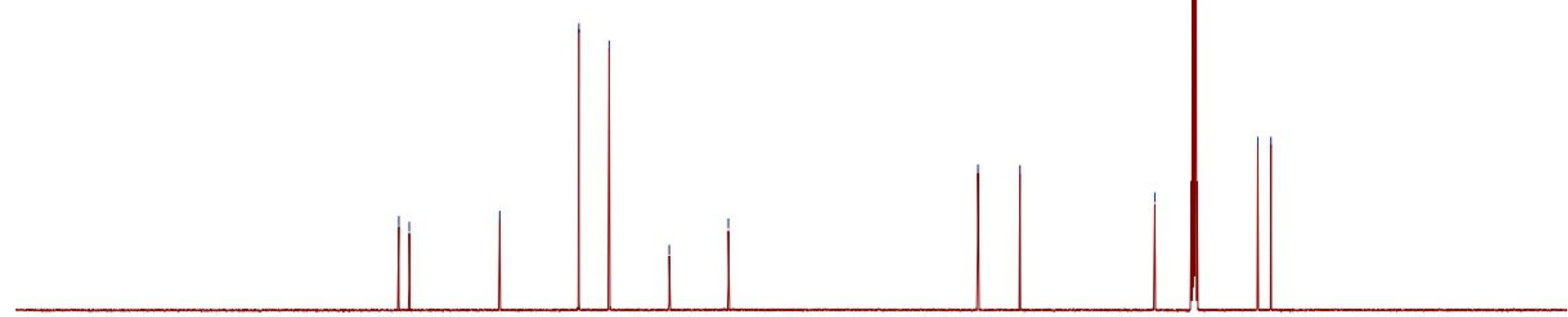




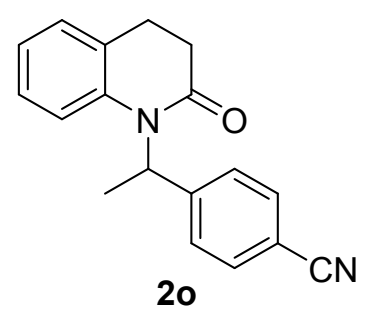

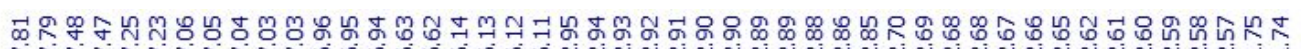

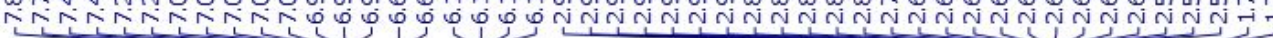
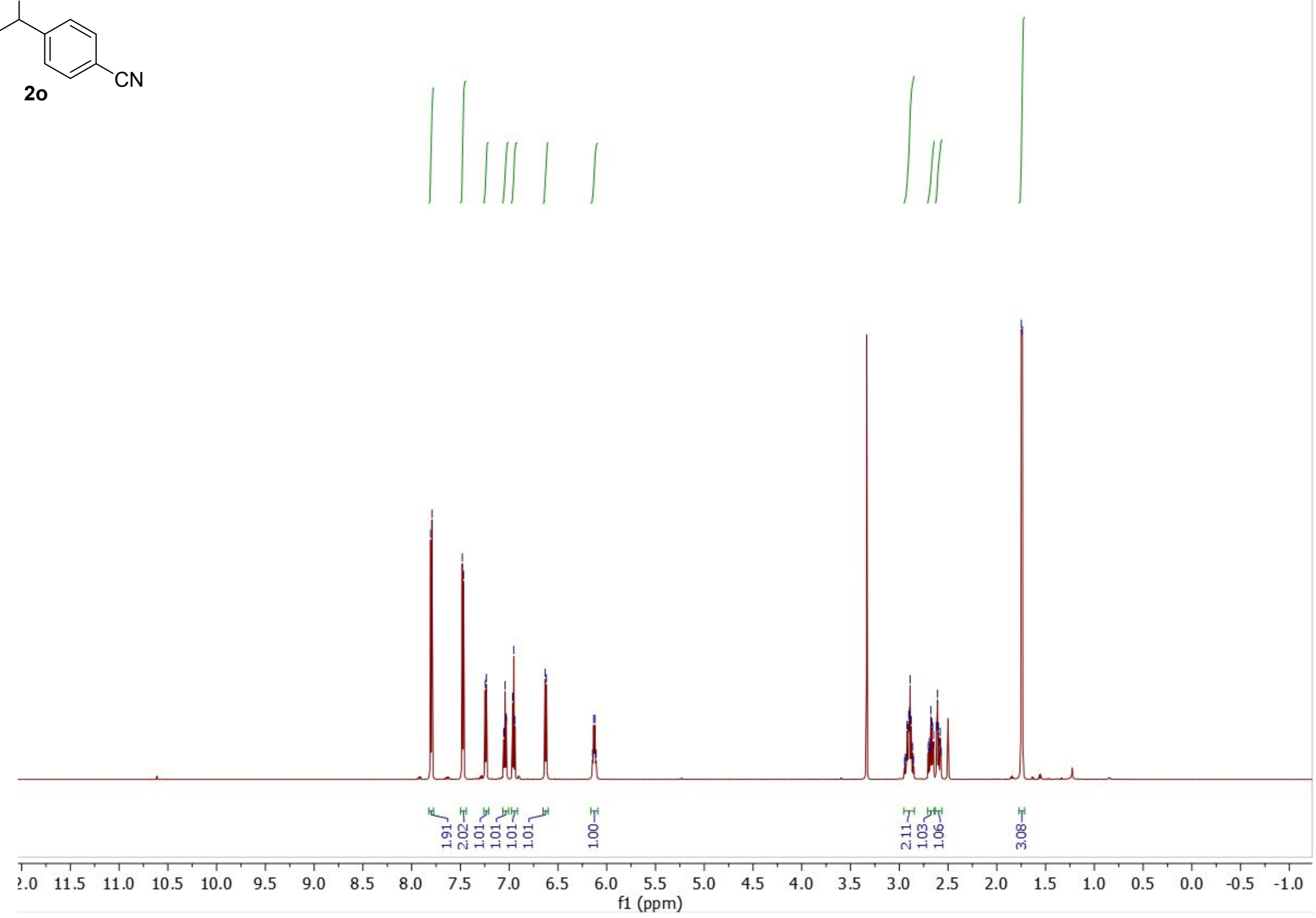


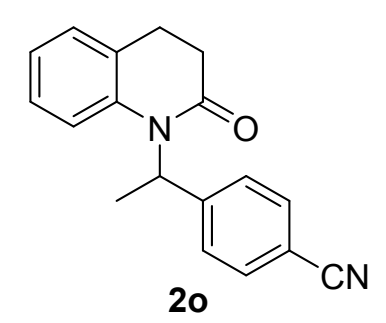

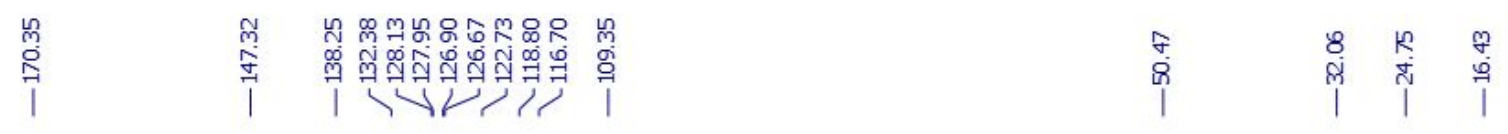

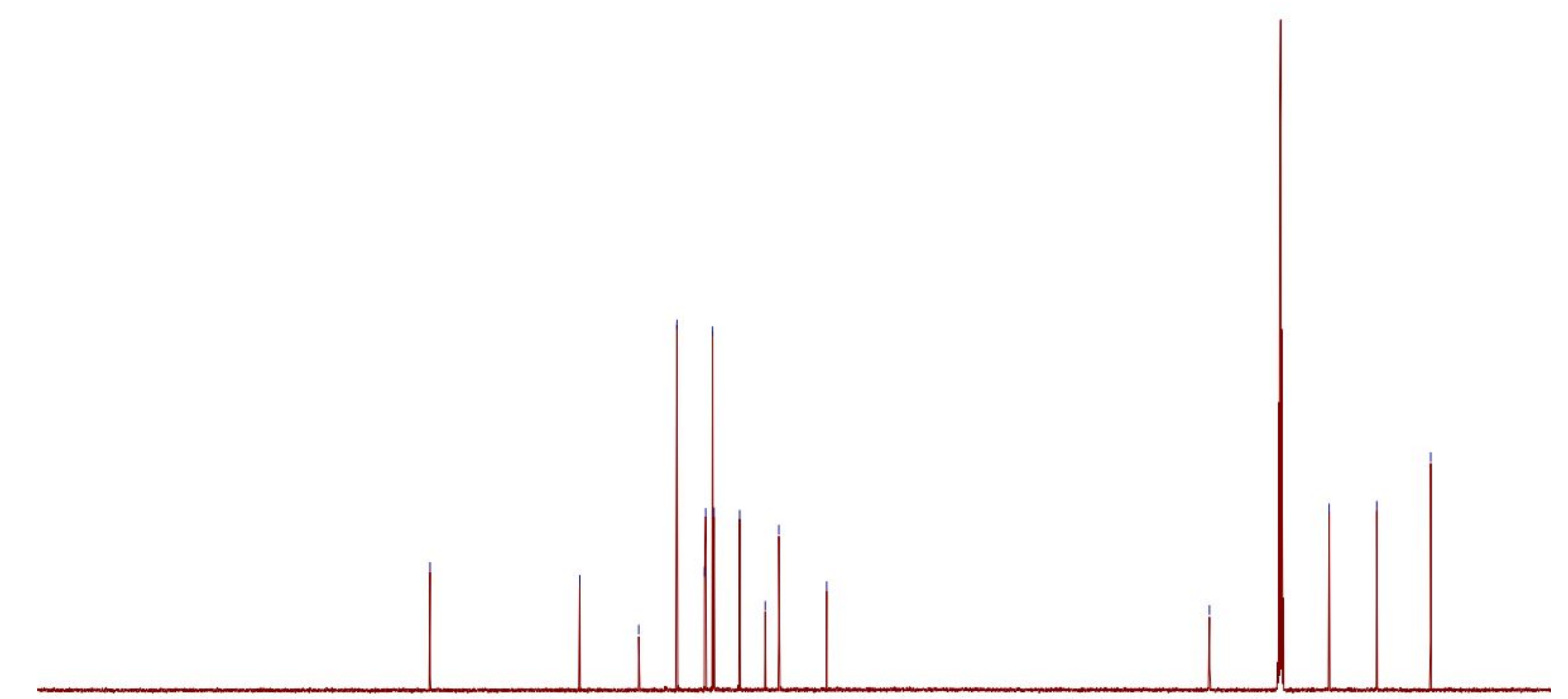

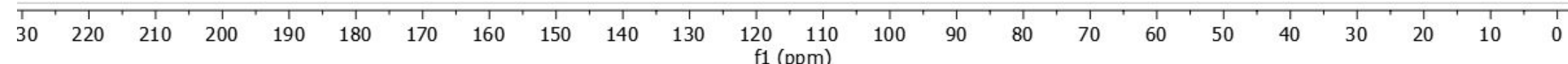




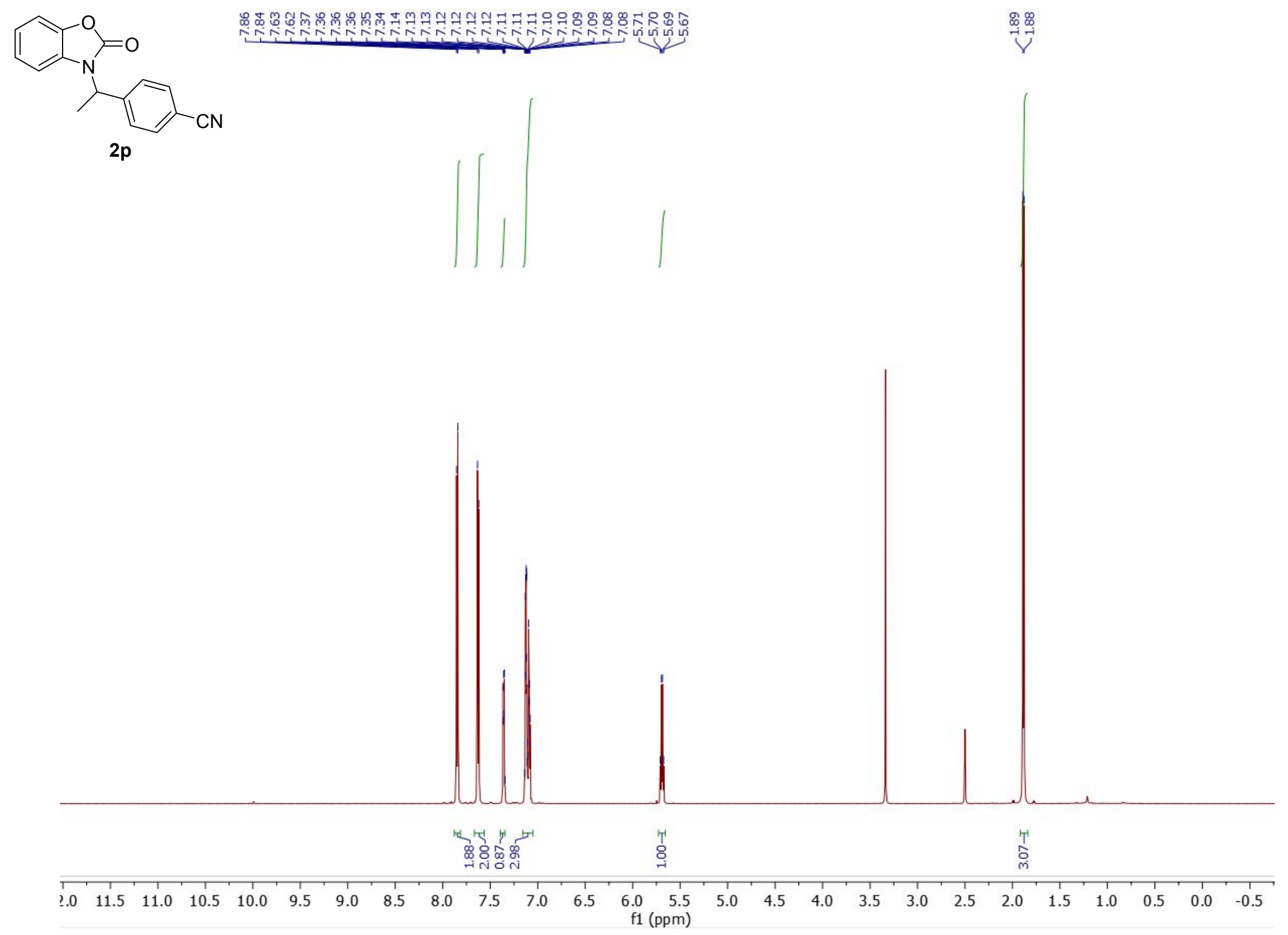



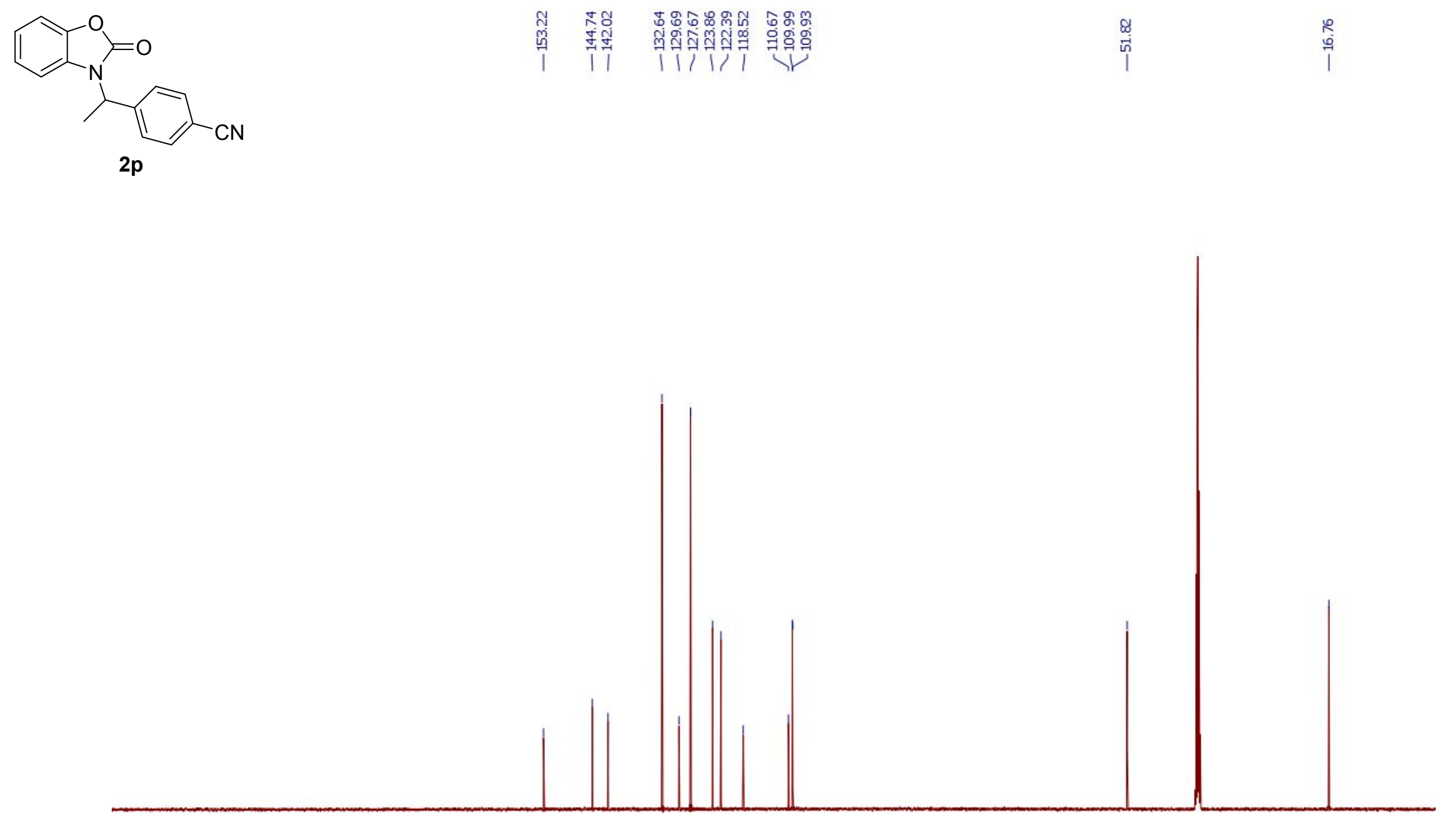

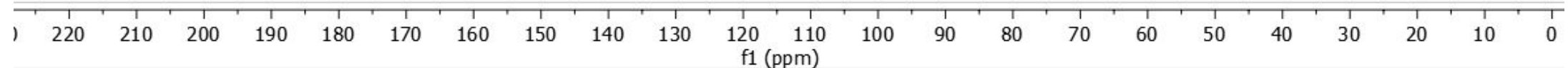



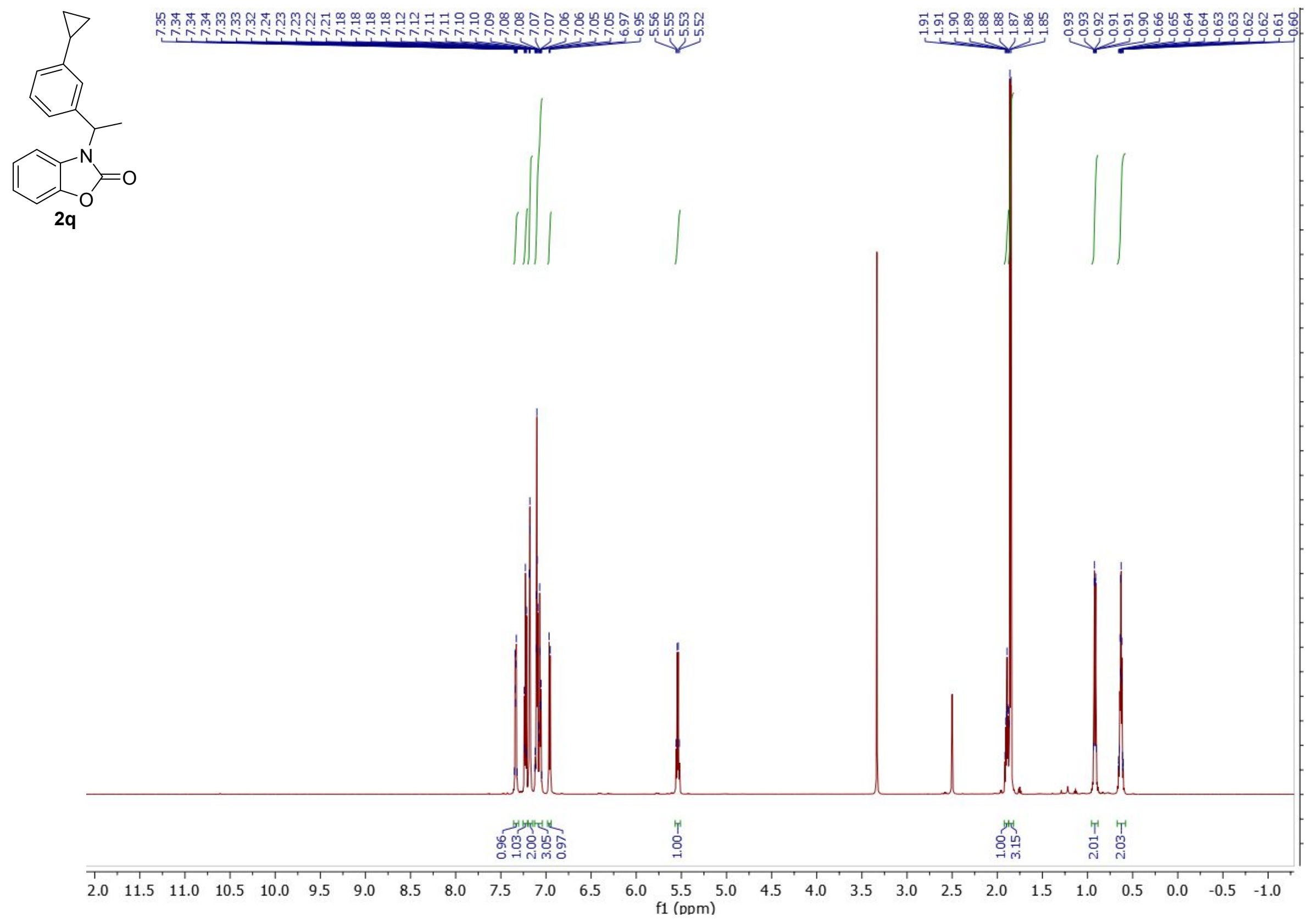

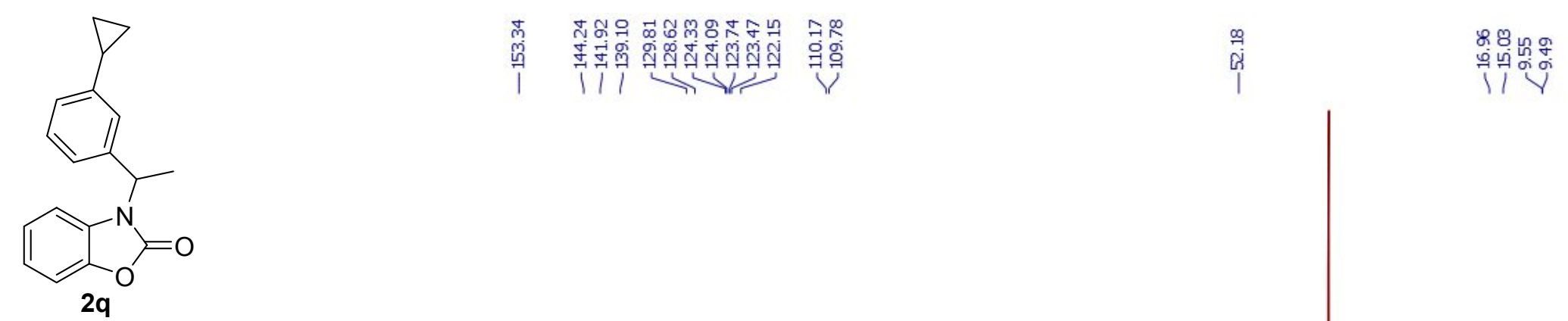


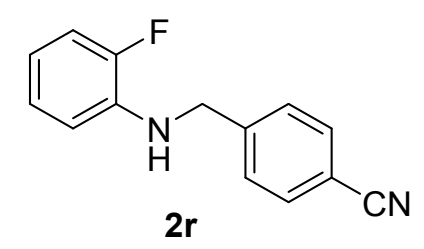

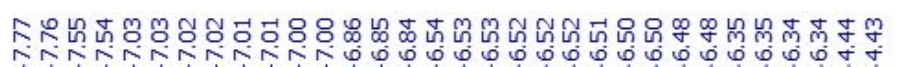
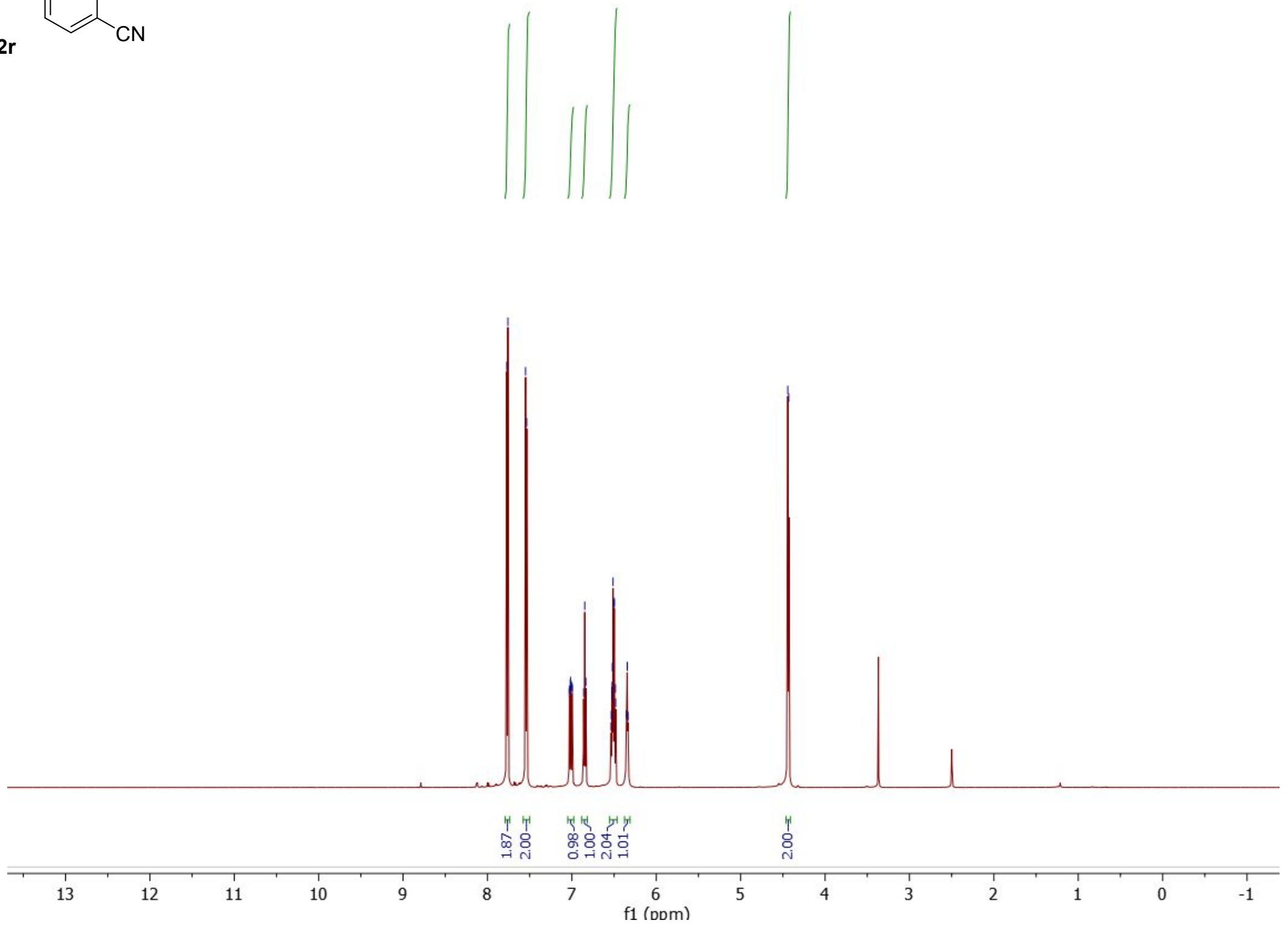


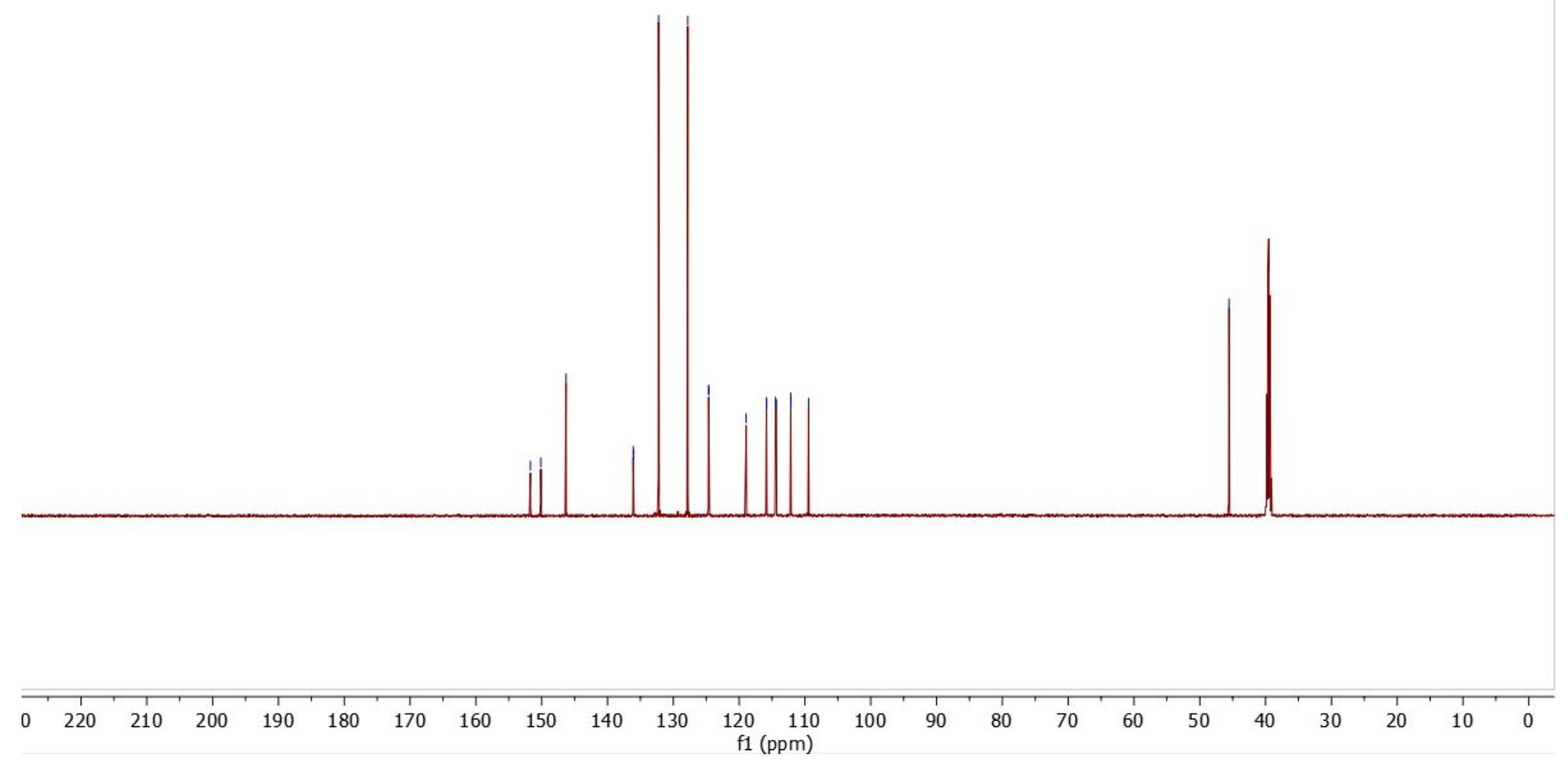



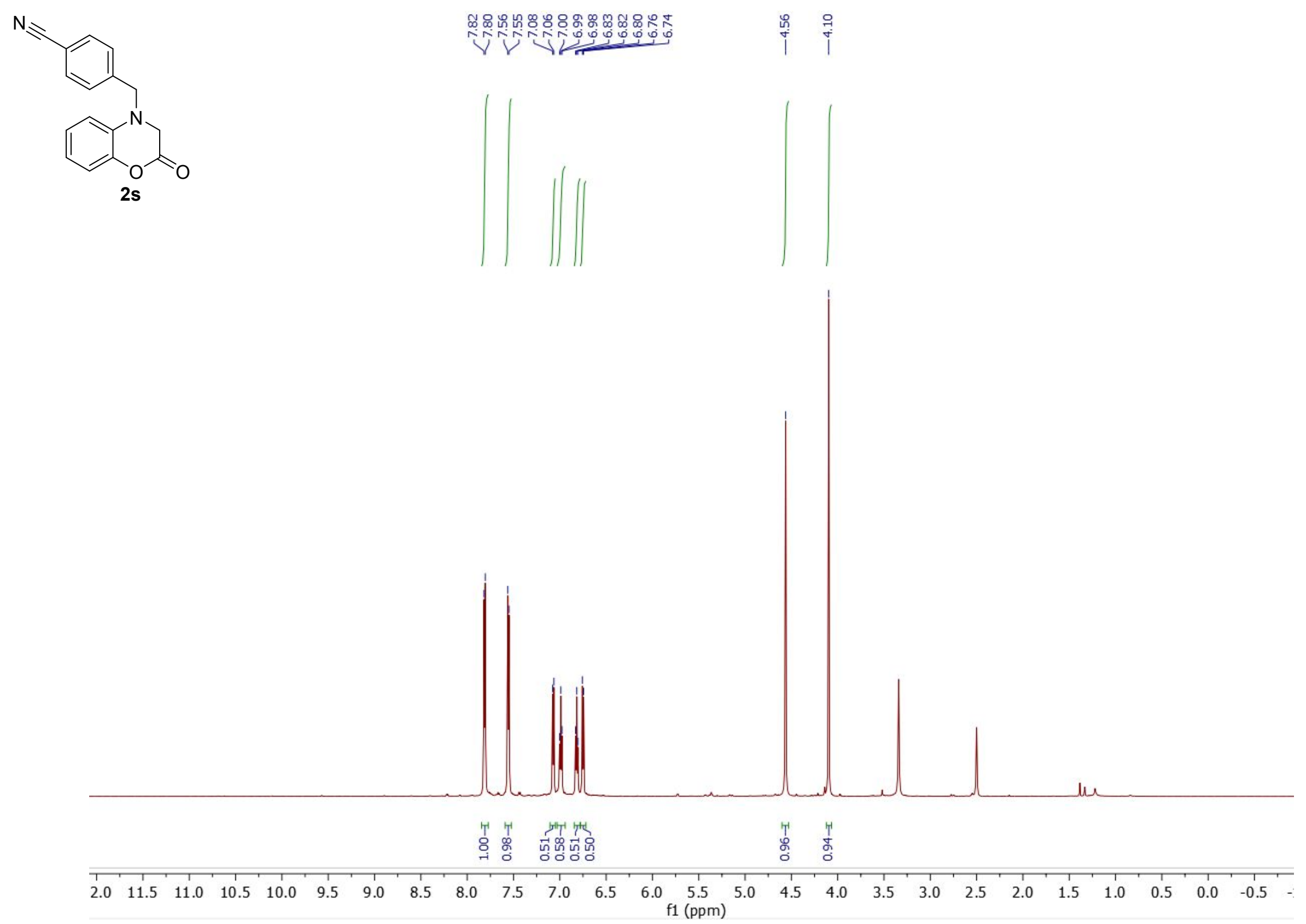


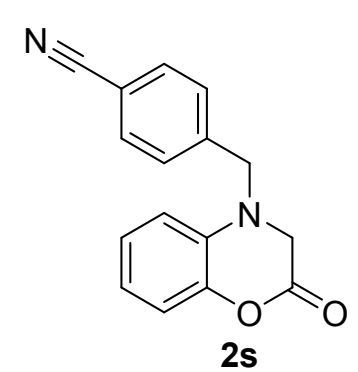

2s

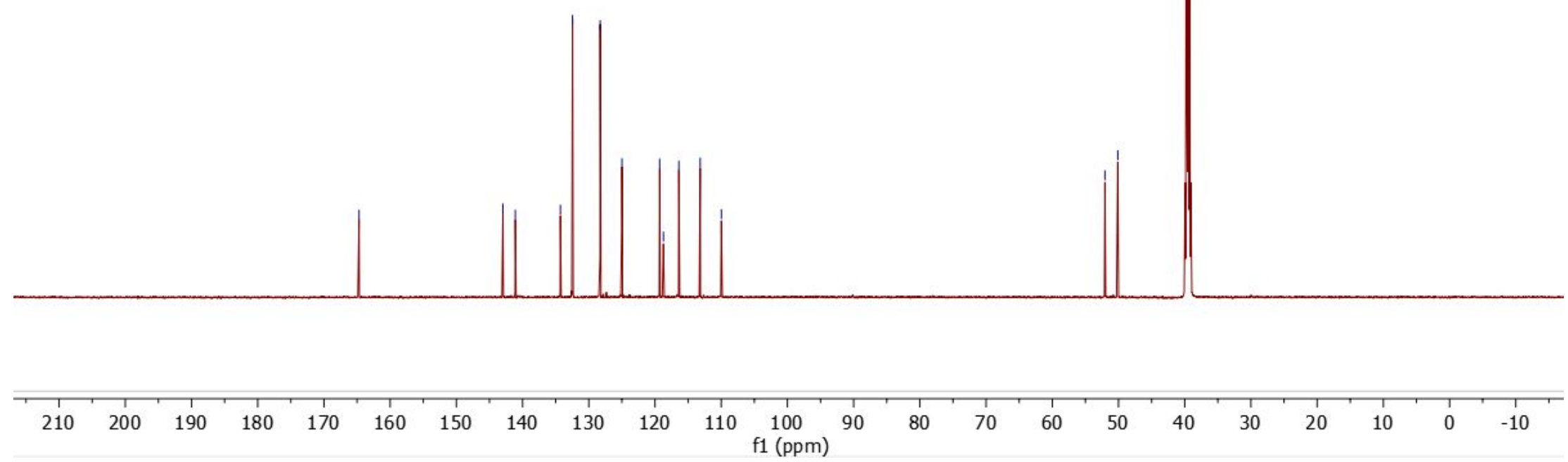




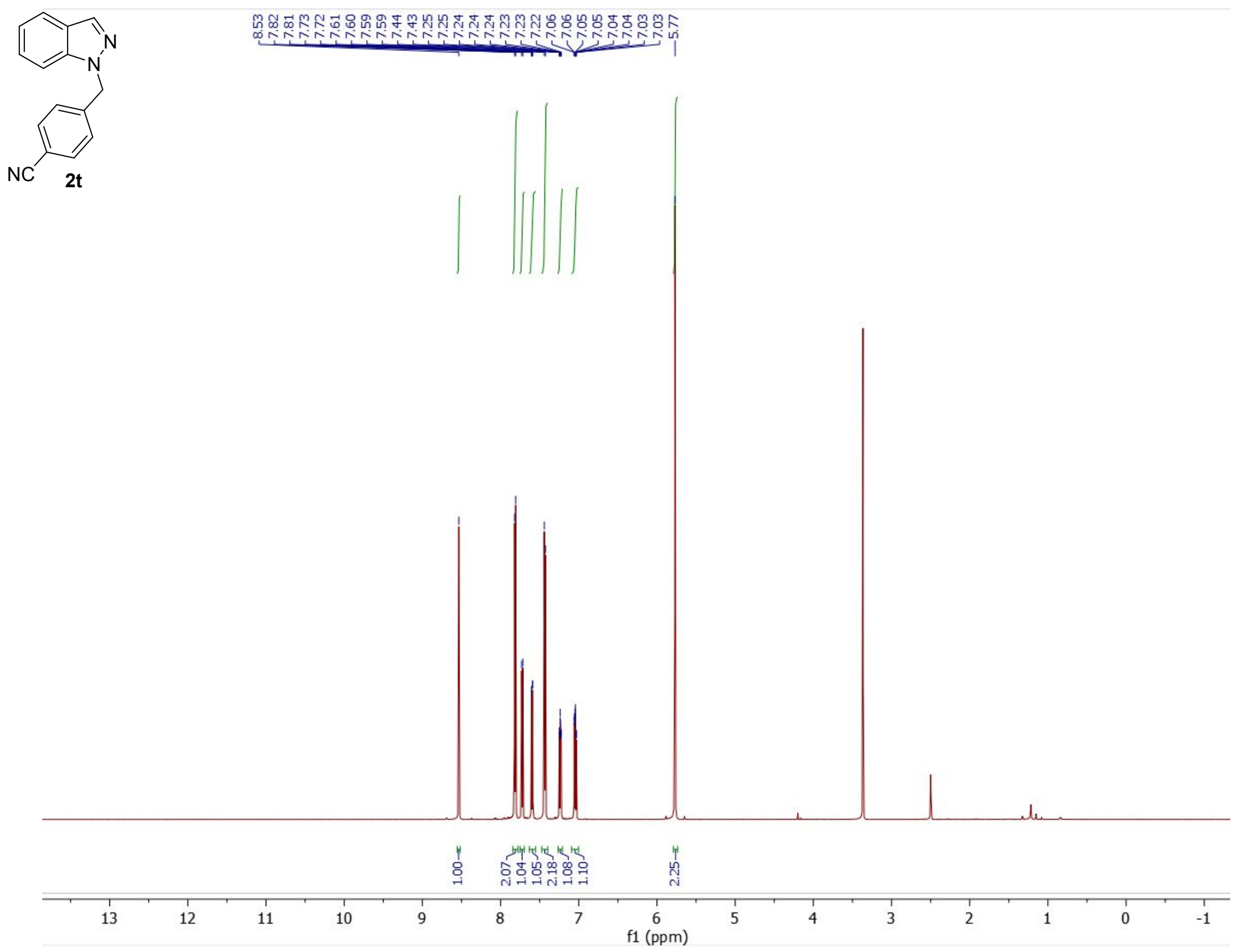




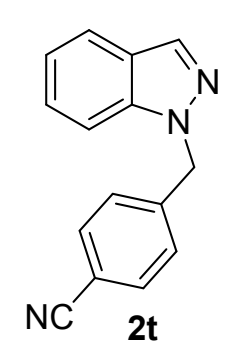




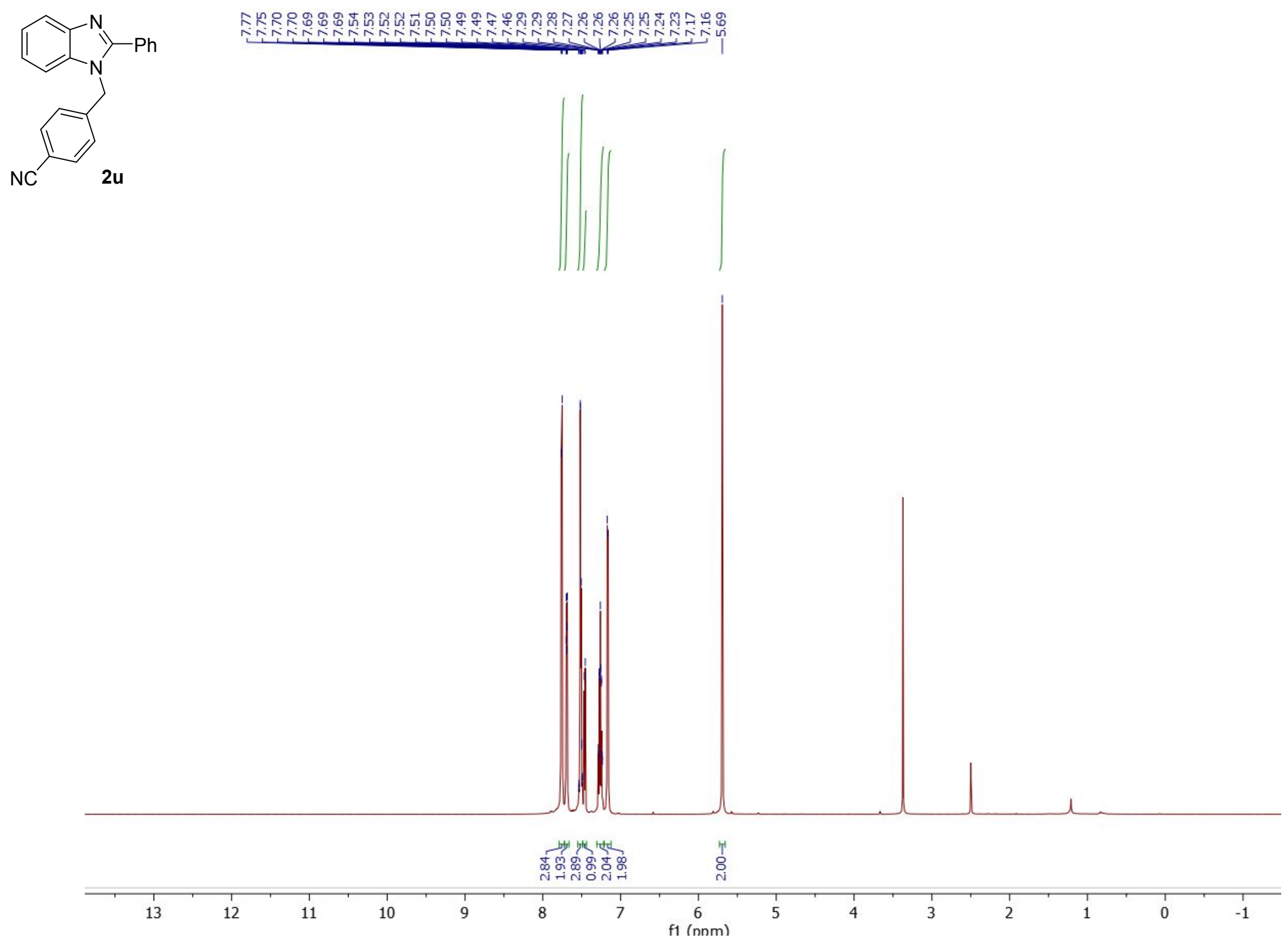



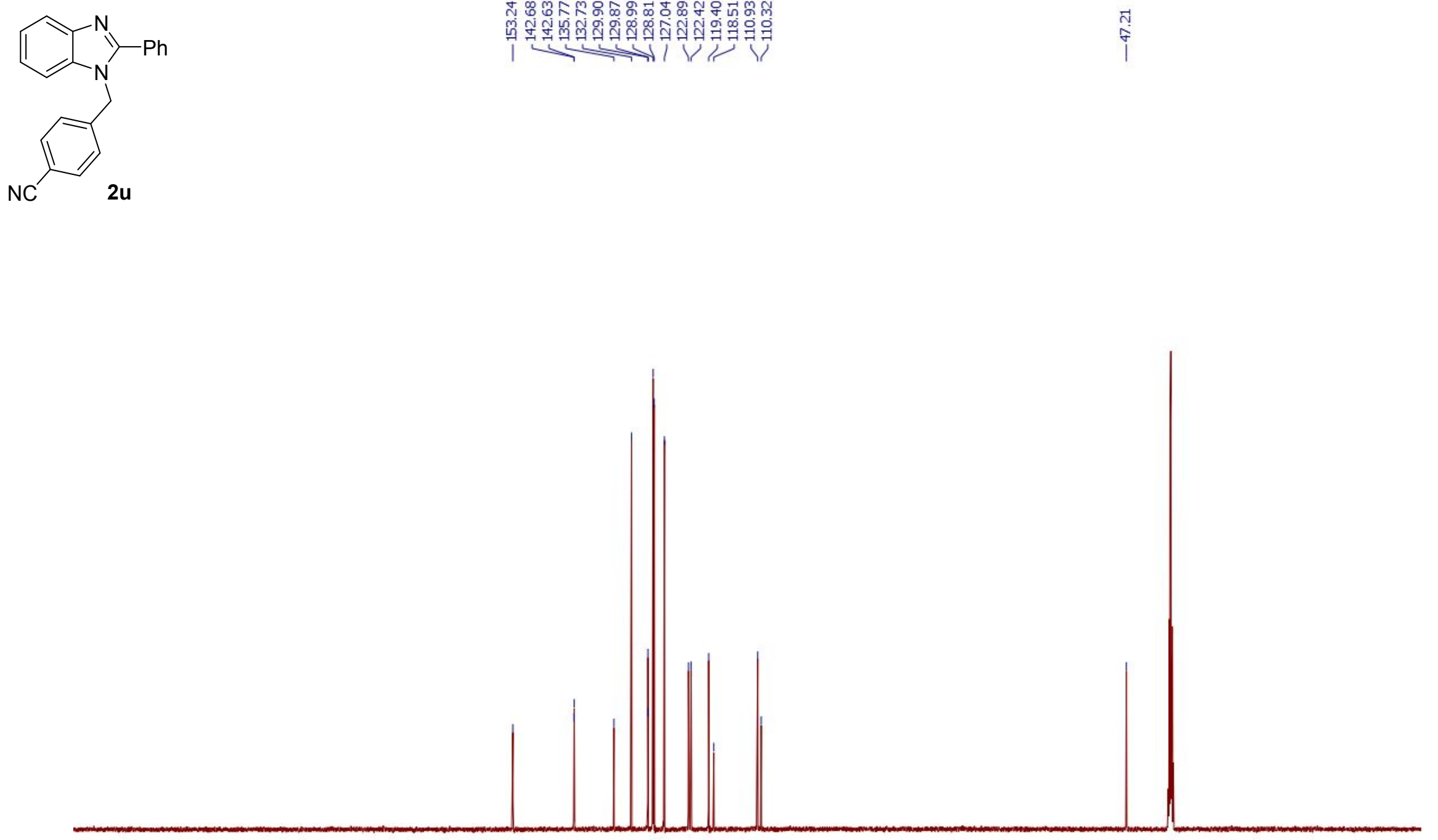

\begin{tabular}{|c|c|c|c|c|c|c|c|c|c|c|c|c|c|c|c|c|c|c|c|c|c|}
\hline 220 & 210 & 200 & $\begin{array}{c}1 \\
190\end{array}$ & $\begin{array}{c}1 \\
180\end{array}$ & $\begin{array}{l}1 \\
170\end{array}$ & $\begin{array}{c}1 \\
160\end{array}$ & $\begin{array}{c}1 \\
150\end{array}$ & $\begin{array}{c}1 \\
140\end{array}$ & $\begin{array}{c}1 \\
130\end{array}$ & $\begin{array}{c}1 \\
120\end{array}$ & 110 & $\begin{array}{c}1 \\
100\end{array}$ & $\begin{array}{l}1 \\
90\end{array}$ & $\begin{array}{l}1 \\
80\end{array}$ & $\begin{array}{l}1 \\
70\end{array}$ & & $\begin{array}{l}1 \\
50\end{array}$ & & 30 & $\begin{array}{l}1 \\
20\end{array}$ & $\begin{array}{l}1 \\
10\end{array}$ \\
\hline
\end{tabular}




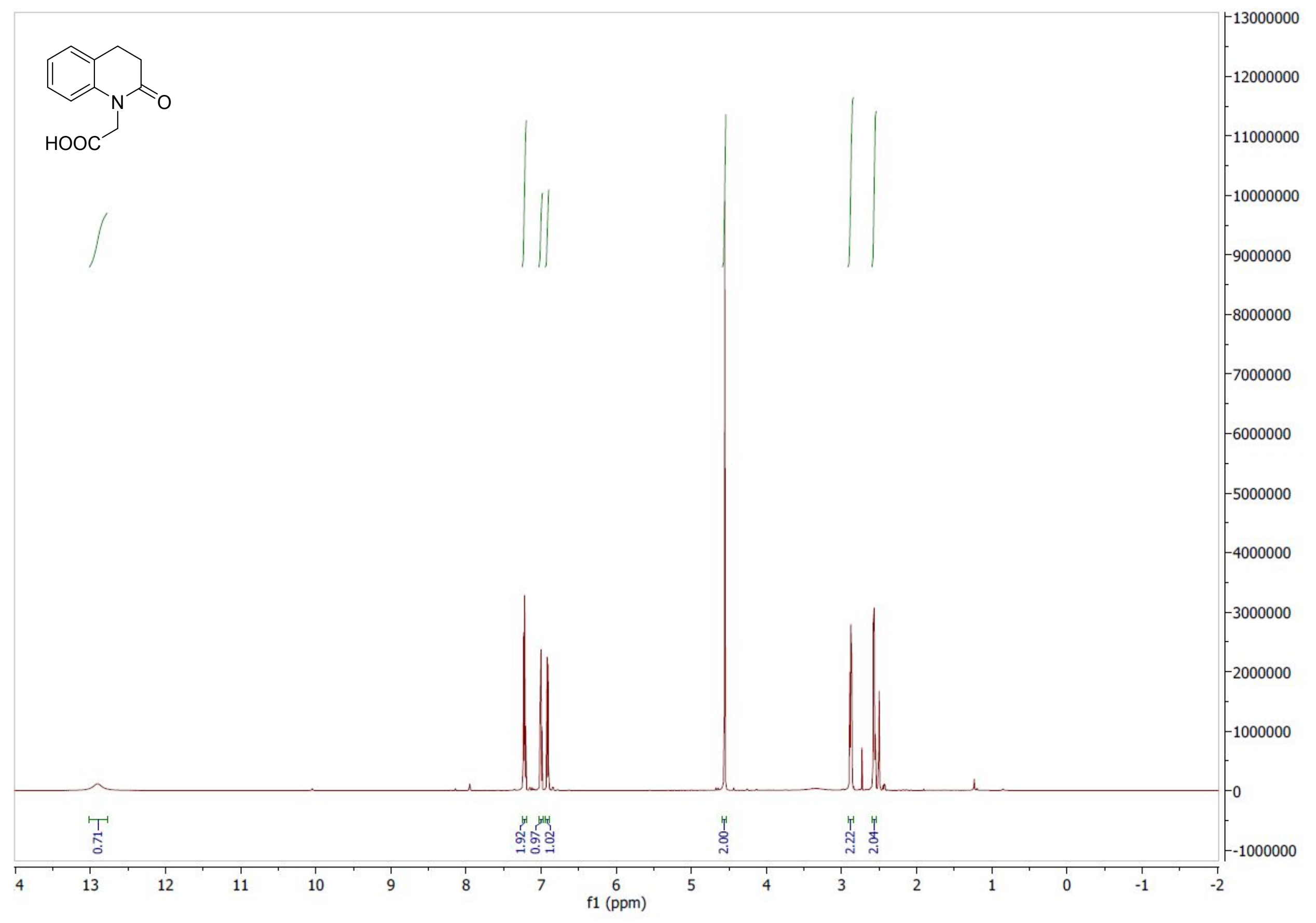




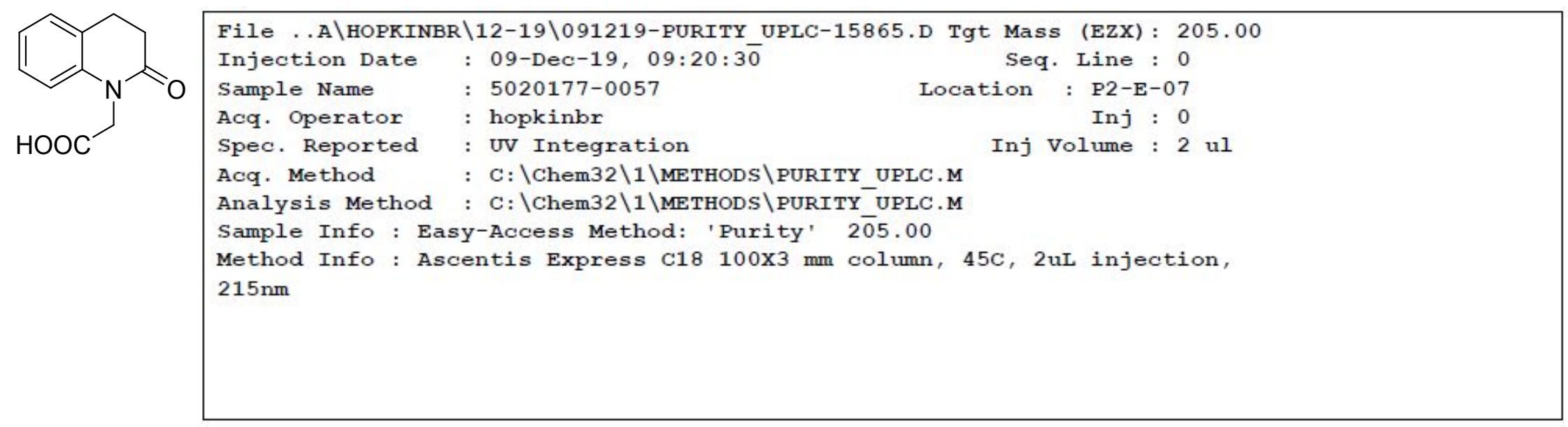

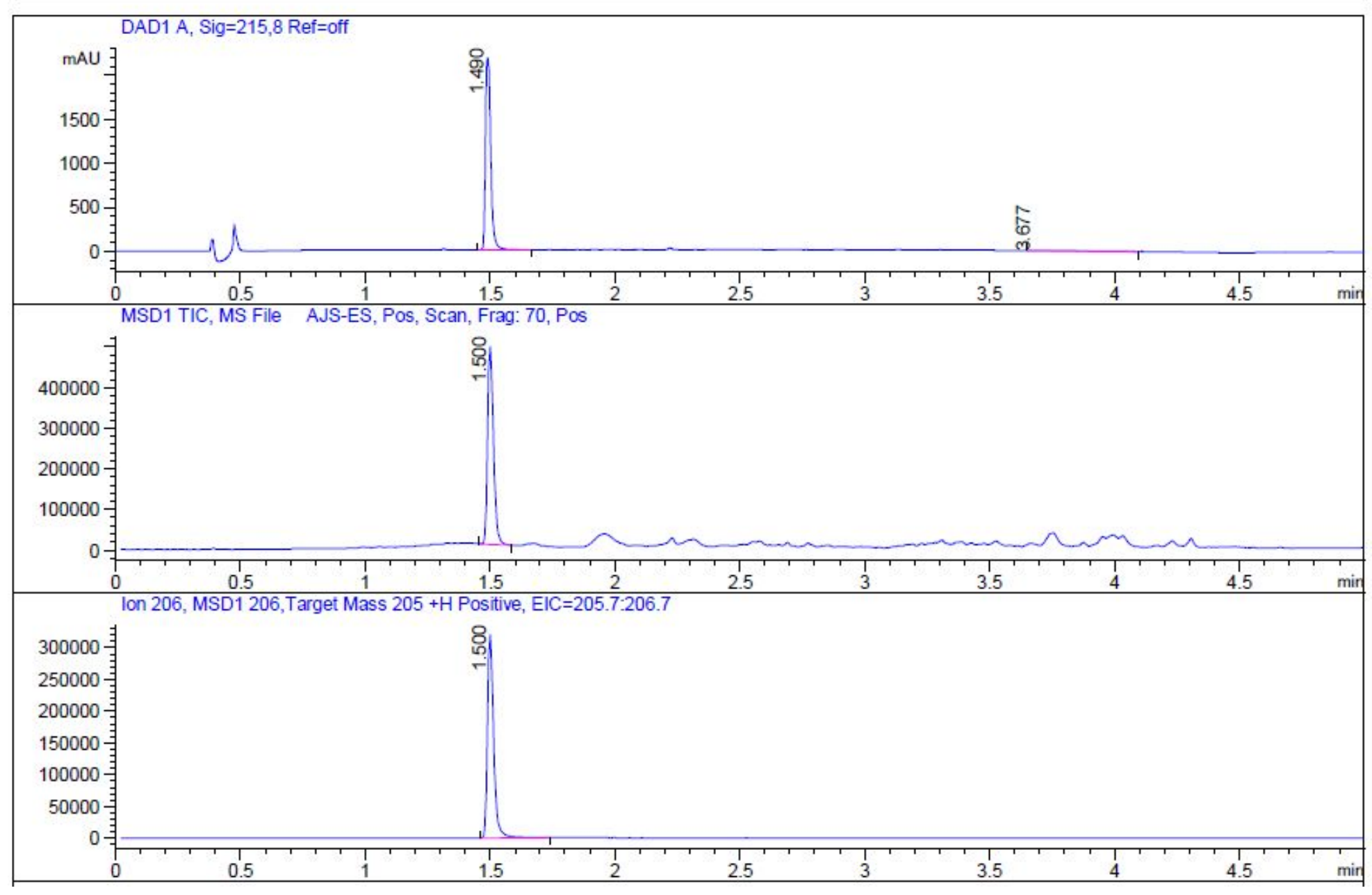




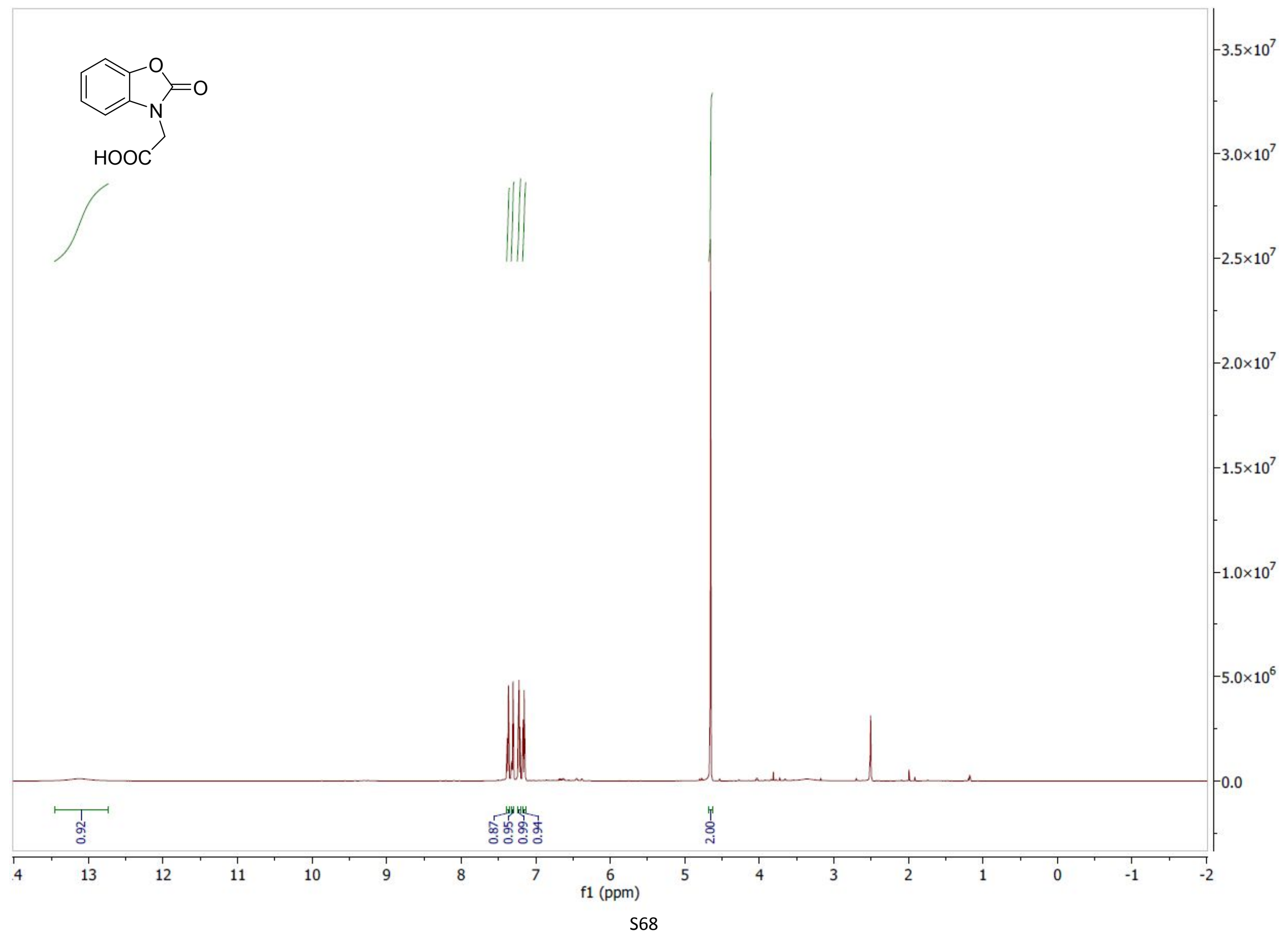


File ..A \HOPKINBR \12-19 \181219-PURITY UPLC-16068.D Tgt Mass (EZX): 193.00

Injection Date : 18 -Dec-19, 12:29:0 $\overline{4}$

Seq. Line : 0

Sample Name : 5020177-0063

Location : P1-E-06

Acq. Operator : hopkinbr

Inj : 0

Spec. Reported : UV Integration

Inj Volume : 2 ul

Acq. Method : C: \Chem32 $\backslash 1 \backslash$ METHODS $\backslash$ PURITY_UPLC.M

Analysis Method : C: \Chem32 $1 \mathbf{1} \backslash$ METHODS $\backslash$ PURITY UPLC.M

Sample Info: Easy-Access Method: 'Purity' $1 \overline{9} 3.00$

Method Info: Ascentis Express c18 $100 \times 3 \mathrm{~mm}$ column, 45C, 2uL injection,

$215 \mathrm{~nm}$

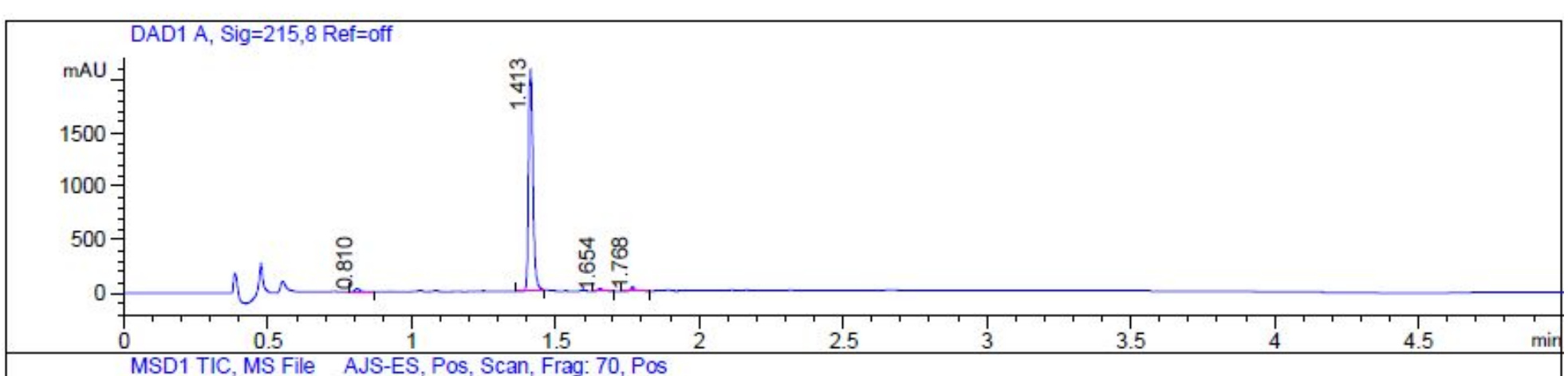

MSD1 TIC, MS File AJS-ES, Pos, Scan, Frag: 70, Pos

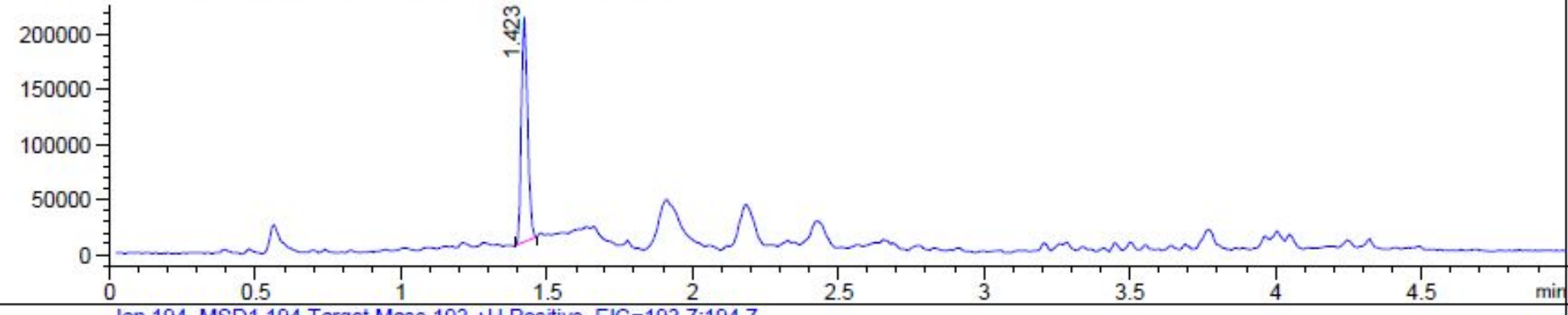

Ion 194, MSD1 194, Target Mass 193 +H Positive, EIC=193.7:194.7

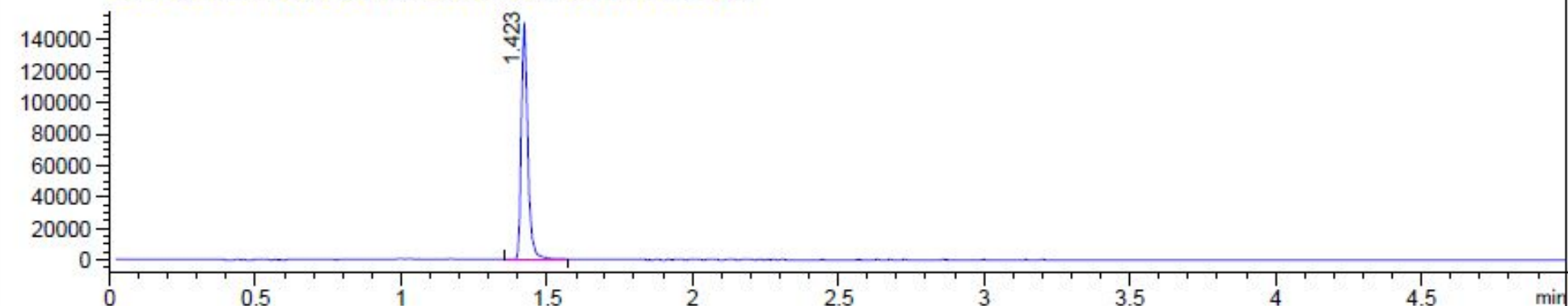

